\title{
Stellar kinematics across the Hubble sequence in the CALIFA survey: general properties and aperture corrections
}

J. Falcón-Barroso ${ }^{1,2}$, M. Lyubenova ${ }^{3,4}$, G. van de Ven $^{4}$, J. Mendez-Abreu ${ }^{5}$, J. A. L. Aguerri ${ }^{1,2}$, B. García-Lorenzo ${ }^{1,2}$, S. Bekeraite ${ }^{6}$, S. F. Sánchez ${ }^{7}$, B. Husemann ${ }^{8}$, R. García-Benito ${ }^{9}$, D. Mast ${ }^{10,11}$, C. J. Walcher ${ }^{6}$, S. Zibetti ${ }^{12}$, J. K. Barrera-Ballesteros ${ }^{13}$, L. Galbany ${ }^{14,15}$, P. Sánchez-Blázquez ${ }^{16,17}$, R. Singh ${ }^{4}$, R. C. E. van den Bosch ${ }^{4}$, V. Wild ${ }^{5}$, L. Zhu ${ }^{4}$, J. Bland-Hawthorn ${ }^{18}$, R. Cid Fernandes ${ }^{19}$, A. de Lorenzo-Cáceres ${ }^{5}$, A. Gallazzi ${ }^{12}$, R. M. González Delgado ${ }^{9}$, R. A. Marino ${ }^{20,21}$, I. Márquez ${ }^{9}$, E. Pérez ${ }^{9}$, I. Pérez ${ }^{22,23}$, M. M. Roth ${ }^{6}$, F. F. Rosales-Ortega ${ }^{24}$, T. Ruiz-Lara ${ }^{22}$, L. Wisotzki ${ }^{6}$, B. Ziegler ${ }^{25}$, and the CALIFA collaboration

${ }^{1}$ Instituto de Astrofísica de Canarias, vía Láctea s/n, 38205 La Laguna, Tenerife, Spain e-mail: jfalcon@iac.es

2 Departamento de Astrofísica, Universidad de La Laguna, 38205 La Laguna, Tenerife, Spain

3 Kapteyn Astronomical Institute, University of Groningen,Postbus 800, 9700 AV Groningen, The Netherlands

${ }^{4}$ Max-Planck-Institut für Astronomie, Königstuhl 17, 69117 Heidelberg, Germany

5 School of Physics and Astronomy, University of St Andrews, SUPA, North Haugh, St Andrews, KY16 9SS, UK

${ }^{6}$ Leibniz-Institut für Astrophysik Potsdam (AIP), An der Sternwarte 16, 14482 Potsdam, Germany

7 Instituto de Astronomía, Universidad Nacional Autónoma de México, Apartado Postal 70-264, México D.F., 04510 México, Mexico

${ }^{8}$ European Southern Observatory, Karl-Schwarzschild-Str. 2, 85748 Garching b. München, Germany

9 Instituto de Astrofísica de Andalucía (IAA/CSIC), Glorieta de la Astronomía s/n Aptdo. 3004, 18080 Granada, Spain

10 Observatorio Astronómico, Laprida 854, X5000BGR, Córdoba, Argentina

11 Consejo de Investigaciones Científicas y Técnicas de la República Argentina, Avda. Rivadavia 1917, C1033AAJ, CABA, Argentina

12 INAF-Osservatorio Astrofisico di Arcetri, Largo Enrico Fermi 5, 50125 Firenze, Italy

13 Space Telescope Science Institute, 3700 San Martin Drive, Baltimore, MD 21218, USA

14 Millennium Institute of Astrophysics, Chile

15 Departamento de Astronomía, Universidad de Chile, Camino El Observatorio 1515, Las Condes, Santiago, Chile

16 Departamento de Física Teórica, Universidad Autónoma de Madrid, 28049 Madrid, Spain

17 Instituto de Astrofísica, Pontificia Universidad Católica de Chile, Av. Vicuña Mackenna, 4860 Santiago, Chile

18 Sydney Institute for Astronomy, School of Physics A28, University of Sydney, NSW 2006, Australia

19 Departamento de Física, Universidade Federal de Santa Catarina, PO Box 476, 88040-900 Florianópolis, SC, Brazil

${ }^{20}$ Departamento de Astrofísica y CC. de la Atmósfera, Universidad Complutense de Madrid, 28040 Madrid, Spain

${ }^{21}$ ETH Zürich, Institute for Astronomy, Wolfgang-Pauli-Str. 27, 8093 Zürich, Switzerland

${ }^{22}$ Departamento de Física Teórica y del Cosmos, University of Granada, Facultad de Ciencias (Edificio Mecenas), 18071 Granada, Spain

23 Instituto Carlos I de Física Teórica y Computacional, Spain

${ }^{24}$ Instituto Nacional de Astrofísica, Óptica y Electrónica, Luis E. Erro 1, 72840 Tonantzintla, Puebla, Mexico

25 University of Vienna, Department of Astrophysics, Türkenschanzstr. 17, 1180 Vienna, Austria

Received 1 April 2016 / Accepted 12 August 2016

\section{ABSTRACT}

We present the stellar kinematic maps of a large sample of galaxies from the integral-field spectroscopic survey CALIFA. The sample comprises 300 galaxies displaying a wide range of morphologies across the Hubble sequence, from ellipticals to late-type spirals. This dataset allows us to homogeneously extract stellar kinematics up to several effective radii. In this paper, we describe the level of completeness of this subset of galaxies with respect to the full CALIFA sample, as well as the virtues and limitations of the kinematic extraction compared to other well-known integral-field surveys. In addition, we provide averaged integrated velocity dispersion radial profiles for different galaxy types, which are particularly useful to apply aperture corrections for single aperture measurements or poorly resolved stellar kinematics of high-redshift sources. The work presented in this paper sets the basis for the study of more general properties of galaxies that will be explored in subsequent papers of the survey.

Key words. galaxies: kinematics and dynamics - galaxies: elliptical and lenticular, cD - galaxies: spiral - galaxies: structure galaxies: evolution - galaxies: formation

\section{Introduction}

The motion of stars within galaxies is a fundamental property set very early on in their life. Ever since the detection of rotation of stars in the Milky Way and nearby systems (e.g., Lindblad 1927; Mayall 1951; Münch \& Münch 1960), the study of stellar motions has been a fruitful avenue to pose important constraints on our knowledge about galaxy formation and evolution. 
The analysis of rotational over random motions in early-type galaxies, for instance, has led to the realization that bright earlytype galaxies are likely triaxial objects supported by orbital anisotropy (e.g., Bertola \& Capaccioli 1975; Illingworth 1977; Binney 1978), rather than rotation.

The coupling of long-slit spectrographs with telescopes 2 to $4 \mathrm{~m}$ in size has provided, over the last three decades, a wealth of spatially resolved observations that has greatly improved our understanding of the overall stellar motion and level of kinematic substructure in external galaxies (e.g., Davies et al. 1983; Bertola et al. 1984; Bender et al. 1994; Fisher 1997; Simien \& Prugniel 1997; Rubin et al. 1999; Vega Beltrán et al. 2001; Aguerri et al. 2003; Falcón-Barroso et al. 2003; Pizzella et al. 2004; van den Bosch et al. 2015).

While the first integral-field units (IFUs) were already in place in the mid-90's (e.g., Bacon et al. 1995), the first serious efforts to measure stellar kinematics on large samples of galaxies using these kinds of instruments did not occur until year 2001. One of the pioneer projects in this respect was the SAURON survey (Bacon et al. 2001; de Zeeuw et al. 2002). With a representative sample of 72 galaxies (24 ellipticals, 24 lenticulars, and 24 early-type spirals, later extended with observations of 18 late-type spirals), this survey has set the reference for stellar kinematic IFU studies (e.g., Emsellem et al. 2004; Falcón-Barroso et al. 2006; Ganda et al. 2006). The discovery of the slow and fast rotator families in early-type galaxies (Emsellem et al. 2007) served as the trigger for a larger project: the ATLAS $^{3 \mathrm{D}}$ survey (Cappellari et al. 2011), in which a volume complete sample of 260 early-type galaxies revisited many kinematic aspects, from the amount of global angular momentum (Emsellem et al. 2011) to a detailed account of kinemetric features (Krajnović et al. 2006, 2011). In parallel, the DiskMass survey mapped, the stellar kinematic properties of nearby latetype spirals with the aid of the PPak IFU (Roth et al. 2005; Kelz et al. 2006).

The CALIFA survey (Sánchez et al. 2012) was born to fill in existing gaps in other IFU surveys and to provide a morphologically unbiased view of the stellar kinematics in galaxies based on a large ( 600 galaxies) and homogeneous integral-field spectroscopic dataset. The main advantage of CALIFA over existing surveys resides in a sample selection that includes all morphological types, as well as a field-of-view (FoV) that extends up to several effective radii $\left(R_{\mathrm{e}}\right)$. While CALIFA is no longer the IFU survey with the largest number of observed objects in the nearby Universe, it still provides the best compromise between spatial coverage $\left(1.8-3.7 R_{\mathrm{e}}\right)$ and sampling $(\sim 1 \mathrm{kpc})$. Currently ongoing IFU surveys are hampered in one way or another by these factors, for example, SAMI covers areas within $1.1-2.9 R_{\mathrm{e}}$ with a spatial sampling $\sim 1.7 \mathrm{kpc}$ (Croom et al. 2012; Bryant et al. 2015), while MaNGA primary sample targets have a spatial sampling of $\sim 3 \mathrm{kpc}$ within $1.5 R_{\mathrm{e}}$ (Bundy et al. 2015). The real revolution in this respect will take place when MUSE at the Very Large Telescope (Bacon et al. 2010) is used in survey mode, as anticipated by the very spectacular stellar kinematic cases presented in the first few years of operations (e.g., Emsellem et al. 2014; Krajnović et al. 2015; Gadotti et al. 2015; Iodice et al. 2015).

The goal of this paper is to present the first stellar kinematic maps extracted from the CALIFA survey, describe all the technical details of the extraction, and provide basic stellar velocity dispersion aperture corrections for elliptical and spiral galaxies. The maps presented here have already been used within the survey to establish the effect of galaxy interactions on the stellar kinematics of galaxies (Barrera-Ballesteros et al. 2014, 2015), constrain the pattern speed of barred galaxies across the Hubble sequence (Aguerri et al. 2015), to present a volume-complete Tully-Fisher relation (Bekeraite et al. 2016a), and the velocity function of galaxies as a benchmark for numerical simulations (Bekeraite et al. 2016b). Forthcoming papers of the survey will make use of this information, for example, to revisit the distribution of global angular momentum in nearby galaxies and determine their dark matter content. Falcón-Barroso et al. (2015) provides a preview of some highlights. For results on the kinematics of the ionized gas in CALIFA, see García-Lorenzo et al. (2015).

The paper is organized as follows. Section 2 describes the sample of 300 galaxies used in our study and how this sample compares with the full CALIFA sample. Section 3 summarizes the instrumental setup employed during the observations. In Sect. 4 we provide details of our kinematic extraction and comparisons with other major IFU surveys. Section 5 explains the limit set by our instrumental setup in the measurement of stellar velocity dispersions. In Sect. 6 we provide velocity dispersion aperture corrections for elliptical and spiral galaxies. Finally, we summarize our work and conclusions in Sect. 7.

\section{The CALIFA sample}

This study is based on observations of 300 galaxies drawn from the CALIFA mother and extended samples ${ }^{1}$, which are part of the photometric catalog of the seventh data release (Abazajian et al. 2009) of the Sloan Digital Sky Survey (SDSS). The main selection criteria in the survey is an angular isophotal diameter $\left(45^{\prime \prime} \leq D_{25} \leq 80^{\prime \prime}\right)$, which is followed by a limited range in redshift, $0.005 \leq z \leq 0.03$. These constraints ensure an efficient use of the PPak IFU and excludes, together with an apparent magnitude cut at $r$-band Petrosian magnitude of 20 mag, the presence of too many dwarf galaxies in our sample. Walcher et al. (2014) provides more details about the sample selection criteria and an in-depth discussion of the effects they introduce in the survey.

The CALIFA sample contains a large number of galaxies with diverse kinematic properties: from slow rotating ellipticals, to disk-dominated fast rotating galaxies, and perturbed interacting systems. This paper is based on the V1200 data (see Sect. 3) available until June 2014. We removed from our original sample of 375 galaxies those cases where the quality of the resulting stellar kinematic maps was not sufficient (e.g., poor spatial sampling due to low-quality data) to guarantee a meaningful analysis. We also selected out those cases whose stellar kinematics appeared highly disturbed by the presence of large nearby companions or had clear indications of being in final stages of a merging process. While this criteria excluded cases like ARP 220 (shown in Fig. 3), it did not remove cases like the Mice galaxies (see Wild et al. 2014, for a detailed CALIFA study of this system), where the interaction has not drastically affected the observed kinematics. Barrera-Ballesteros et al. (2014, 2015) carefully examine the stellar kinematics of merging systems in the CALIFA survey. Our final sample thus consists of 300 galaxies.

In Fig. 1 we show the distribution of CALIFA galaxies presented in this paper as a function of Hubble type, stellar mass, and total absolute magnitude in the $r$-band. Hubble type classification was determined after a careful visual inspection by several members of the team. Stellar masses and

1 Extended sample galaxies, two objects in this study, have CALIFA IDs larger than 1000. See Table B.1 and Sánchez et al. (2016) as well. 


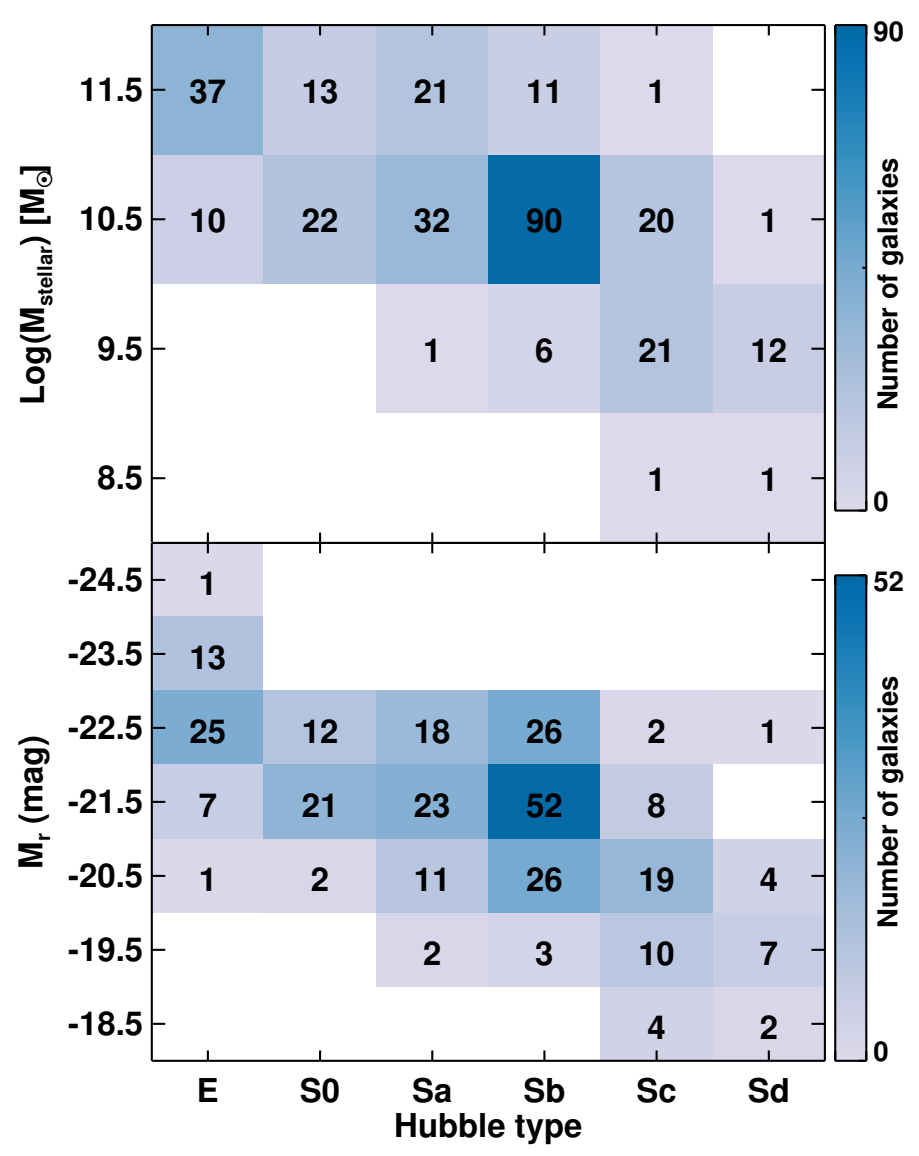

Fig. 1. Distribution of galaxies in the sample of CALIFA galaxies presented in this paper (see Sect. 2) as a function of Hubble type, stellar mass, and absolute magnitude in the $r$-band. For convenience, along with the color bar, we indicate the number of galaxies in each bin.

total absolute magnitudes were derived following the prescriptions described in Walcher et al. (2014). Stellar masses assume a Chabrier initial-mass function (Chabrier 2003). While the number of galaxies represents a major improvement over other integral-field surveys, the selection criteria adopted in the CALIFA survey introduce an important shortcoming: the lack of low-mass, low-luminosity early-type systems and high-mass, high-luminosity late-type galaxies. Another important aspect is that our selection criteria favors edge-on orientations for the lowest mass and fainter systems (i.e., Sd galaxies). The advantage of this selection, however, is that it allows us to volume-correct averaged quantities and thus provide kinematic results that are representative of the general population of galaxies. Table B. 1 contains the basic properties of the subset of galaxies of our study.

We illustrate how representative our subsample is with respect to the mother sample in Fig. 2. The top and middle panels of the figure show the distribution of both the mother sample and our subsample in redshift, isophotal diameter $\left(A_{\text {iso }}\right)$ and petrosian $r$-band magnitude $\left(M_{r, \mathrm{p}}\right)^{2}$. The vertical lines indicate the limits in absolute magnitude in which the CALIFA mother sample is representative. In this space of parameters, the distribution of our subsample is consistent with that shown by the mother sample. Furthermore our galaxies cover all areas sampled by the mother sample. The bottom panel compares the luminosity function of SDSS (Blanton et al. 2003), the CALIFA mother sample, and the

\footnotetext{
2 Total absolute magnitudes are used throughout this paper, except in Fig. 2 where petrosian magnitudes are employed instead for consistency with Walcher et al. (2014).
}

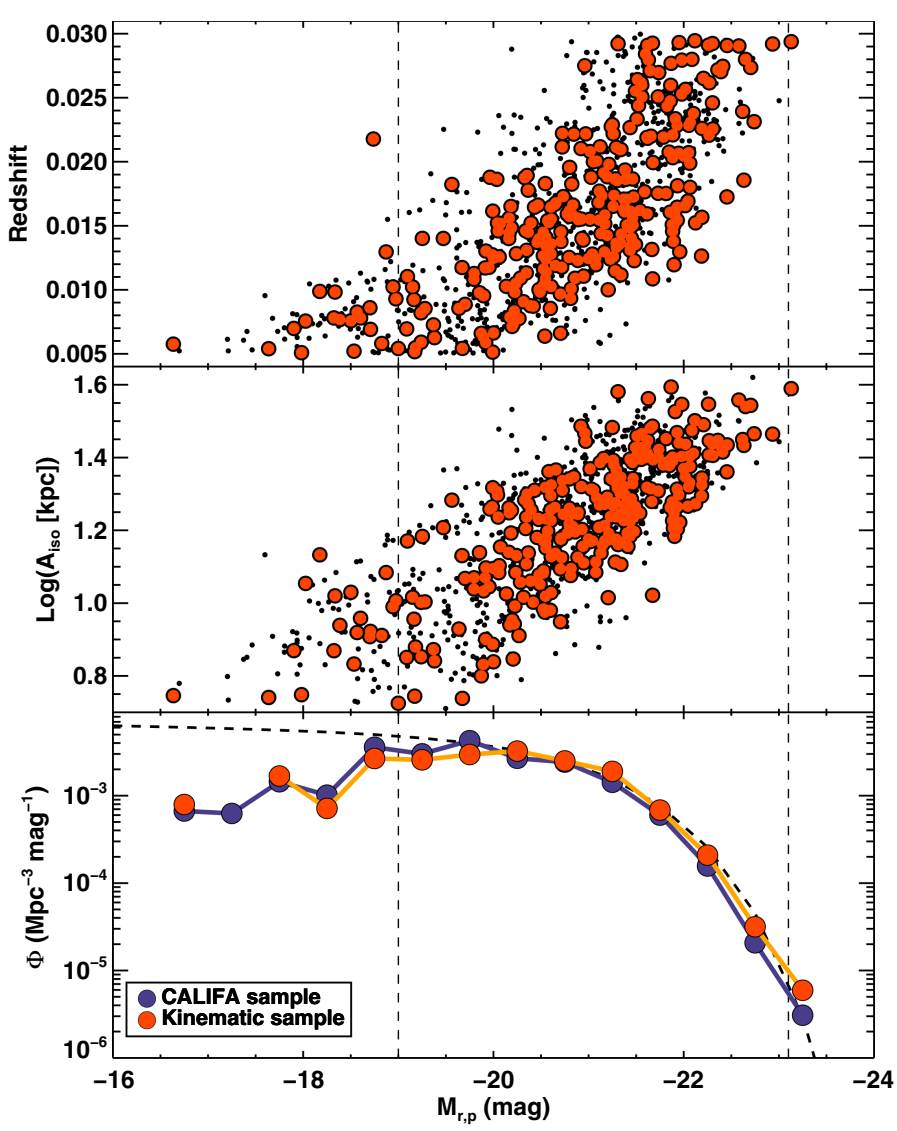

Fig. 2. Top and middle panels: distribution of our sample of 300 galaxies (orange circles) in redshift, isophotal diameter $\left(A_{\text {iso }}\right)$, and absolute $r$-band petrosian magnitude $\left(M_{r, \mathrm{p}}\right)$. For reference, the CALIFA mother sample is shown with black dots. The vertical lines indicate the limits in absolute magnitude in which the CALIFA mother sample is representative. Bottom panel: comparison of the luminosity functions of the SDSS (Blanton et al. 2003, thick dashed line), CALIFA mother sample (dark blue circles), and the kinematic sample presented here (orange circles).

subset of 300 galaxies of the kinematic sample. We have applied a Kolmogorov-Smirnov test to the different parameters and confirm that the kinematic and mother samples are statistically consistent. Therefore the set of 300 galaxies studied in this paper are a good representation of the overall population of galaxies of all morphological types in the nearby Universe, within the luminosity and size constraints imposed by the CALIFA target selection.

\section{Instrumental setup}

The data presented in this paper is part of the CALIFA survey and as such were observed with the PMAS instrument (Roth et al. 2005) in the PPak mode (Verheijen et al. 2004; Kelz et al. 2006), mounted at the $3.5 \mathrm{~m}$ telescope of the Calar Alto observatory. For each galaxy, our observations cover the central $74^{\prime \prime} \times 64^{\prime \prime}$ using a hexagonal fiber bundle. For a detailed description of the observations and data reduction, see the CALIFA presentation article (Sánchez et al. 2012) and the CALIFA Data Release papers 1 and 2 (Husemann et al. 2013; García-Benito et al. 2015). The stellar kinematics presented in this paper is based on data from the $\mathrm{v} 1.4$ data reduction pipeline. Here we give a brief overview of the features of the observational setup that are relevant to our scientific interests. 

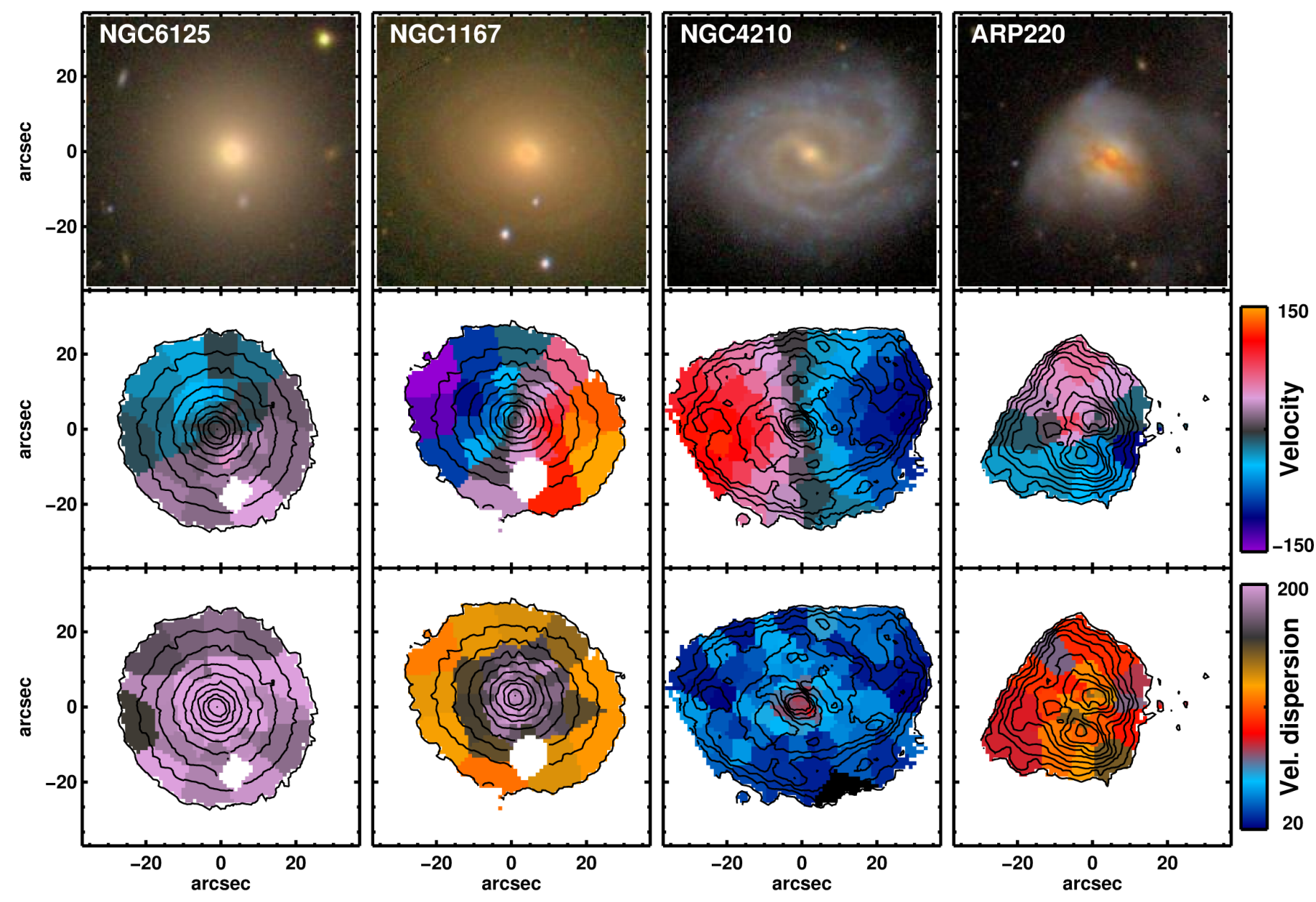

Fig. 3. Examples of line-of-sight stellar kinematic maps from the CALIFA V1200 grating dataset. Top row: color-composite SDSS image of each galaxy. Middle row: stellar velocity maps. Bottom row: stellar velocity dispersion maps. From left to right: NGC 6125, a slow-rotator elliptical in our sample (i.e., low velocity amplitude and overall large velocity dispersion); NGC 1167, an early-type spiral galaxy with large velocity and central velocity dispersion amplitudes; NGC 4210, a disk-dominated galaxy (i.e., high velocity amplitude and overall small velocity dispersion); ARP 220, an interacting system (i.e., with complex stellar velocity and velocity dispersions maps). All maps share the same velocity and velocity dispersion scale and are in units of $\mathrm{km} \mathrm{s}^{-1}$ as indicated in the colorbars. Isophotes (black lines) are constructed from the V1200 CALIFA data cube.

The CALIFA survey is conducted in two instrumental setups: a low resolution mode (V500) with $R \sim 850$ at $\sim 5000 \AA$ and a medium resolution mode (V1200) with $R \sim 1650$ at $\sim 4500 \AA$. The V500 grating covers a broad spectral range (3700-7300 $\mathrm{A})$ and includes a number of absorption and emission features, from the $\mathrm{Ca} \mathrm{H}+\mathrm{K}$ and $[\mathrm{O}$ II] $] \lambda 3727$ to $\mathrm{H} \beta$ and [S II] $\lambda 6731$ lines. The V1200 grating covers a smaller spectral window (3400-4750 Å). After careful evaluation of the spectral resolutions of the two gratings we established a value of $6 \AA\left(F W H M \sim 327 \mathrm{~km} \mathrm{~s}^{-1}\right)$ for the V500 and $2.3 \AA\left(F W H M \sim 169 \mathrm{~km} \mathrm{~s}^{-1}\right)$ for the V1200 gratings, respectively (see Husemann et al. 2013).

\section{Stellar kinematics extraction}

We extracted the stellar kinematics from every galaxy in a uniform way using both instrumental setups, i.e., V500 and V1200. Before accomplishing this, we applied spatial masks to remove spurious effects such as bad pixels, nearby objects, and/or foreground stars. We then logarithmically rebinned the spectra in each data cube to conserve a linear step in velocity space. We trimmed the data to contain only a useful spectral range: 3800 $7000 \AA$ for the V500 and $3750-4550 \AA$ for the V1200 setup. We then selected for future use all spaxels within the isophote level where the average signal-to-noise ratio ${ }^{3}(\mathrm{~S} / \mathrm{N})$ was larger than 3 . This cut ensured the removal of low-quality spaxels, which could introduce undesired systematic effects in our data at low surface brightness regimes. The next step was to spatially bin the data cubes to achieve an approximately constant $\mathrm{S} / \mathrm{N}$ of 20 (per pixel). This value allows us to conserve a good spatial resolution while still being able to reliably estimate the first two moments of the line-of-sight velocity distribution (LOSVD). For this step we used the Voronoi 2D binning method of Cappellari \& Copin (2003). Special care was taken in the $\mathrm{S} / \mathrm{N}$ calculation to account for the correlation in the error spectrum of nearby spaxels (see Husemann et al. 2013, for details).

We measured the stellar kinematics of all galaxies in our sample using the pPXF code of Cappellari \& Emsellem (2004). We used as templates the Indo-US spectral library (Valdes et al. 2004) from which we selected 330 stars that uniformly cover the parameter space in gravity, metallicity, and effective temperature. The careful choice of stellar spectra is crucial to minimize template mismatch effects. We confirmed that, using our subset of $\sim 300$ stars, we could reproduce the same results obtained using the full library. A non-negative linear combination of those

3 We define our $\mathrm{S} / \mathrm{N}$ as the average within the spectral range used in the fitting process. 


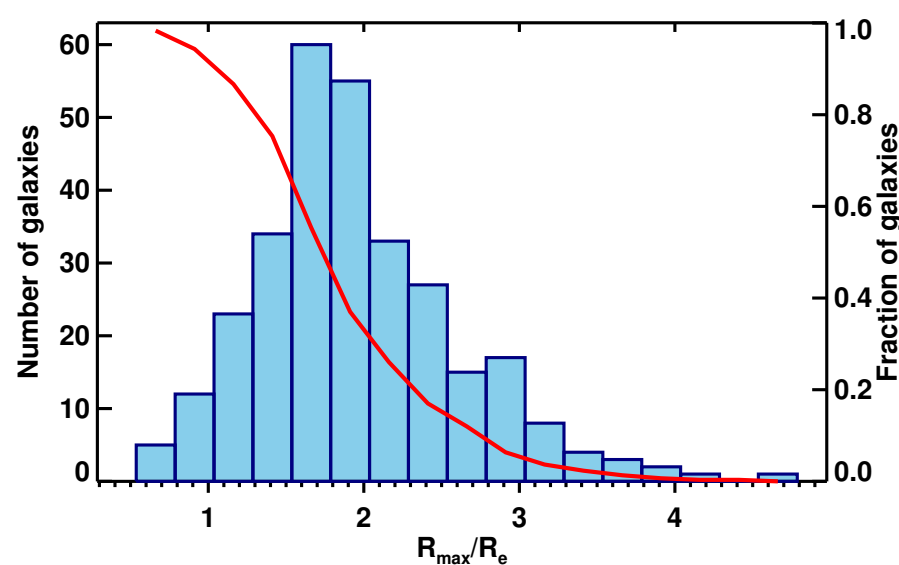

Fig. 4. Distribution of the radial extent of the CALIFA V1200 stellar kinematics. The maximum radius reached in our maps is normalized with the semimajor axis half-light radius $\left(R_{\mathrm{e}}\right)$. The red solid line shows the fraction of galaxies reaching a certain radial extent, as indicated in the right-hand side vertical axis.

stellar templates, convolved with a Gaussian LOSVD, was fitted to the spectrum of each Voronoi bin. The best-fitting parameters were determined by $\chi^{2}$ minimization in pixel space. In the wavelength region covered by CALIFA, there are several emission lines that needed to be masked during the fitting process, for example, [O II], [Ne III], $\mathrm{H} \zeta, \mathrm{H} \epsilon$, [S II], $\mathrm{H} \delta,[\mathrm{Fe}$ II], $\mathrm{H} \gamma$, [O III], $\mathrm{He}$ II, [Ar IV], H $\beta$, [NI], He I, [O I], [N II], and $\mathrm{H} \alpha$. We used a generous band width of $500 \mathrm{~km} \mathrm{~s}^{-1}$ around those lines during the fitting process. This window was enough to mask the emission in all our galaxies. We also masked the regions affected by sky line residuals and the sodium doublet at $\sim 5890 \AA$. Additionally, a low-order additive Legendre polynomial was included in the fit to account for small differences in the continuum shape between the galaxy spectra and the input library. An order 6 polynomial was the minimum that ensured no large-scale wiggles in the residual spectra. In the end, the best-fitting values ( $V$ and $\sigma$ ), and their associated uncertainties, were determined as the bi-weight mean and standard deviations of a set of 100 Monte Carlo realizations of the fitting. As expected, the distribution of best-fitting parameters from the Monte Carlo iterations are wellbehaved and follow a Gaussian distribution. The bi-weight values measured from those distributions agree very well with those obtained from the direct fitting of the original spectra.

In Fig. 3 we show a few representative stellar velocity and velocity dispersion maps obtained with the V1200 grating. The remaining maps are presented in Appendix A. The four examples shown in the figure illustrate the diversity in the kinematics observed in the survey. NGC 6125, is a slow-rotator (e.g., low velocity amplitude and overall large velocity dispersion). NGC 1167 is an early-type spiral galaxy with large velocity and central velocity dispersion amplitudes. NGC 4210 is a disk-dominated galaxy (e.g., high velocity amplitude and overall small velocity dispersion). ARP 220 is an interacting system (e.g., with complex stellar velocity and velocity dispersions maps).

\subsection{Stellar kinematics coverage}

The CALIFA data presented in this paper allow us to produce stellar kinematic maps up to a typical surface brightness level of $\sim 19 \mathrm{mag} \mathrm{arcsec}^{-2}$ (and as faint as $20 \mathrm{mag} \operatorname{arcsec}^{-2}$ ) in $g$-band. We also quantified how far, in terms of $R_{\mathrm{e}}$, our maps extend. This is shown in Fig. 4, where we plot the maximum radius reached

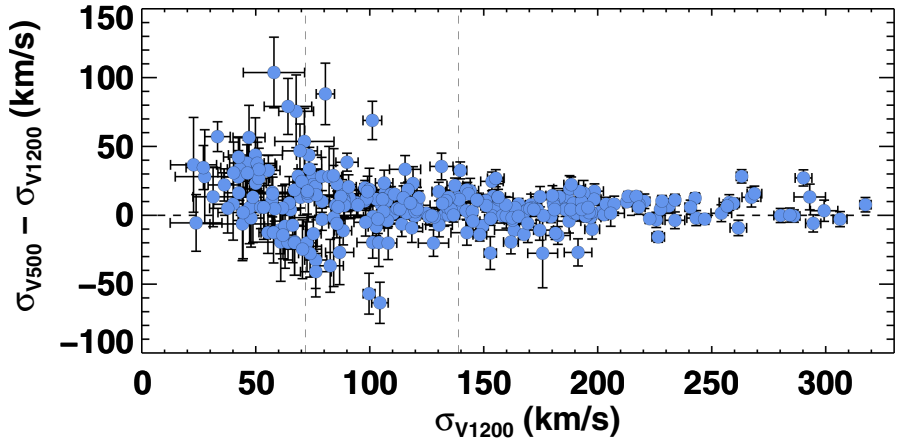

Fig. 5. Comparison of the stellar velocity dispersion from the CALIFA survey for the two instrumental setups: V1200 and V500. The dispersions were computed within an aperture of $3^{\prime \prime}$ diameter (i.e., equivalent to the SDSS fiber aperture). The vertical dashed lines indicate the spectral resolution of the V1200 $\left(\sigma_{\text {instr. }} \sim 72 \mathrm{~km} \mathrm{~s}^{-1}\right)$ and V500 $\left(\sigma_{\text {instr. }} \sim 139 \mathrm{~km} \mathrm{~s}^{-1}\right)$ setups.

by the measurements in our V1200 maps over $R_{\mathrm{e}}$. More than $90 \%$ of the sample covers at least up to $1 R_{\mathrm{e}}$ and $39 \%$ extends beyond $2 R_{\mathrm{e}}$, with $50 \%$ of the galaxies reaching at least $1.8 R_{\mathrm{e}}$. This is a significant improvement over previous IFU surveys (e.g., SAURON, ATLAS ${ }^{3 \mathrm{D}}$ ), which aimed to probe different properties up to $1 R_{\mathrm{e}}$. The strength of those surveys, however, resides in the study of nearby systems at a higher spatial resolution, which allows them to detect small-scale inner kinematic subcomponents (e.g., McDermid et al. 2006; Krajnović et al. 2011).

\subsection{Comparison between V500 and V1200 kinematics}

The two instrumental setups used for the CALIFA survey give us the interesting opportunity of measuring the stellar kinematics of galaxies from independent datasets. As described in Sect. 3, one of these setups (V500) offers a much lower spectral resolution than the other, which turns out to be not enough to measure the lowest velocity dispersions present in our sample. This issue is clearly shown in Fig. 5, which presents the difference in velocity dispersion for each setup, measured within a $3^{\prime \prime}$ diameter aperture centered in each galaxy. In this panel systematic differences appear at dispersion values below $\sim 100 \mathrm{~km} \mathrm{~s}^{-1}$. We also compared the line-of-sight velocities from each setup (not shown here) and, as expected, found that they are well within the uncertainties of our measurements. Given this limitation, from now on we only report about results coming from the V1200 grating.

\subsection{Comparison with other surveys}

As an additional test to check the reliability and accuracy of our kinematic extraction, we compared our central velocity dispersion values with those provided by the SDSS DR7 survey (Abazajian et al. 2009) for those galaxies in our sample with SDSS spectroscopy available. We mimicked the SDSS aperture and extracted our velocity dispersions within a $3^{\prime \prime}$ diameter aperture centered in each galaxy. The result of this comparison is presented in Fig. 6. The agreement between the two sets of measurements is good in general showing only a small systematic offset of $\sim 6 \mathrm{~km} \mathrm{~s}^{-1}$, which is likely due to differences in the extraction method, set of templates, point-spread function (PSF)/seeing effects, and inaccuracies in the determination of the spectral resolution of both the data and templates. Similar levels of discrepancy and systematic differences have been identified in the 


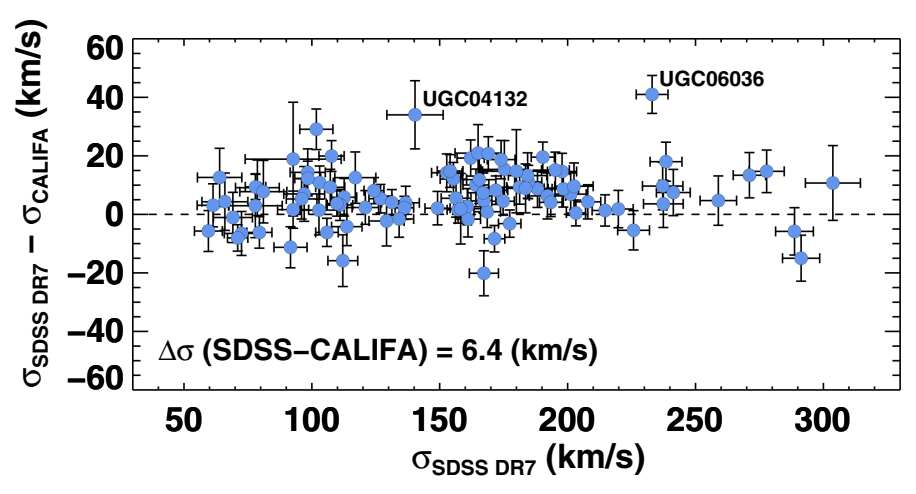

Fig. 6. Comparison of the stellar velocity dispersion from the CALIFA survey with the officially released SDSS DR7 measurements (Abazajian et al. 2009). The dispersions were computed within an aperture of $3^{\prime \prime}$ diameter (i.e., equivalent to the SDSS fiber aperture).

past with SDSS DR7 measurements, even using the same SDSS dataset (see Fig. 6 in Oh et al. 2011).

An even more stringent test is the direct comparison of our stellar velocity dispersion maps to those of other surveys. We found up to six objects in common with one of the reference IFU surveys today, which is ATLAS ${ }^{3 \mathrm{D}}$. We focus our test on the velocity dispersion maps, as the velocity maps (not shown here) are in good agreement. The results of this comparison are presented in Fig. 7. By construction, all the ATLAS ${ }^{3 \mathrm{D}}$ galaxies are early-type systems, which are predominantly red objects with fairly high central velocity dispersions. The figure shows a colorcomposite SDSS image of each galaxy in common, as well as the dispersion maps of both surveys and radial velocity dispersion profiles (extracted in circular annuli). The overall agreement between the two surveys is very good, despite differences in $\mathrm{S} / \mathrm{N}$ thresholds applied in each survey. While the ATLAS ${ }^{3 \mathrm{D}}$ data was Voronoi binned to a $\mathrm{S} / \mathrm{N}$ of 40 , we deemed it necessary to adopt a threshold $\mathrm{S} / \mathrm{N}$ of 20 to find a good balance between spatial resolution (i.e., Voronoi bin sizes) and spatial coverage. The bigger footprint of the PPak IFU allows us to reach well beyond $1 R_{\mathrm{e}}$ for most of the sample (see Fig. 2), which is a significant improvement over ATLAS ${ }^{3 \mathrm{D}}$. The large bins are also responsible for the smoother trends observed in the CALIFA radial profiles.

The only major difference between the two datasets, however, is on the central dispersion values. In general, ATLAS ${ }^{3 \mathrm{D}}$ values are larger. There are two main reasons that could explain this behavior. The PPak IFU is a fiber bundle made of $2.7^{\prime \prime}$ wide fibers, as opposed to ATLAS ${ }^{3 \mathrm{D}}$ with $\sim 1^{\prime \prime}$ lenslets. While our dithering strategy during observations (see Sánchez et al. 2012 , for details) allowed us to resample our final data cube to $1^{\prime \prime}$ per spaxel, the original fiber size could result in lower velocity dispersion values due to beam smearing. We tested this scenario by comparing the central ATLAS ${ }^{3 \mathrm{D}}$ values with those obtained by collapsing the ATLAS ${ }^{3 \mathrm{D}}$ spectra within a $3^{\prime \prime}$ aperture (similar to a CALIFA fiber). Our results show that velocity dispersion values can decrease by up to $15 \%$. This effect can therefore explain part of the discrepancy between the two surveys.

In addition, the effective PSF measured for the CALIFA survey (García-Benito et al. 2015) could also affect these values. While reported seeing conditions between the two surveys appear similar, if the CALIFA PSF was worse than the ATLAS ${ }^{3 D}$ PSF, this could also explain part of the decrease in the central velocity dispersion. Based on some simulations carried out in the context of another CALIFA paper (Méndez-Abreu et al. 2016), we estimated that the PSF can account for up to 5\% difference in the observed values. On top of that, the level of Voronoi binning could play a similar role, although this seems unlikely in our case as the central spaxels remain mostly unbinned.

\section{Reliability of velocity dispersion measurements below the instrumental resolution}

An important aspect to consider when extracting stellar kinematics of galaxies is to understand the limiting velocity dispersions one can reach given the spectral resolution provided by the instrument used. The safest option is to use an instrumental setup where the spectral resolution is better than the expected values. Under certain circumstances, however, it is possible to push this limit and measure velocity dispersions below the nominal threshold imposed by the instrument. As shown in Ryś et al. (2013), but see also González (1993) and Pedraz et al. (2002), a combination of high signal-to-noise and spectral sampling of the line spread function (e.g., Koleva et al. 2009) makes it possible to overcome, to some extent, this limitation.

\subsection{Comparison between DiskMass and CALIFA datasets}

The nominal spectral dispersion of the CALIFA V1200 data is $\sigma_{\text {intr }} \approx 72 \mathrm{~km} \mathrm{~s}^{-1}$. We determined the velocity dispersion limit of our data using as a reference three galaxies in the DiskMass survey (DMS; Bershady et al. 2010). This dataset was designed to measure velocity dispersions in face-on, disk galaxies and the spectral resolution of the instrument $(F W H M=$ $0.69 \AA)$ was chosen to safely reach values around $\sim 17 \mathrm{~km} \mathrm{~s}^{-1}$ (Martinsson et al. 2013). The PPak was custom built for the DMS and subsequently employed in the CALIFA survey, which can help suppress systematic effects inherent in the analysis. The DMS team has kindly provided their data for three galaxies. One was already in common with the CALIFA survey (NGC 234). We observed two more, specifically for these tests, in 21-23 February 2014 (UGC 4256, UGC 4458), using the same V1200 instrumental configuration of the main survey.

Before carrying out our tests, and to account for potential systematic effects, we checked that neither the method (crosscorrelation technique versus pPXF) used to measure the stellar kinematics had a strong impact on the resulting velocity dispersions. Our own extraction, using pPXF, of velocity dispersions from DMS data provided fully consistent results. The choice of templates, whether a single star (as the DMS team used) or a full stellar library (like in our case), did not cause any systematic difference in this particular exercise. Therefore template mismatch is not an issue in these tests. The successful comparison of the two methods using the same data was also reported by Westfall et al. (2011).

In addition to the difference in spectral resolution and template mismatch, there are some further differences with respect to the DMS team analysis that can cause systematic effects in the velocity dispersion values. Spatial binning is desirable in general to reach a threshold $\mathrm{S} / \mathrm{N}$, but it can also have the negative effect of artificially broadening the line-of-sight velocity distribution. This is more acute in the outer regions of galaxies, where the $\mathrm{S} / \mathrm{N}$ drops quickly and the combination of a larger number of spectra is required. Despite this drawback, we chose to Voronoi bin the data to ensure a minimum quality of the spectra used to derive the velocity dispersion. The DMS team preferred to extract their values on single spaxels of $\sim 3^{\prime \prime}$ diameter, and remove values with uncertainties larger than $8 \mathrm{~km} \mathrm{~s}^{-1}$ (see Sect. 7.3.2 of Martinsson et al. 2013). 
J. Falcón-Barroso et al.: Stellar kinematics across the Hubble sequence
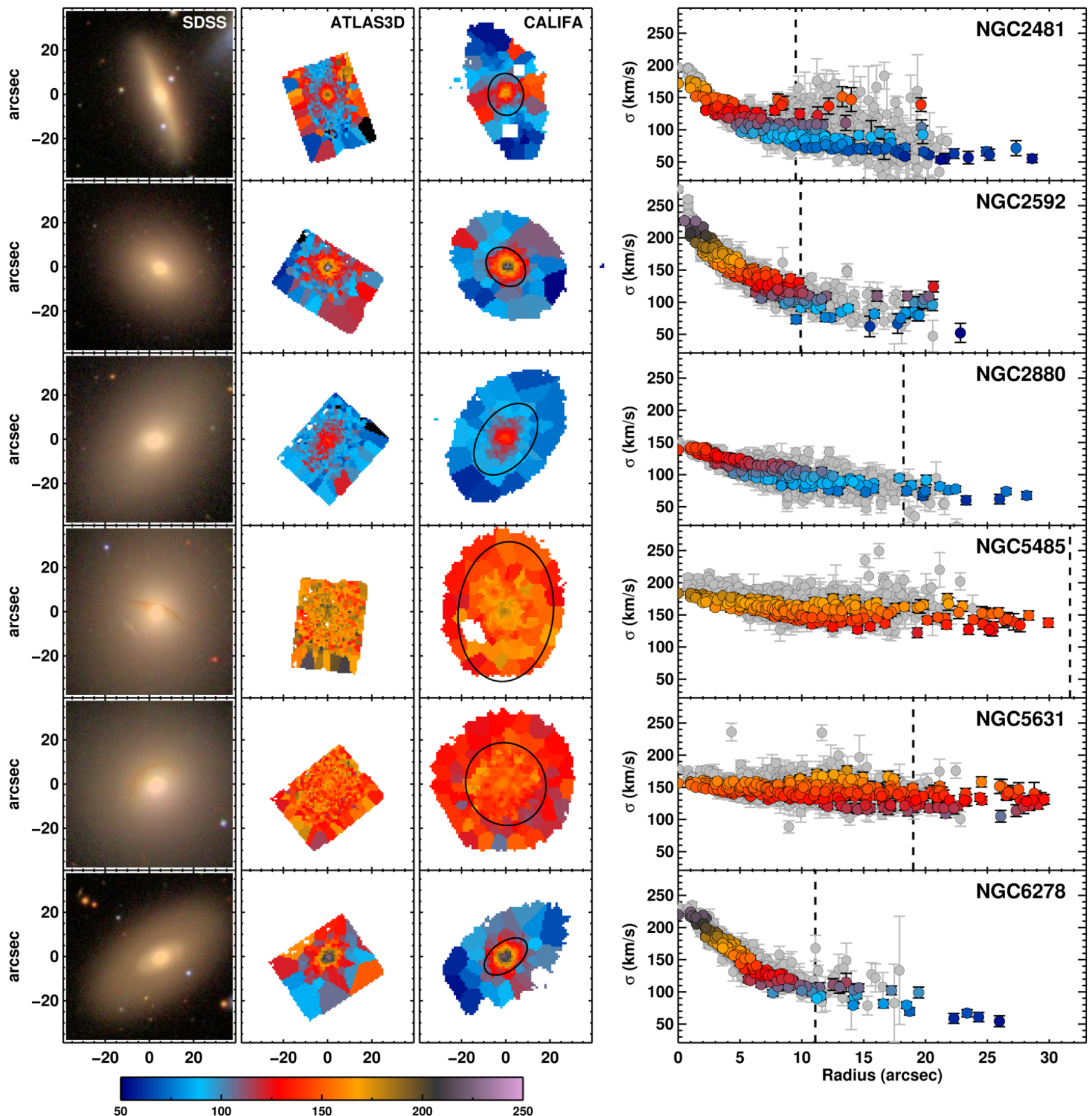

Fig. 7. Comparison of the stellar velocity dispersion maps and radial profiles from the CALIFA and ATLAS ${ }^{3 \mathrm{D}}$ surveys. Each row shows (from left to right): a color-composite SDSS image of the galaxy, the ATLAS ${ }^{3 \mathrm{D}}$ velocity dispersion map, the CALIFA velocity dispersion map, and the radial velocity dispersion profile (extracted in circular apertures). ATLAS ${ }^{3 \mathrm{D}}$ measurements are in gray while the CALIFA measurements are in color following the same color scheme of the maps (also indicated with the colorbar below). The black ellipse in the maps indicates one effective radius in those galaxies. This is also indicated in the radial profile panels with a dashed vertical line. Empty (i.e., white) regions within some of the CALIFA maps are areas masked during our kinematic extraction. All velocity dispersion measurements are expressed in $\mathrm{km} \mathrm{s}^{-1}$.

Another important issue is the wavelength range used to derive the velocity dispersion. The DMS values rely on measurements in the short spectral range between 4980-5370 ̊. Our CALIFA values are based on fits between $3750-4550 \AA$. While a longer baseline is in principle preferred, different spectral features may have slightly distinct intrinsic broadening (at the spectral resolutions we are considering here). We believe this may be the case in the CALIFA spectral range with the $\mathrm{Ca} \mathrm{H}+\mathrm{K}$ lines. We attempted to derive our stellar kinematics ignoring those lines, but results were noisier and uncertainties larger as the fits rely on a few weak spectral features, for example, Fe ( $\lambda 4383 \AA), \mathrm{H} \gamma$, and $\mathrm{H} \delta$. As shown in Kobulnicky \& Gebhardt (2000), the $\mathrm{Ca} \mathrm{H}+\mathrm{K}$ lines are reliable features to obtain stellar kinematics in all kinds of galaxies, although their results 

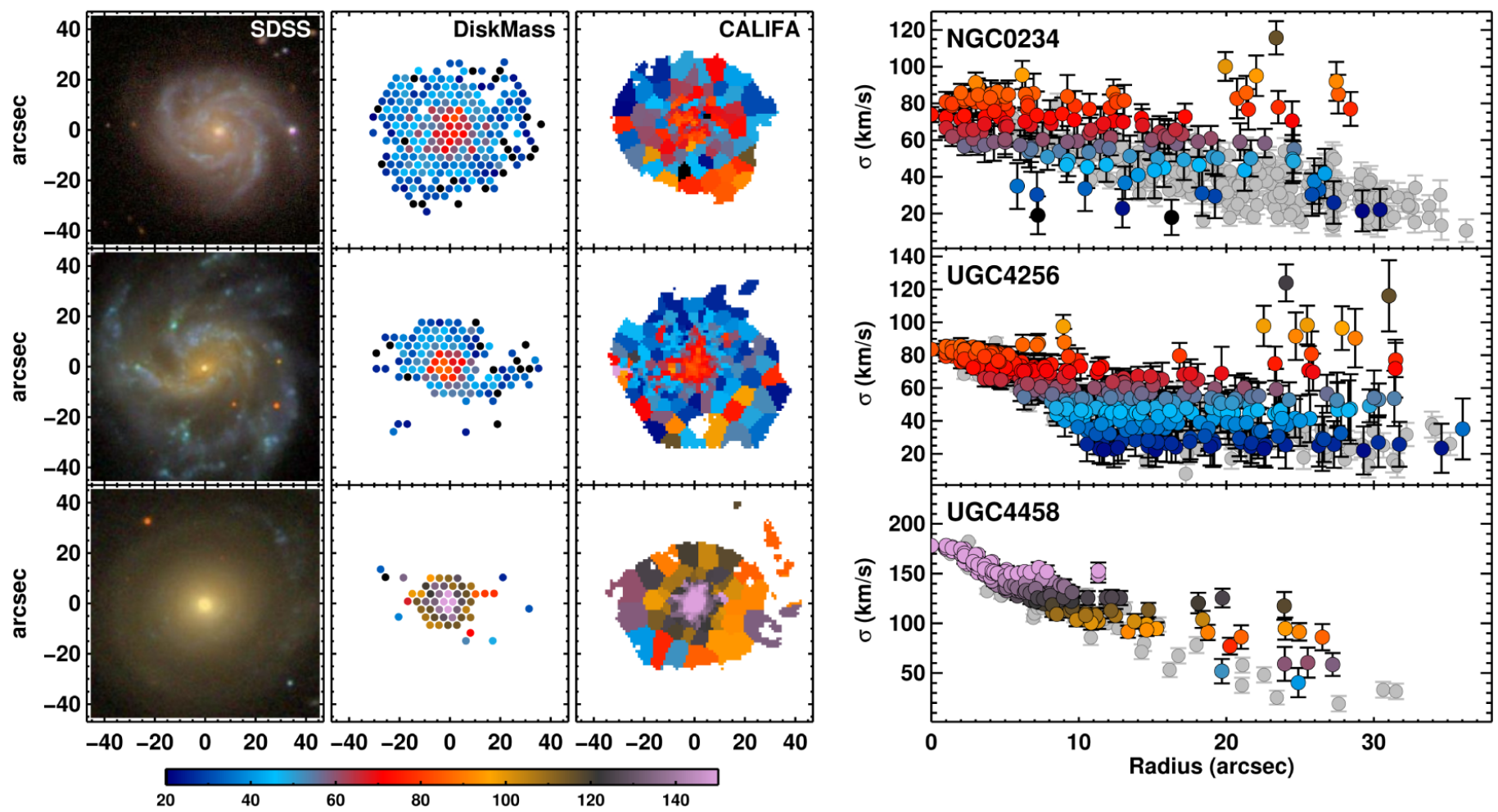

Fig. 8. Comparison of the stellar velocity dispersion maps and radial profiles from the CALIFA and DiskMass surveys. Each row shows (from left to right): a color-composite SDSS image of the galaxy, the DiskMass velocity dispersion map (see Sect. 5 for details), the CALIFA velocity dispersion map, and the radial velocity dispersion profile (extracted in circular annuli). DiskMass measurements are in gray while the CALIFA measurements are in color following the same color scheme of the maps (also indicated with the colorbar below). Empty (i.e., white) regions within some of the CALIFA maps are areas masked during our kinematic extraction. All velocity dispersion measurements are expressed in $\mathrm{km} \mathrm{s}^{-1}$.

appeared to be more uncertain for late-type systems. This may be the culprit of some of the differences we see with the DiskMass survey (see below). The detailed characterization of all these effects is a complex task, and even if we could measure the systematic deviations introduced by each effect, it is not totally obvious they would affect different kinds of galaxies in the same manner (e.g., emission-free early-type galaxies versus highly starforming spiral disks).

Figure 8 shows the comparison of our own CALIFA data with the stellar velocity dispersions measured by the DMS team on the three galaxies in common. As in Fig. 7, we plot a SDSS color image of each galaxy, velocity dispersion maps of the two surveys, and also radial profiles (extracted in circular annuli) for a more direct comparison. When displaying the maps and radial profiles, we plot the individual spaxel measurements provided by the DMS team and our Voronoi binned values. The agreement between the two datasets is good overall. We do see discrepancies in some measurements (most noticeable in NGC 234 and UGC 4256). It appears that some of our CALIFA measurements are much larger than those reported by the DMS team at a given radius. We explored the reasons for these discrepant values and concluded that they occur in low surface brightness regions $\left(\mu_{\mathrm{B}} \geq 22 \mathrm{mag} \operatorname{arcsec}{ }^{2}\right)$ that are often affected by dust or unmasked foreground stars. They often have $\mathrm{S} / \mathrm{N}$ slightly below the nominal $S / N=20$ threshold, which is permitted by the Voronoi binning routine within some tolerance (see Cappellari \& Copin 2003). These values are also naturally associated with large Voronoi bins, which can also artificially help to increase the broadening. However, the pPXF fits in those regions are not particularly worse than in other areas with similar level of binning, $\mathrm{S} / \mathrm{N}$, or surface brightness levels. Given that there might be some physical insight as to why those values are high (e.g., dust obscuration, multiple kinematic components, and kinematic flaring in the outer parts of galaxies), we prefer to keep them in our data release and let the user, based on diagnostic parameters we provide, decide whether to include or exclude them depending on their science case. This effect is not evident in the DMS values owing to the partial field-of-view coverage of their data.

\subsection{Limiting velocity dispersion and relative uncertainties}

In order to establish the lowest reliable velocity dispersion we can measure, we directly compared the DMS and ATLAS3D values to our CALIFA measurements. This is shown in the top panel of Fig. 9, where we present the ratio of the CALIFA over the DMS and ATLAS3D dispersion values as a function of the DMS or ATLAS3D measurements. This exercise determines at which velocity dispersion values our CALIFA measurements depart systematically from the one-to-one relation. For a fair comparison, we used the Voronoi values of our CALIFA maps at the locations of DMS measurements. This is a better approach than interpolating our maps at those locations, which may produce artifacts. The drawback of this approach is that there is some instrinsic scatter produced by the sampling of our points in locations that could be far from the Voronoi centroids in our data. It is also sensitive to the different levels of scatter of the data points in the surveys (e.g., the scatter of ATLAS3D points is larger than CALIFA, see Fig. 7). While the number of points is not too large for the DMS survey ( 360 measurements), it is enough to compute some statistics. Besides the individual datapoints, we indicate the limiting $16 \%$ and $84 \%$ percentiles of the distribution with dotted lines. The median of the distribution is marked with a solid line. The panel shows that velocity dispersion values 

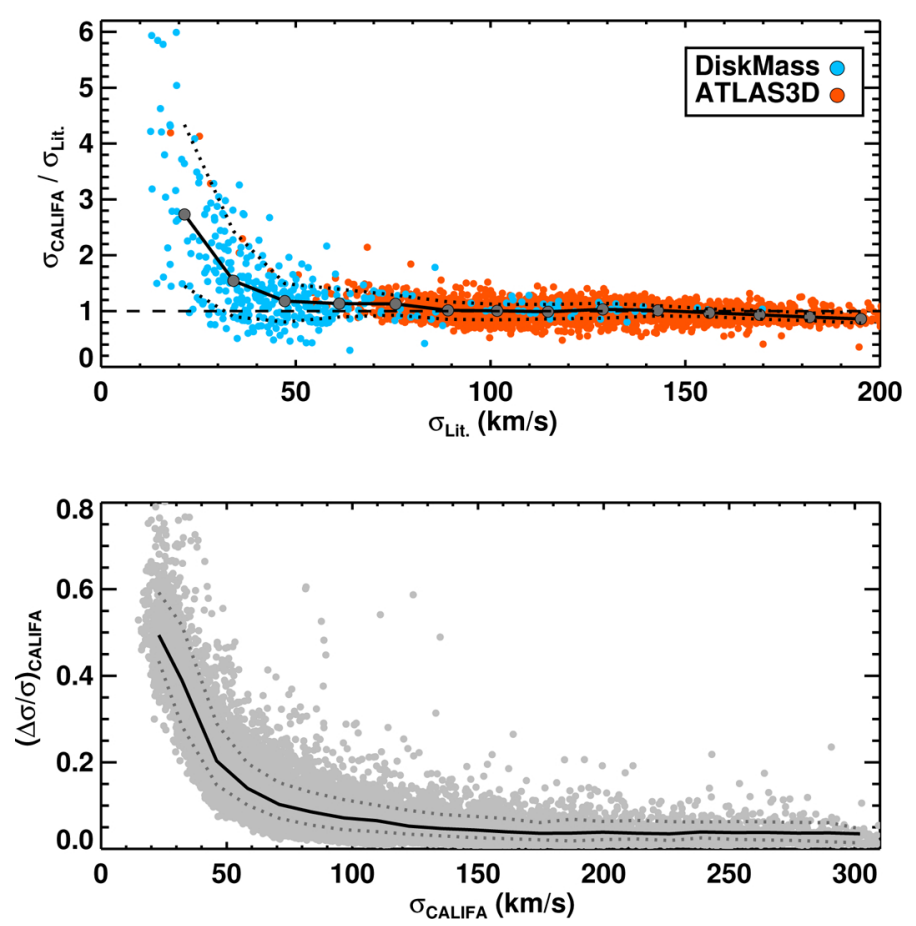

Fig. 9. Characterization of the biases and relative uncertainties in the velocity dispersions of the CALIFA survey. Top panel: ratio between the CALIFA and DMS/ATLAS3D measurements at the locations of the DMS/ATLAS3D values (see Sect. 5.2 for details). Bottom panel: relative uncertainties in the velocity dispersion values of the CALIFA survey using the Voronoi values and uncertainties for all the galaxies presented here. In both panels, the area delimited by the dotted lines indicates the $16 \%$ and $84 \%$ percentiles of the distribution of gray points. The solid lines and gray points indicate the median of the distributions.

are consistent within the uncertainties down to $\sim 40 \mathrm{~km} \mathrm{~s}^{-1}$. Below that point, CALIFA measurements are systematically larger up to a factor $\sim 3$ on average for $\sigma$ values around $20 \mathrm{~km} \mathrm{~s}^{-1}$. On the high velocity dispersion end, values converge asymptotically to unity, as expected, except for the most massive systems where we suffer the PSF and beam smearing effects discussed in Sect. 4.3 for ATLAS3D.

In addition to the potential bias in our measurements, it is also interesting to determine the relative uncertainties of our measurements at different velocity dispersion regimes. This is presented in the bottom panel of Fig. 9. We produced this figure using all the individual Voronoi bin measurements and uncertainties for the 300 CALIFA galaxies presented here. The shaded region and lines as in the top panel. The figure shows that uncertainties are rather small around $5 \%$ for $\sigma \geq 150 \mathrm{~km} \mathrm{~s}^{-1}$. Below that value, relative uncertainties increase up to $50 \%$ for velocity dispersions $\sigma \sim 20 \mathrm{~km} \mathrm{~s}^{-1}$. The median uncertainty at $\sigma \sim 40 \mathrm{~km} \mathrm{~s}^{-1}$, where our measurements start deviating systematically from the DMS values, is $\sim 20 \%$.

\section{Aperture profiles}

The large number of galaxies across the Hubble sequence in our study allows us to estimate velocity dispersion aperture corrections for different groups of galaxies. These corrections are useful to homogenize dispersion values measured with fiber-fed spectrographs (e.g., SDSS) for galaxies at different distances, and they are particularly important for high-redshift studies.

We studied the behavior of the integrated velocity dispersion profiles of our galaxies, extracted in elliptical apertures with a

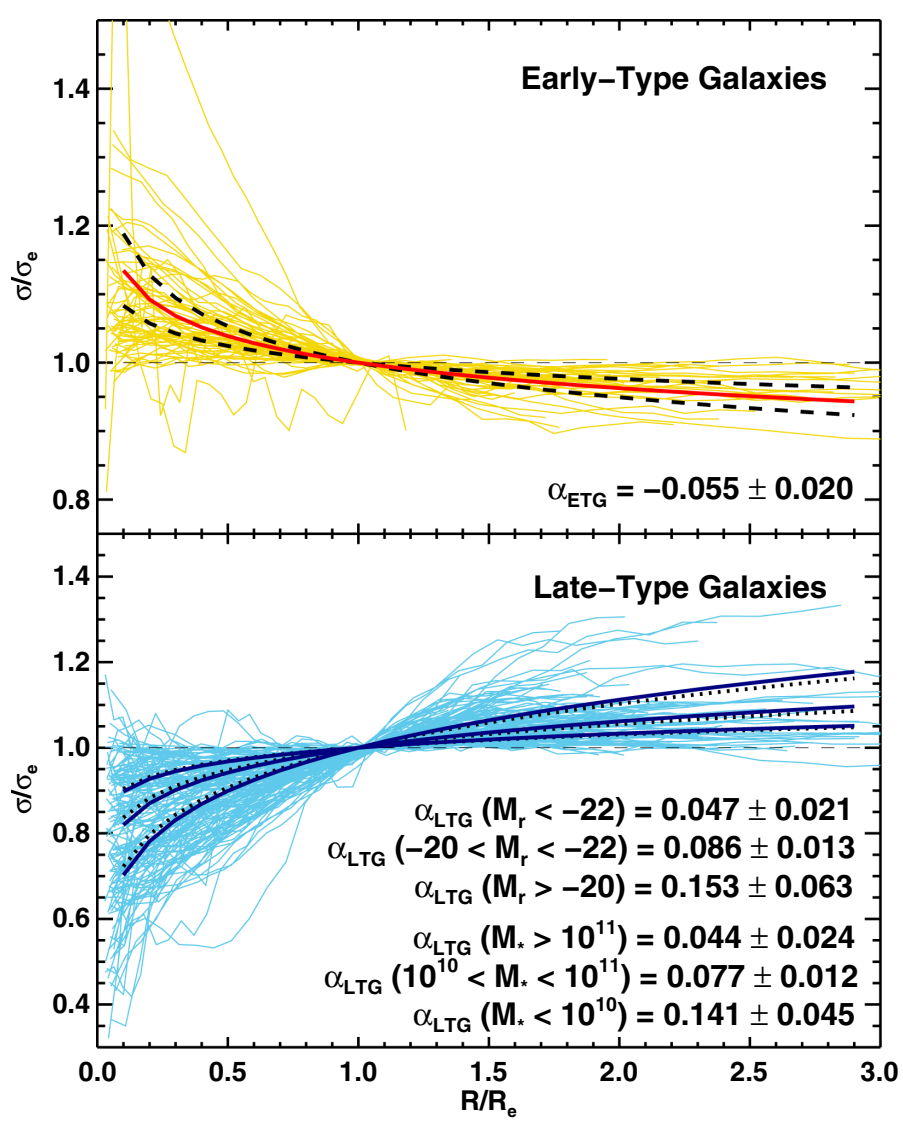

Fig. 10. Stellar velocity dispersion profiles integrated within elliptical apertures with increasing semimajor radius. The profiles are normalized by the effective velocity dispersion $\left(\sigma_{\mathrm{e}}\right.$ within the effective radius $\left.\left(R_{\mathrm{e}}\right)\right)$. The galaxies were divided depending on the shape of their profile: (top panel) declining galaxies and (bottom panel) steadily increasing galaxies, which naturally correspond to early-type and late-type galaxies, respectively. For early-type galaxies, the red line is the average fit taking volume corrections into account. Dashed lines indicate the uncertainty of the fit. For late-type galaxies, dotted lines indicate average fits for different intervals of stellar mass, while solid lines indicate average fits for different intervals of absolute magnitude. For clarity, we did not include the lines with uncertainties in these cases. Averages for late types also take volume corrections into account.

fixed position angle and ellipticity (as indicated in Table B.1). We chose elliptical rather than circular apertures to properly account for inclination effects. We used the position angle and ellipticity measured in the outer parts of the galaxy (as listed in Table B.1). While this choice ignores potential radial variations in these two parameters (e.g., due to bars), the velocity dispersion maps do not appear to be clearly influenced by those photometric deviations. This is true in particular for barred galaxies, as shown in Seidel et al. (2015) or Gadotti et al. (2015).

We found three types of radial profiles: (Class 1) those that decrease steadily, (Class 2) those decreasing up to a certain radius and then increasing again, and (Class 3) those that increase steadily with radius. We analyzed the type of galaxies conforming each class and found that class 1 objects are typically early types (e.g., ellipticals, lenticulars and Sa galaxies). Class 2 is made of a rather small set of objects $(\sim 20)$ that are mostly earlytype galaxies too. They show fairly high dispersions in the center but also reasonably high rotation in the outer parts, which drive the increase of integrated velocity dispersion at large radii. This effect is even more pronounced in class 3 objects, that are predominantly late-type systems (e.g., Sb, Sc, and Sd galaxies). 
Given the small number of objects in class 2, we only provide aperture corrections for the other two groups (class 1 and 3). We followed previous works in the literature and fit the individual profiles of each galaxy in these two classes using a power-law function in the form

$\left(\frac{\sigma}{\sigma_{\mathrm{e}}}\right)=\left(\frac{R}{R_{\mathrm{e}}}\right)^{\alpha}$,

where the effective radius $\left(R_{\mathrm{e}}\right)$ is used as a normalization factor for both the radius and velocity dispersion. An important aspect to consider during the fitting process was the effect of the PSF in our measurements. We account for this effect by convolving our models for each galaxy with the CALIFA PSF during the fitting process. As illustrated in the comparison with the ATLAS ${ }^{3 \mathrm{D}}$ survey data (see Sect. 4.3), our velocity dispersions are probably smaller than they should at the very center of galaxies. Ignoring this effect artificially lowers the $\alpha$ parameter in the power-law function.

Figure 10 shows the individual profiles for classes 1 and 3 . Class 1 , in the top panel, is made of predominantly early-type systems with an average stellar mass of $\sim 10^{11} M_{\odot}$ and absolute magnitude $M_{\mathrm{r}} \sim-22 \mathrm{mag}$. We determined the average profile for the class by weighting with the volume correction factor $\left(V_{\max }^{-1}\right)$ of each galaxy. That provides a good representation of the average profile for early-type galaxies with those properties. The average fit and uncertainty is indicated with the solid red line and black dashed lines, respectively. The average value of -0.055 is in good agreement with corrections reported in previous works (e.g., $\alpha=-0.04$, Jorgensen et al. 1995; $\alpha=-0.06$, Mehlert et al. 2003; $\alpha=-0.066$, Cappellari et al. 2006), but see Ziegler \& Bender (1997) for a steeper correction using a different prescription.

The family of late-type systems in class 3 is much more heterogeneous. We decided to divide the sample into three intervals of mass and absolute magnitude. As for early-type galaxies, PSF effects and volume corrections were taken into account for the fitting. As illustrated in Fig. 10 (bottom panel) there are significant differences in the slopes as a function of mass and magnitude. Our results indicate that low-mass and/or lowluminosity spiral galaxies display larger $\alpha$ values than high-mass and/or bright systems.

\section{Summary and conclusions}

In this paper we present stellar kinematic maps for a sample of 300 galaxies that are part of the CALIFA survey. The sample covers a wide range of Hubble types, from ellipticals to late-spiral galaxies. This subset is a good representation of the CALIFA mother sample in terms of redshift, isophotal diameter, and absolute magnitude. The large footprint of the PPak IFU, together with the average distance of the survey, allow us to measure stellar kinematics well beyond $1.8 R_{\mathrm{e}}$ for $50 \%$ of the galaxies, reaching out to $4-5 R_{\mathrm{e}}$ in a few exceptional cases. The penalty, caused by the combination of spatial sampling and distance, is the inability to detect kinematically decoupled components at the centers of galaxies. Still our data is well suited for the study of large-scale kinematic twists or long-axis rotation, which occurs in a handful of objects.

The measurements presented in this paper are in good agreement with those of other well-known IFU surveys (e.g., ATLAS $^{3 \mathrm{D}}$ and DiskMass). The detailed comparison with the DiskMass survey allowed us to establish that we can measure reliable velocity dispersion values down to $\sigma \sim 40 \mathrm{~km} \mathrm{~s}^{-1}$ (i.e., $\sim 30 \mathrm{~km} \mathrm{~s}^{-1}$ below the instrumental resolution). We also characterized the relative uncertainties of our measurements, which are around $5 \%$ for $\sigma \geq 150 \mathrm{~km} \mathrm{~s}^{-1}$. Below that value, relative uncertainties increase up to $50 \%$ for velocity dispersions all the way down to $\sigma \sim 20 \mathrm{~km} \mathrm{~s}^{-1}$.

We also took advantage of our large sample to compute integrated stellar velocity dispersion aperture corrections for different sets of galaxies across the Hubble sequence. These corrections are particularly useful to homogenize dispersion values of galaxies at different distances. We find two main classes of integrated aperture radial profiles: steadily decreasing profiles representative of early-type galaxies, and a second class of systematically increasing profiles typical of late-type spiral galaxies. We provide aperture corrections for each class for different stellar masses and absolute magnitudes.

The main properties of the sample and the stellar velocity and velocity dispersion maps introduced in this paper are available in Table B.1 and Appendix A. The values of the maps themselves, together with many diagnostic parameters to assess the quality of the measurements, will be made available to the community at the CALIFA website (http://califa.caha.es).

Acknowledgements. We would like to thank the anonymous referee for constructive comments that helped improve some aspects of the original manuscript. We are also grateful to the DiskMass survey team for sharing their data with us for the spectral resolution tests, and to Marc Verheijen and Kyle Westfall in particular for in-depth discussions on the topic. This study makes use of the data provided by the Calar Alto Legacy Integral Field Area (CALIFA) survey (http://califa.caha.es). Based on observations collected at the Centro Astronómico Hispano Alemán (CAHA) at Calar Alto, operated jointly by the Max-Planck-Institut für Astronomie and the Instituto de Astrofísica de Andalucía (CSIC). CALIFA is the first legacy survey being performed at Calar Alto. The CALIFA collaboration would like to thank the IAA-CSIC and MPIA-MPG as major partners of the observatory, and CAHA itself, for the unique access to telescope time and support in manpower and infrastructures. The CALIFA collaboration thanks also the CAHA staff for the dedication to this project.

Funding and financial support acknowledgements: J. F.-B. from grant AYA201348226-C3-1-P from the Spanish Ministry of Economy and Competitiveness (MINECO). J.F.-B. and G.v.d.V. from the FP7 Marie Curie Actions of the European Commission, via the Initial Training Network DAGAL under REA grant agreement number 289313. J.M.-A. and V.W. acknowledge support from the European Research Council Starting Grant (SEDMorph P.I. V. Wild). P.S.-B. acknowledge financial support from the BASAL CATA Center for Astrophysics and Associated Technologies through grant PFB-06. R.M.G.D. from grant AYA2014-57490-P. R.G.-B, R.M.G.D. and E.P. acknowledge support from the project JA-FQM-2828. C.J.W. acknowledges support through the Marie Curie Career Integration Grant 303912. L.G. from the Ministry of Economy, Development, and Tourism's Millennium Science Initiative through grant IC120009 awarded to The Millennium Institute of Astrophysics (MAS), and CONICYT through FONDECYT grant 3140566. I.M. from grant AYA2013-42227-P.

\section{References}

Abazajian, K. N., Adelman-McCarthy, J. K., Agüeros, M. A., et al. 2009, ApJS, 182,543

Aguerri, J. A. L., Debattista, V. P., \& Corsini, E. M. 2003, MNRAS, 338, 465 Aguerri, J. A. L., Méndez-Abreu, J., Falcón-Barroso, J., et al. 2015, A\&A, 576, A102

Bacon, R., Adam, G., Baranne, A., et al. 1995, A\&AS, 113, 347

Bacon, R., Copin, Y., Monnet, G., et al. 2001, MNRAS, 326, 23

Bacon, R., Accardo, M., Adjali, L., et al. 2010, in Ground-based and Airborne Instrumentation for Astronomy III, Proc. SPIE, 7735, 773508

Barrera-Ballesteros, J. K., Falcón-Barroso, J., García-Lorenzo, B., et al. 2014, A\&A, 568, A70

Barrera-Ballesteros, J. K., García-Lorenzo, B., Falcón-Barroso, J., et al. 2015, A\&A, 582, A21

Bekeraitè, S., Walcher, C. J., Falcón-Barroso, J., et al. 2016a, A\&A, 593, A114 Bekeraite, S., Walcher, C. J., Wisotzki, L., et al. 2016b, ApJ, 827, L36 Bender, R., Saglia, R. P., \& Gerhard, O. E. 1994, MNRAS, 269, 785 Bershady, M. A., Verheijen, M. A. W., Swaters, R. A., et al. 2010, ApJ, 716, 198 Bertola, F., \& Capaccioli, M. 1975, ApJ, 200, 439 
Bertola, F., Bettoni, D., Rusconi, L., \& Sedmak, G. 1984, AJ, 89, 356 Binney, J. 1978, MNRAS, 183, 501

Blanton, M. R., Hogg, D. W., Bahcall, N. A., et al. 2003, ApJ, 592, 819 Bryant, J. J., Owers, M. S., Robotham, A. S. G., et al. 2015, MNRAS, 447, 2857

Bundy, K., Bershady, M. A., Law, D. R., et al. 2015, ApJ, 798, 7

Cappellari, M., \& Copin, Y. 2003, MNRAS, 342, 345

Cappellari, M., \& Emsellem, E. 2004, PASP, 116, 138

Cappellari, M., Bacon, R., Bureau, M., et al. 2006, MNRAS, 366, 1126

Cappellari, M., Emsellem, E., Krajnović, D., et al. 2011, MNRAS, 413, 813

Chabrier, G. 2003, PASP, 115, 763

Croom, S. M., Lawrence, J. S., Bland-Hawthorn, J., et al. 2012, MNRAS, 421, 872

Davies, R. L., Efstathiou, G., Fall, S. M., Illingworth, G., \& Schechter, P. L. 1983, ApJ, 266, 41

de Zeeuw, P. T., Bureau, M., Emsellem, E., et al. 2002, MNRAS, 329, 513

Emsellem, E., Cappellari, M., Peletier, R. F., et al. 2004, MNRAS, 352, 721

Emsellem, E., Cappellari, M., Krajnović, D., et al. 2007, MNRAS, 379, 401

Emsellem, E., Cappellari, M., Krajnović, D., et al. 2011, MNRAS, 414, 888

Emsellem, E., Krajnović, D., \& Sarzi, M. 2014, MNRAS, 445, L79

Falcón-Barroso, J., Balcells, M., Peletier, R. F., \& Vazdekis, A. 2003, A\&A, 405, 455

Falcón-Barroso, J., Bacon, R., Bureau, M., et al. 2006, MNRAS, 369, 529

Falcón-Barroso, J., Lyubenova, M., \& van de Ven, G. 2015, in Galaxy Masses as Constraints of Formation Models, eds. M. Cappellari, \& S. Courteau, IAU Symp., 311, 78

Fisher, D. 1997, AJ, 113, 950

Gadotti, D. A., Seidel, M. K., Sánchez-Blázquez, P., et al. 2015, A\&A, 584, A90

Ganda, K., Falcón-Barroso, J., Peletier, R. F., et al. 2006, MNRAS, 367, 46

García-Benito, R., Zibetti, S., Sánchez, S. F., et al. 2015, A\&A, 576, A135

García-Lorenzo, B., Márquez, I., Barrera-Ballesteros, J. K., et al. 2015, A\&A, 573, A59

González, J. J. 1993, Ph.D. Thesis, University of California, Santa Cruz, USA

Husemann, B., Jahnke, K., Sánchez, S. F., et al. 2013, A\&A, 549, A87

Illingworth, G. 1977, ApJ, 218, L43

Iodice, E., Coccato, L., Combes, F., et al. 2015, A\&A, 583, A48

Jorgensen, I., Franx, M., \& Kjaergaard, P. 1995, MNRAS, 276, 1341

Kelz, A., Verheijen, M. A. W., Roth, M. M., et al. 2006, PASP, 118, 129

Kobulnicky, H. A., \& Gebhardt, K. 2000, AJ, 119, 1608

Koleva, M., Prugniel, P., Bouchard, A., \& Wu, Y. 2009, A\&A, 501, 1269
Krajnović, D., Cappellari, M., de Zeeuw, P. T., \& Copin, Y. 2006, MNRAS, 366, 787

Krajnović, D., Emsellem, E., Cappellari, M., et al. 2011, MNRAS, 414, 2923

Krajnović, D., Weilbacher, P. M., Urrutia, T., et al. 2015, MNRAS, 452, 2

Lindblad, B. 1927, MNRAS, 87, 553

Martinsson, T. P. K., Verheijen, M. A. W., Westfall, K. B., et al. 2013, A\&A, 557, A130

Mayall, N. U. 1951, Publ. Obs. Michigan, 10, 19

McDermid, R. M., Emsellem, E., Shapiro, K. L., et al. 2006, MNRAS, 373, 906

Mehlert, D., Thomas, D., Saglia, R. P., Bender, R., \& Wegner, G. 2003, A\&A, 407, 423

Méndez-Abreu, J., Aguerri, J. A. L., Falcón-Barroso, J., et al. 2016, A\&A, submitted

Münch, G., \& Münch, L. 1960, ApJ, 131, 253

Oh, K., Sarzi, M., Schawinski, K., \& Yi, S. K. 2011, ApJS, 195, 13

Pedraz, S., Gorgas, J., Cardiel, N., Sánchez-Blázquez, P., \& Guzmán, R. 2002, MNRAS, 332, L59

Pizzella, A., Corsini, E. M., Vega Beltrán, J. C., \& Bertola, F. 2004, A\&A, 424, 447

Roth, M. M., Kelz, A., Fechner, T., et al. 2005, PASP, 117, 620

Rubin, V. C., Waterman, A. H., \& Kenney, J. D. P. 1999, AJ, 118, 236

Ryś, A., Falcón-Barroso, J., \& van de Ven, G. 2013, MNRAS, 428, 2980

Sánchez, S. F., Kennicutt, R. C., Gil de Paz, A., et al. 2012, A\&A, 538, A8

Sánchez, S. F., García-Benito, R., Zibetti, S., et al. 2016, A\&A, 594, A36

Seidel, M. K., Falcón-Barroso, J., Martínez-Valpuesta, I., et al. 2015, MNRAS, 451,936

Simien, F., \& Prugniel, P. 1997, A\&AS, 126, 519

Valdes, F., Gupta, R., Rose, J. A., Singh, H. P., \& Bell, D. J. 2004, ApJS, 152, 251

van den Bosch, R. C. E., Gebhardt, K., Gültekin, K., Yıldırım, A., \& Walsh, J. L. 2015, ApJS, 218, 10

Vega Beltrán, J. C., Pizzella, A., Corsini, E. M., et al. 2001, A\&A, 374, 394

Verheijen, M. A. W., Bershady, M. A., Andersen, D. R., et al. 2004, Astron. Nachr., 325, 151

Walcher, C. J., Wisotzki, L., Bekeraité, S., et al. 2014, A\&A, 569, A1

Westfall, K. B., Bershady, M. A., \& Verheijen, M. A. W. 2011, ApJS, 193, 21

Wild, V., Rosales-Ortega, F., Falcón-Barroso, J., et al. 2014, A\&A, 567, A132

Ziegler, B. L., \& Bender, R. 1997, MNRAS, 291, 527 


\section{Appendix A: Stellar kinematic maps}

This appendix presents all the stellar velocity (Fig. A.1) and velocity dispersion (Fig. A.2) maps extracted from the V1200 grating used in this paper. The complete sample comprises 300 galaxies of Hubble morphological types ranging from ellipticals to late-type spirals. Velocity maps are in $\mathrm{km} \mathrm{s}^{-1}$ and use a fixed range in the interval $[-150,150] \mathrm{km} \mathrm{s}^{-1}$. Velocity dispersion maps are also expressed in $\mathrm{km} \mathrm{s}^{-1}$ and use a fixed range from 20 to $300 \mathrm{~km} \mathrm{~s}^{-1}$. Color schemes as in Fig. 3. Overlaid contours come from SDSS $g$-band images and have been limited to the isophote reaching $\sim 2 R_{\mathrm{e}}$. All panels cover an area of $80^{\prime \prime} \times 100^{\prime \prime}$. 
J. Falcón-Barroso et al.: Stellar kinematics across the Hubble sequence

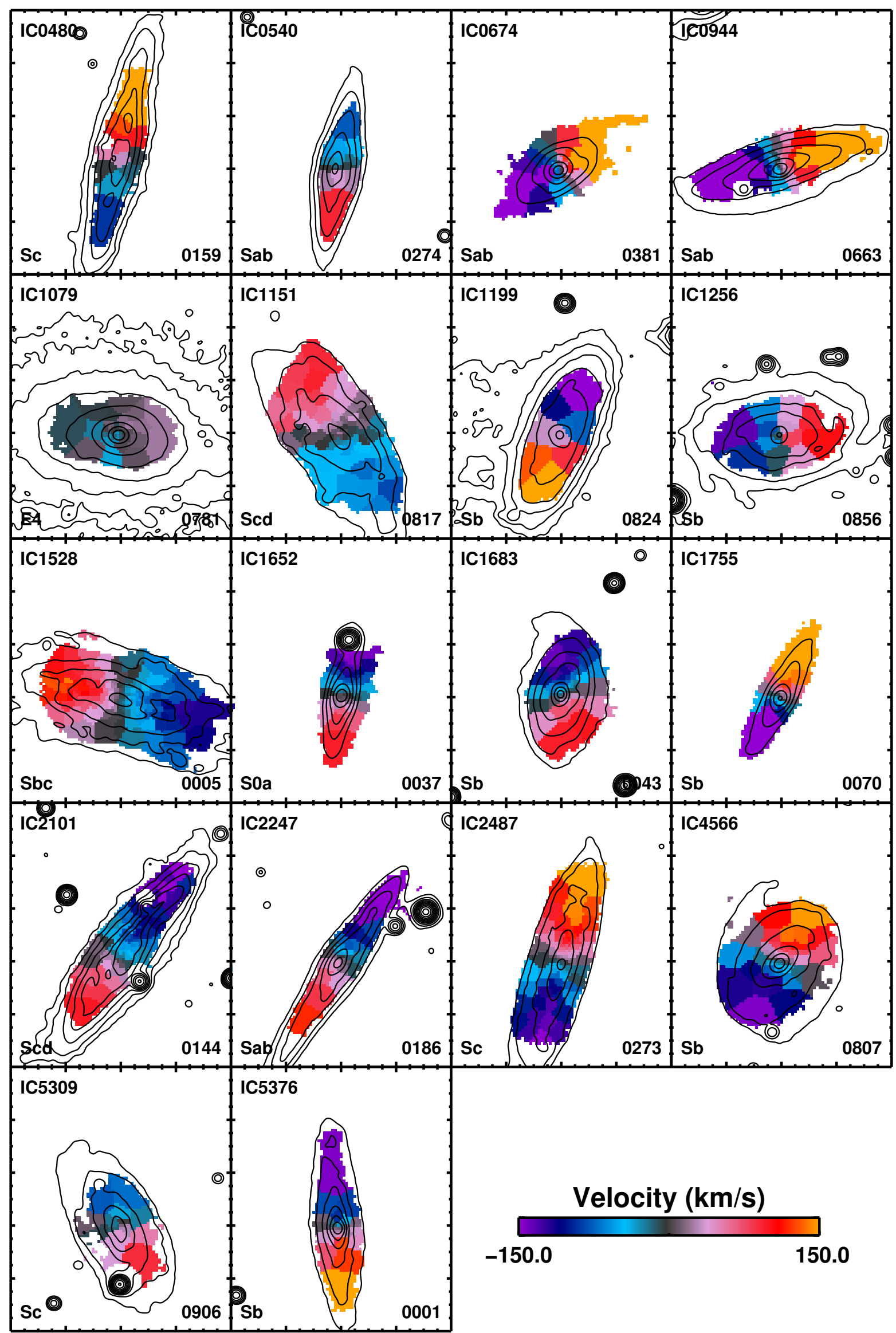

Fig. A.1. Stellar velocity maps from the CALIFA V1200 dataset. 


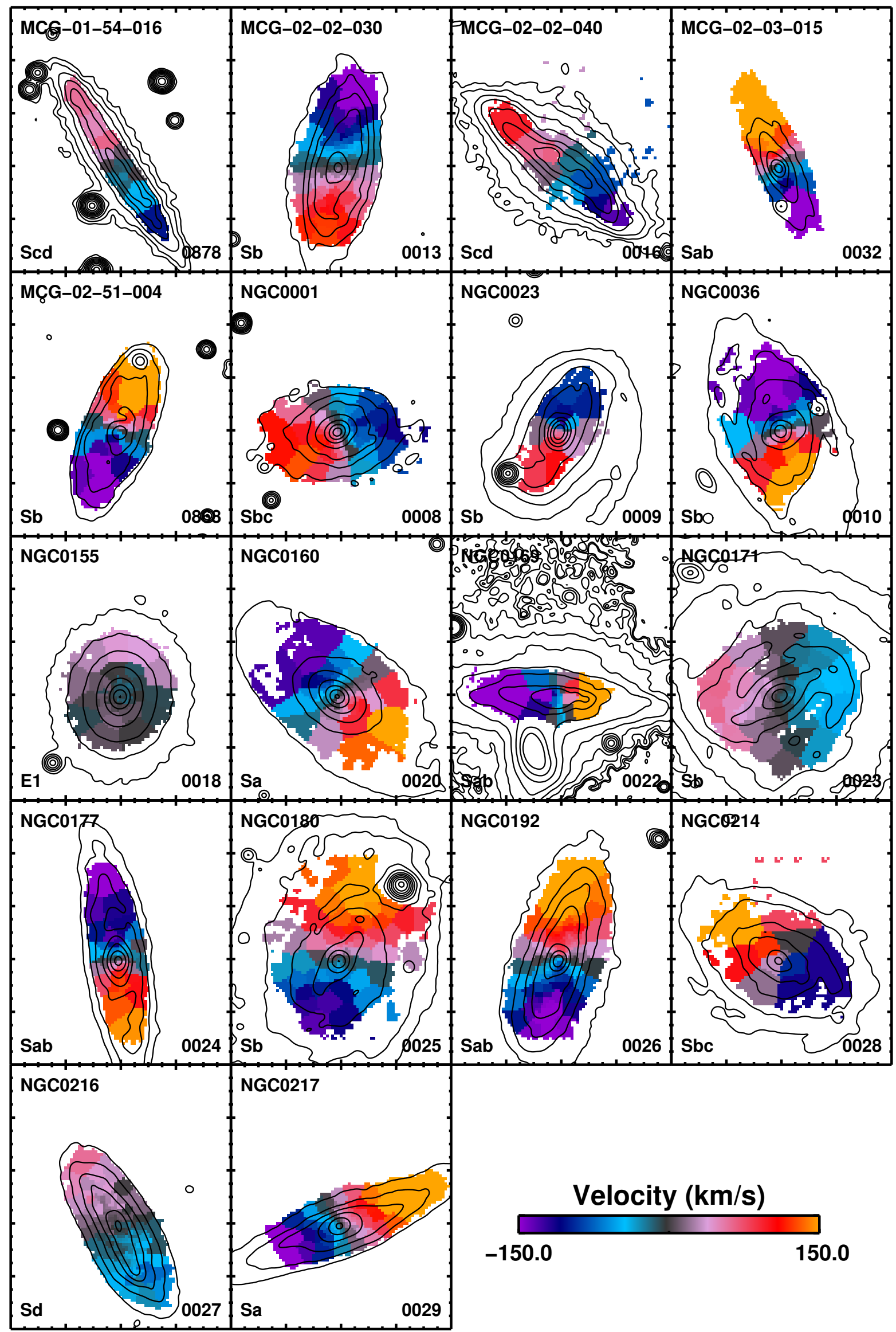

Fig. A.1. continued. 
J. Falcón-Barroso et al.: Stellar kinematics across the Hubble sequence

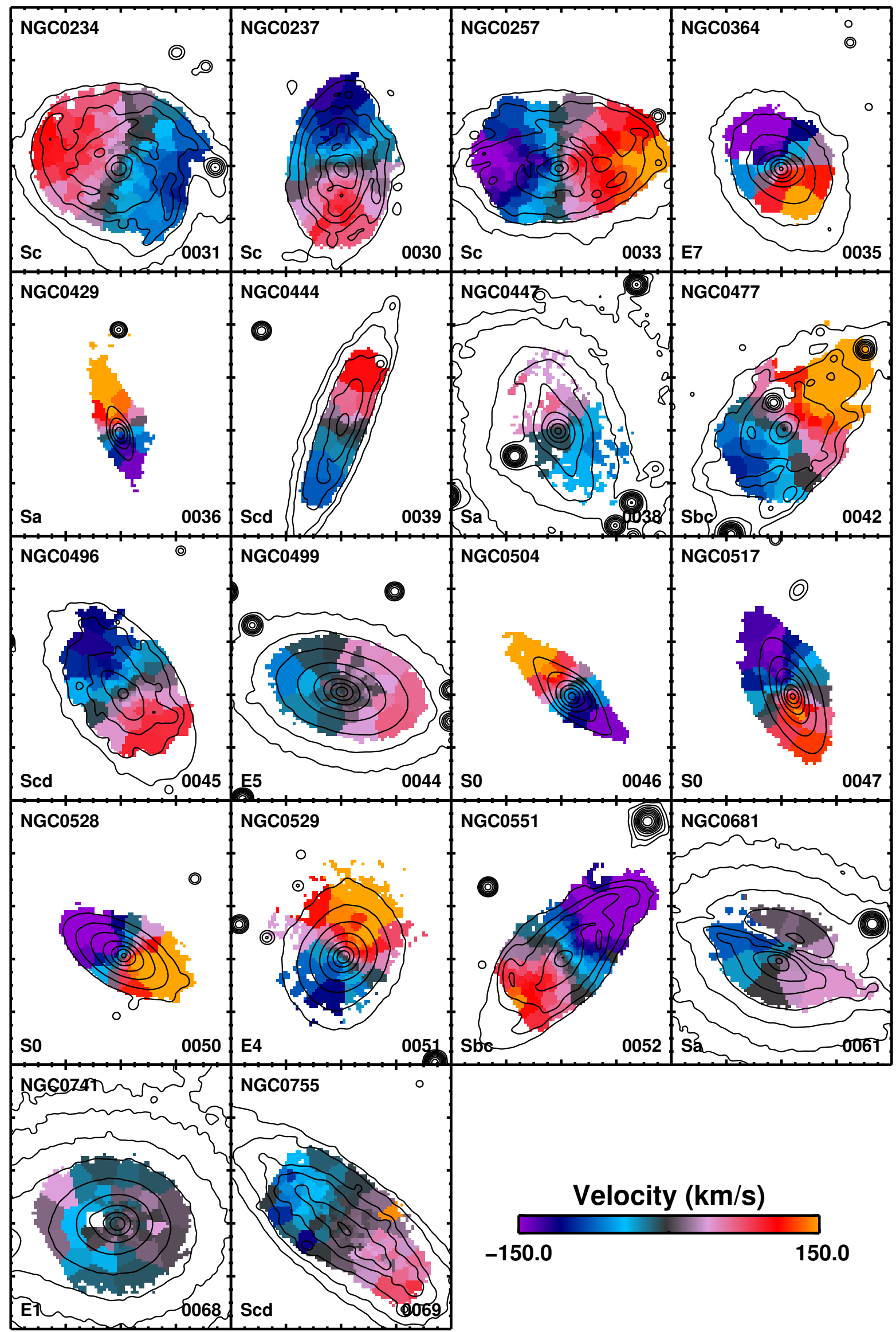

Fig. A.1. continued. 


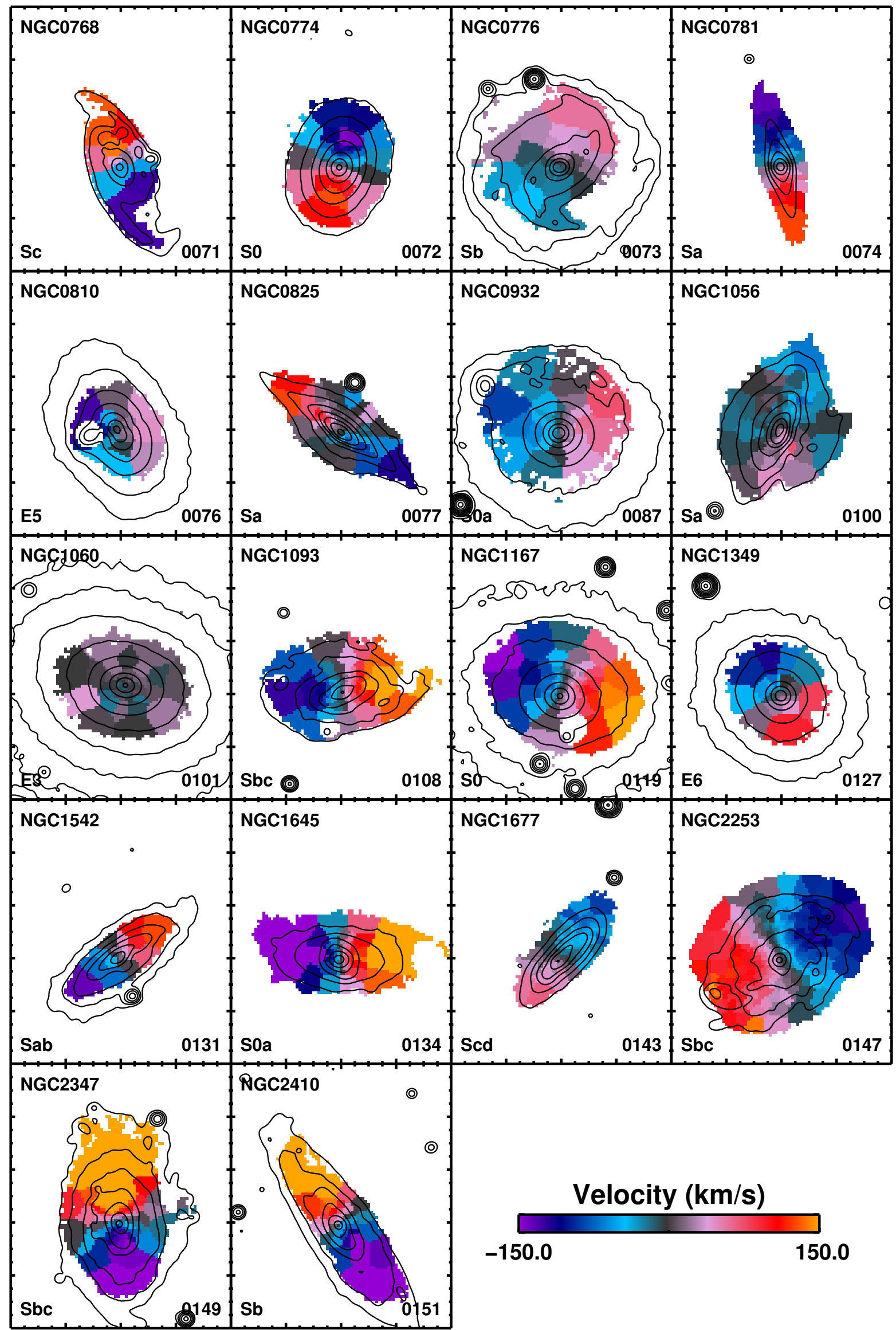

Fig. A.1. continued. 
J. Falcón-Barroso et al.: Stellar kinematics across the Hubble sequence

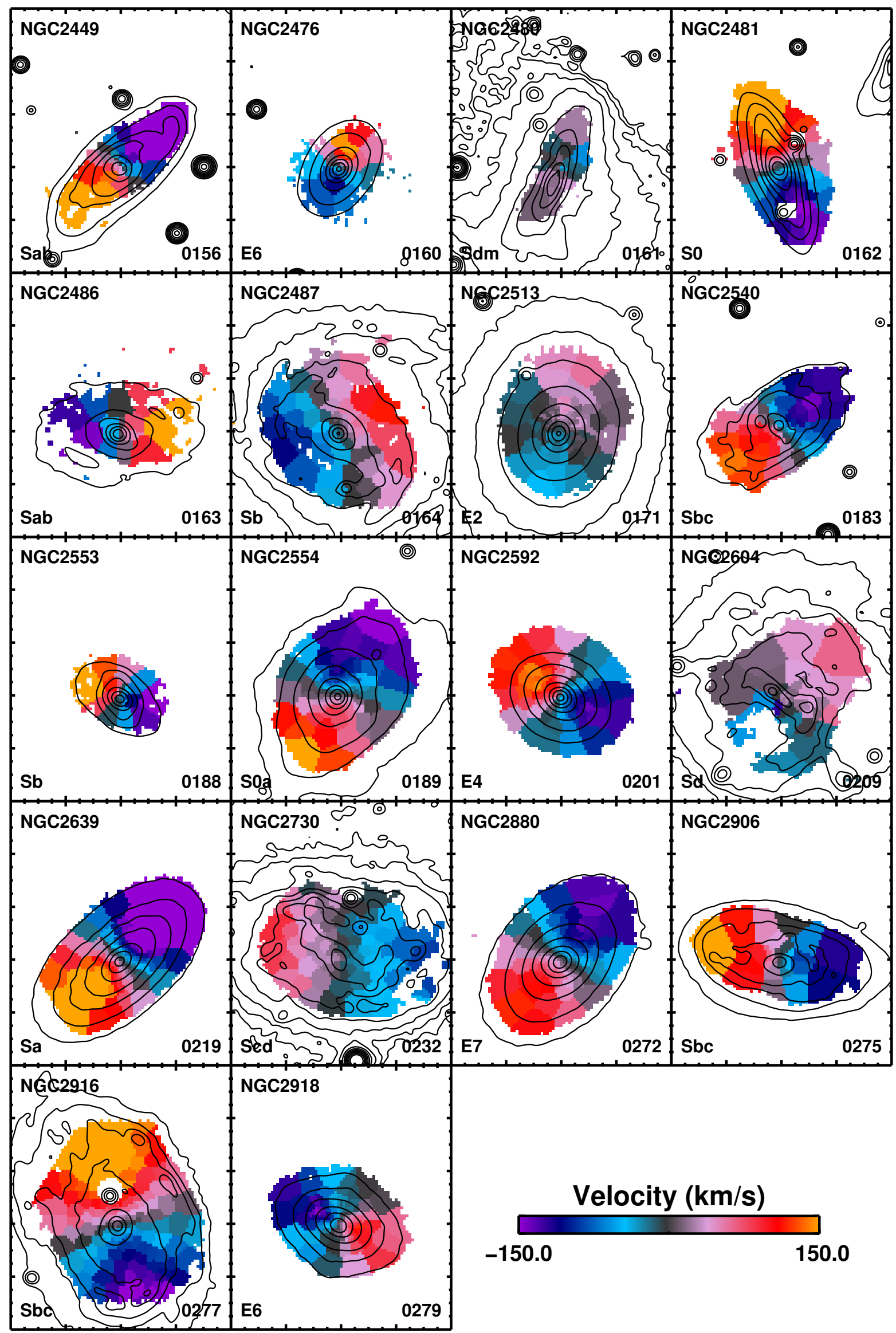

Fig. A.1. continued. 


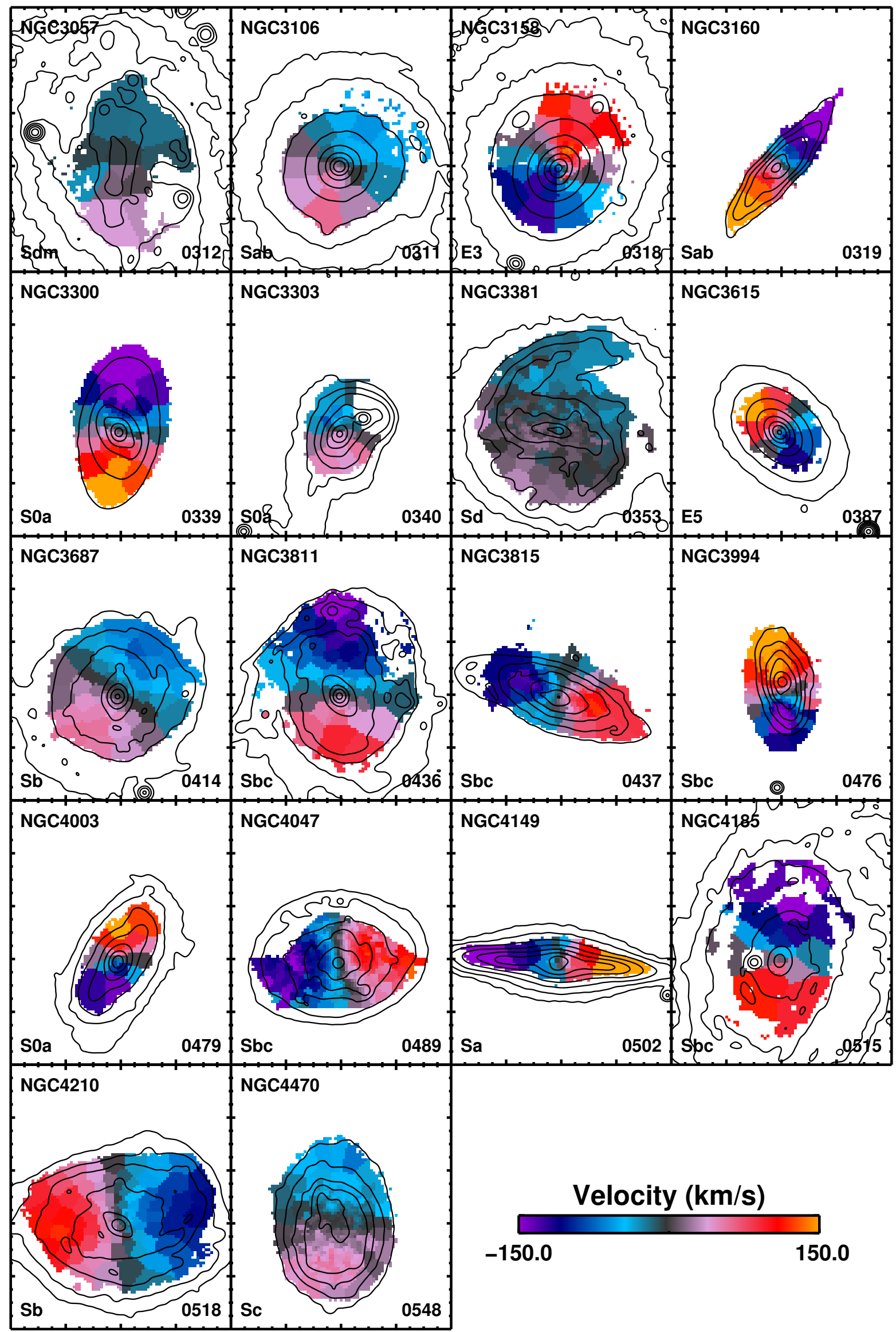

Fig. A.1. continued. 
J. Falcón-Barroso et al.: Stellar kinematics across the Hubble sequence

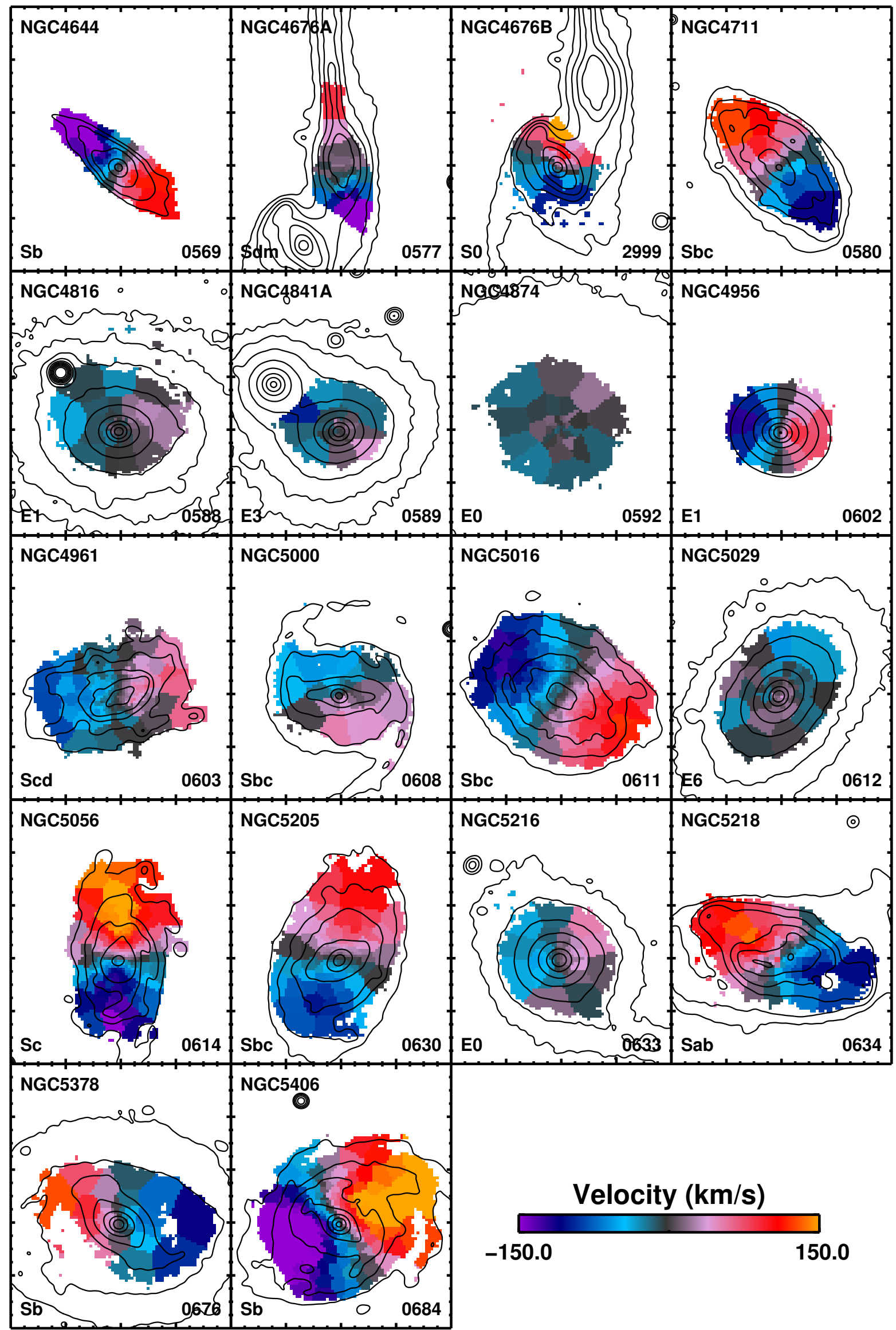

Fig. A.1. continued. 


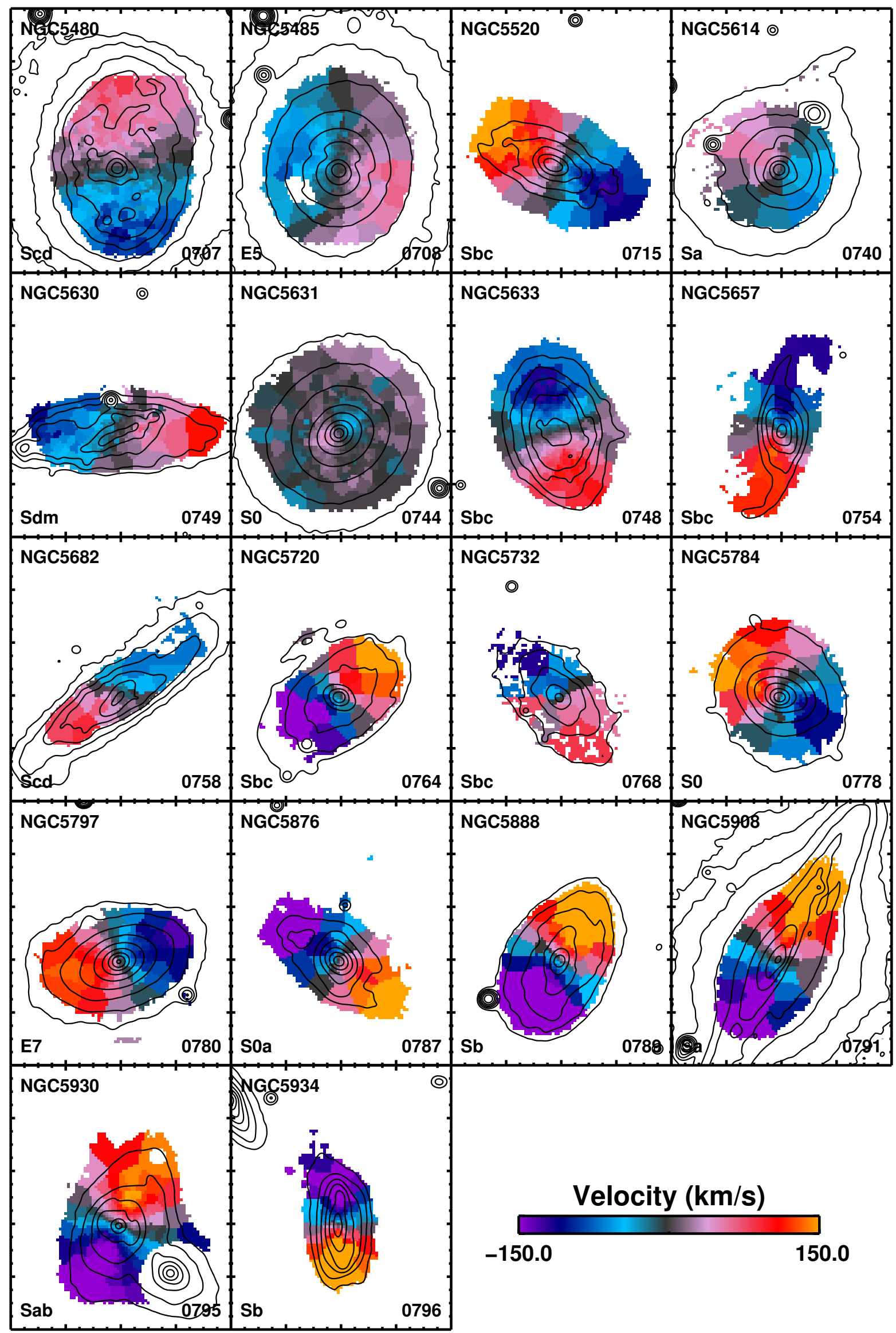

Fig. A.1. continued. 
J. Falcón-Barroso et al.: Stellar kinematics across the Hubble sequence

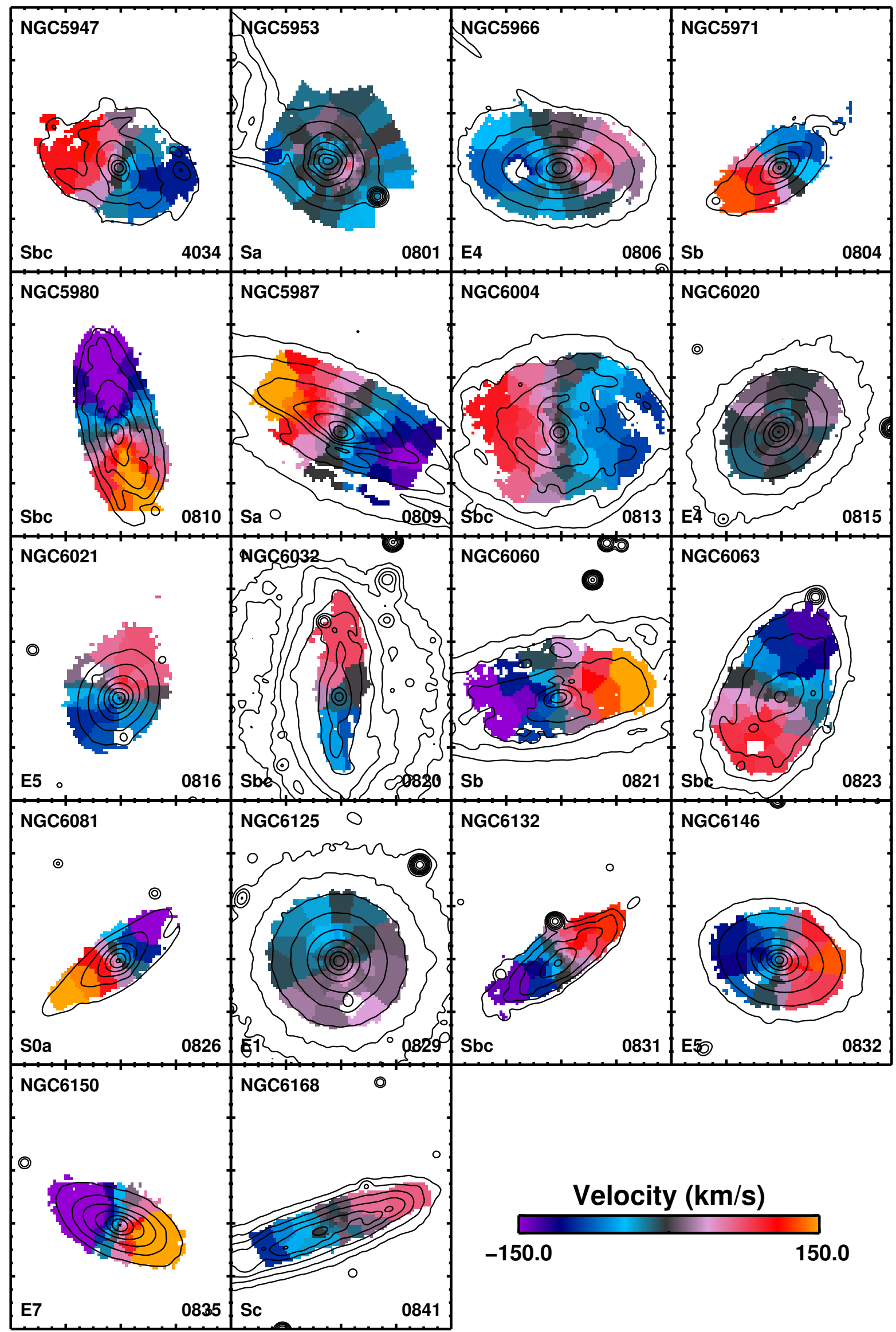

Fig. A.1. continued. 


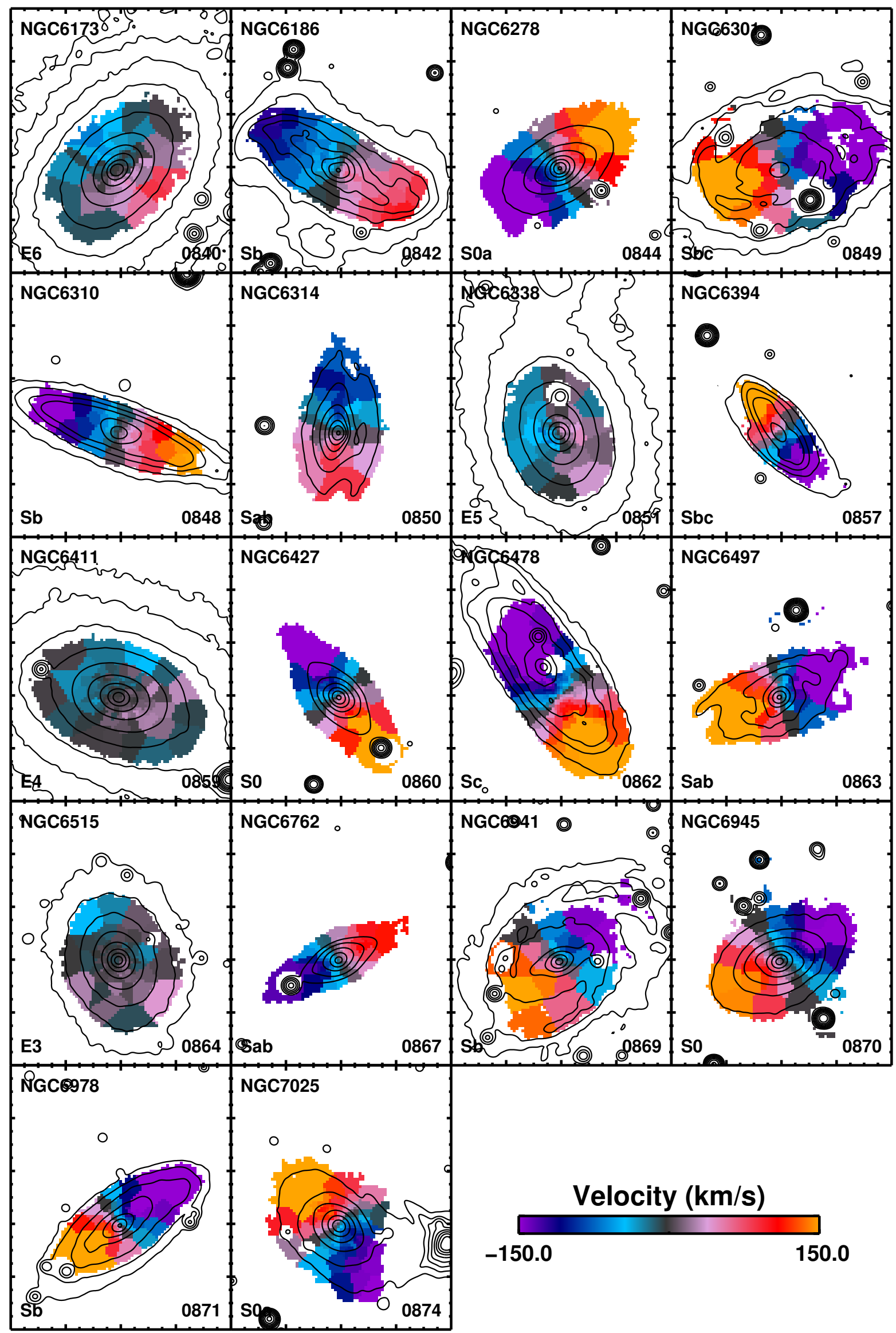

Fig. A.1. continued. 
J. Falcón-Barroso et al.: Stellar kinematics across the Hubble sequence

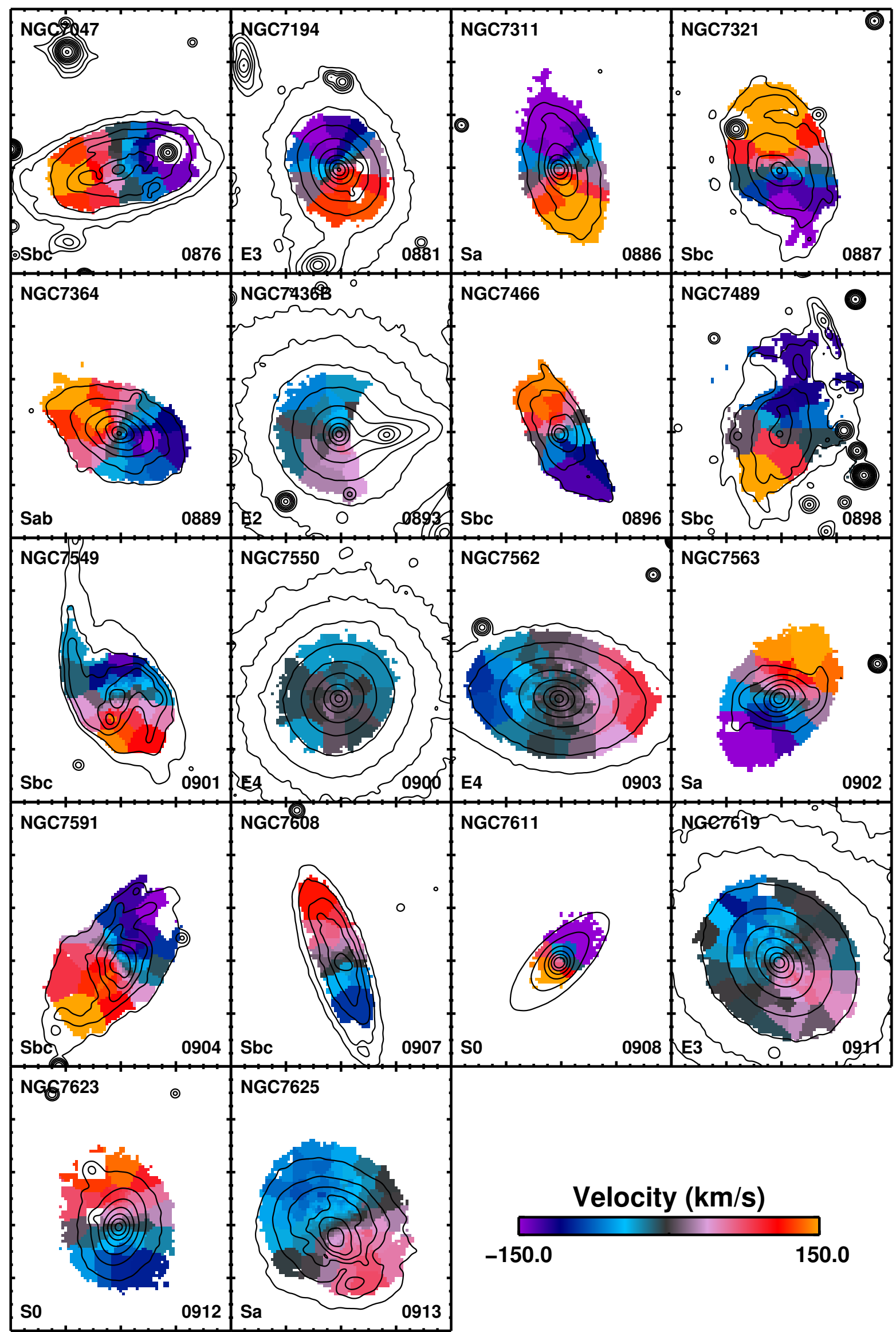

Fig. A.1. continued. 


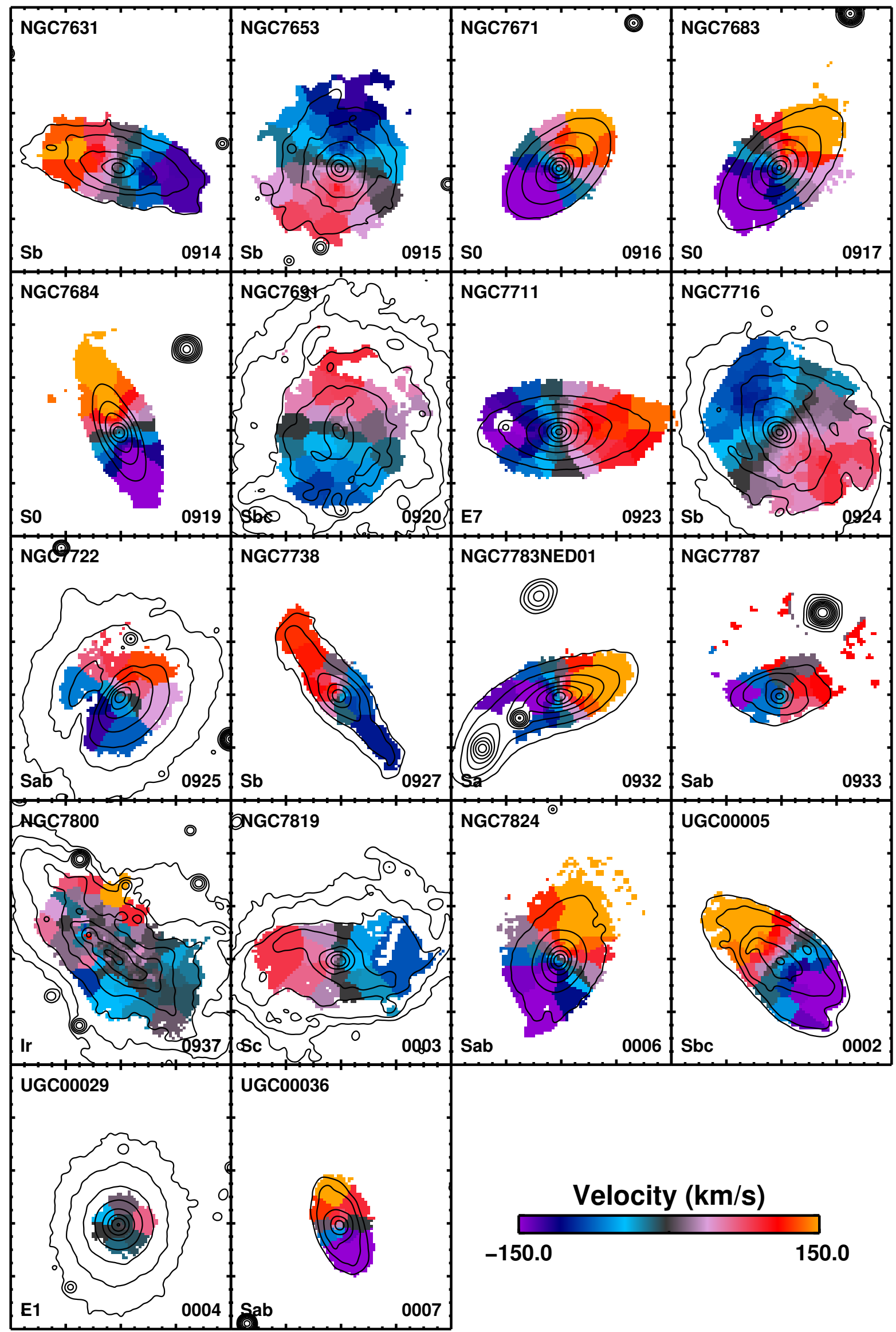

Fig. A.1. continued. 
J. Falcón-Barroso et al.: Stellar kinematics across the Hubble sequence

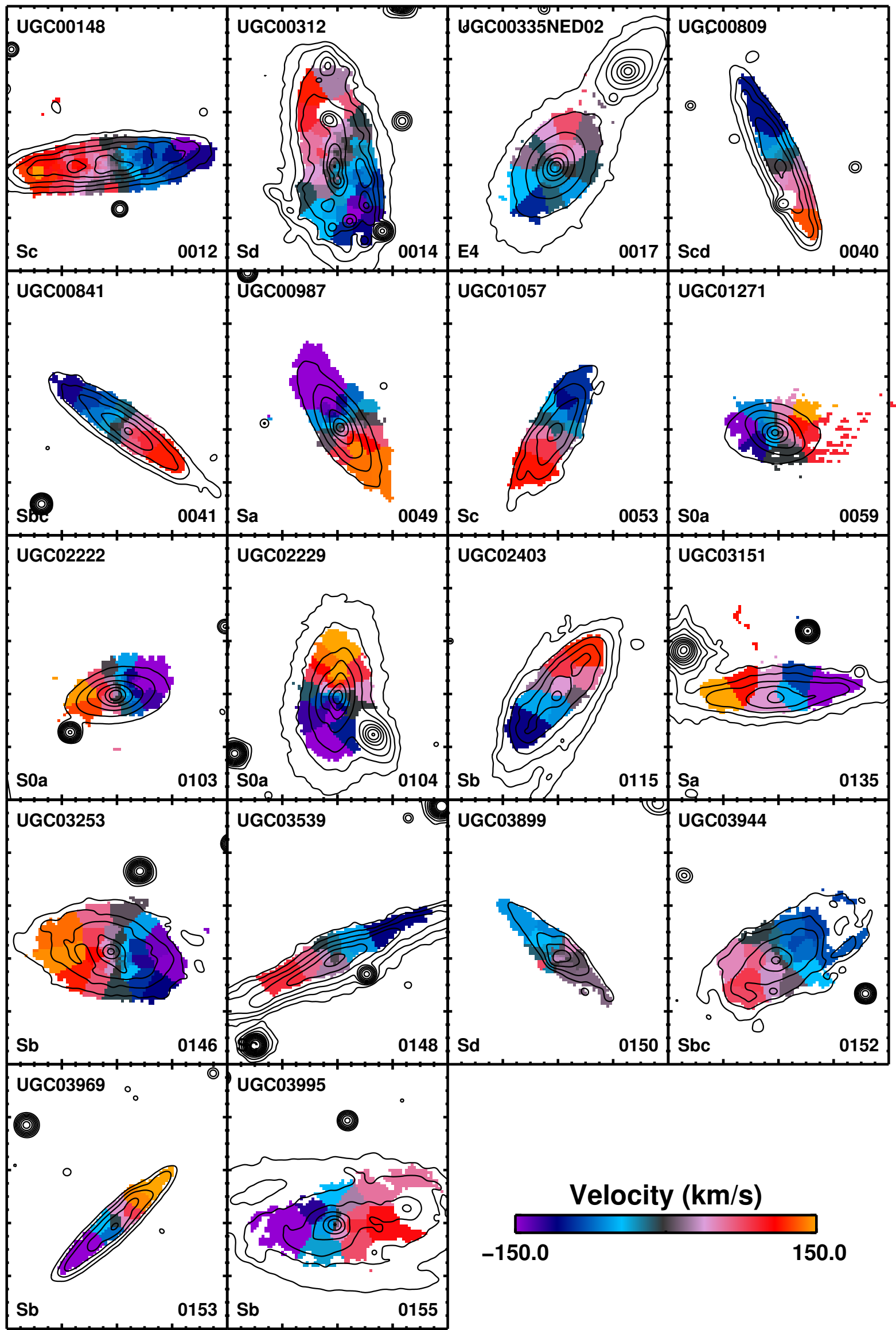

Fig. A.1. continued. 


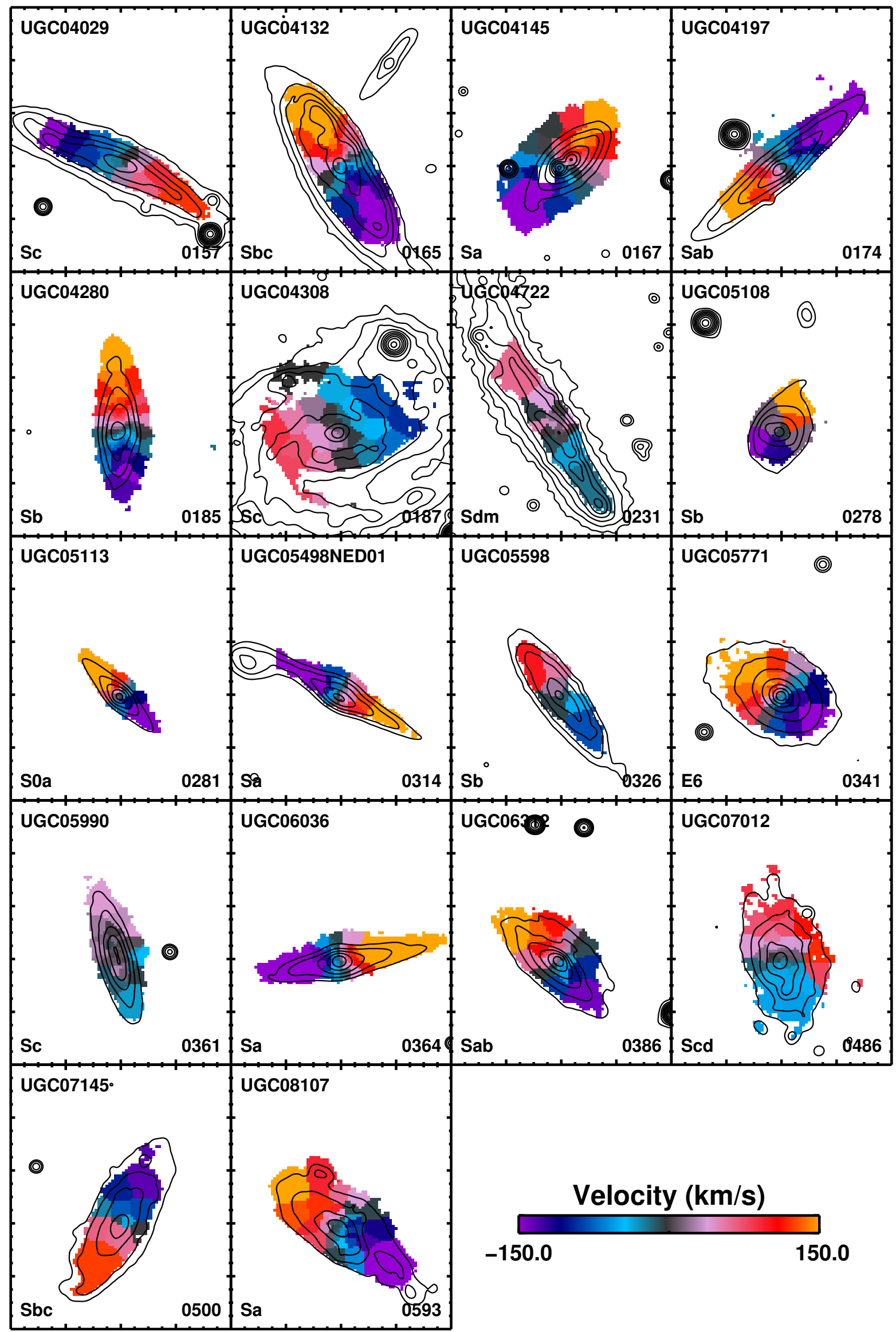

Fig. A.1. continued. 
J. Falcón-Barroso et al.: Stellar kinematics across the Hubble sequence

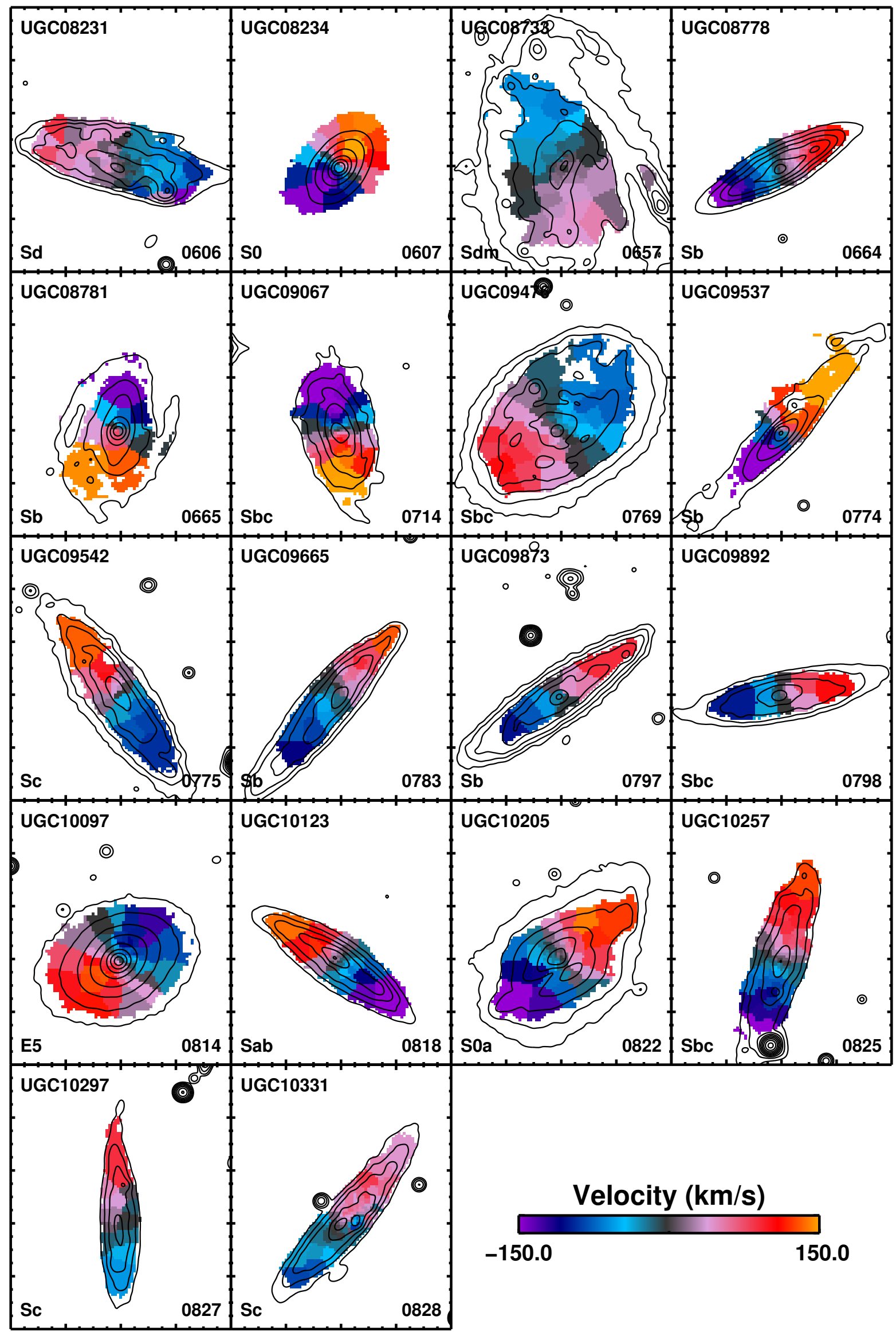

Fig. A.1. continued. 


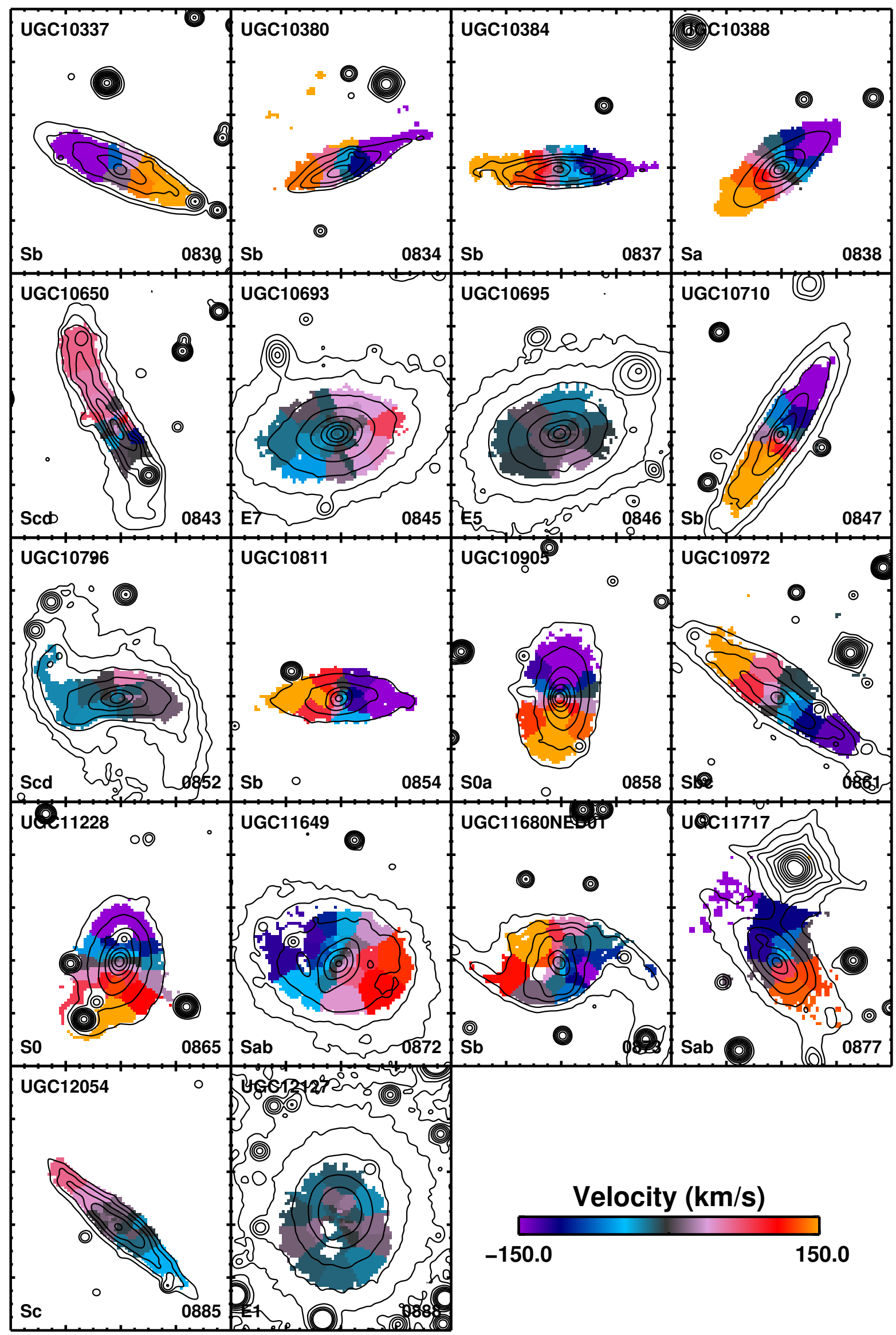

Fig. A.1. continued. 
J. Falcón-Barroso et al.: Stellar kinematics across the Hubble sequence
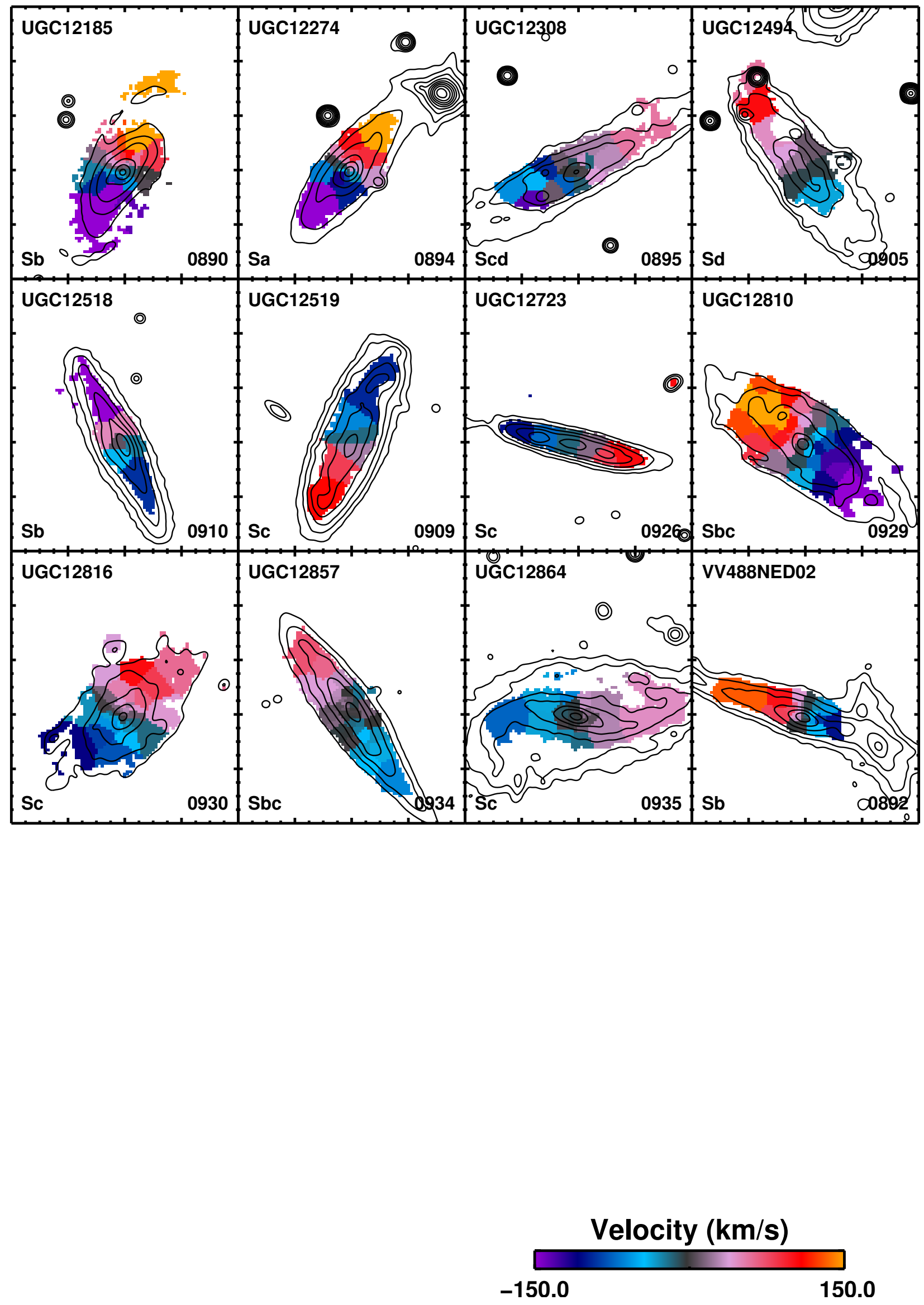

Fig. A.1. continued. 


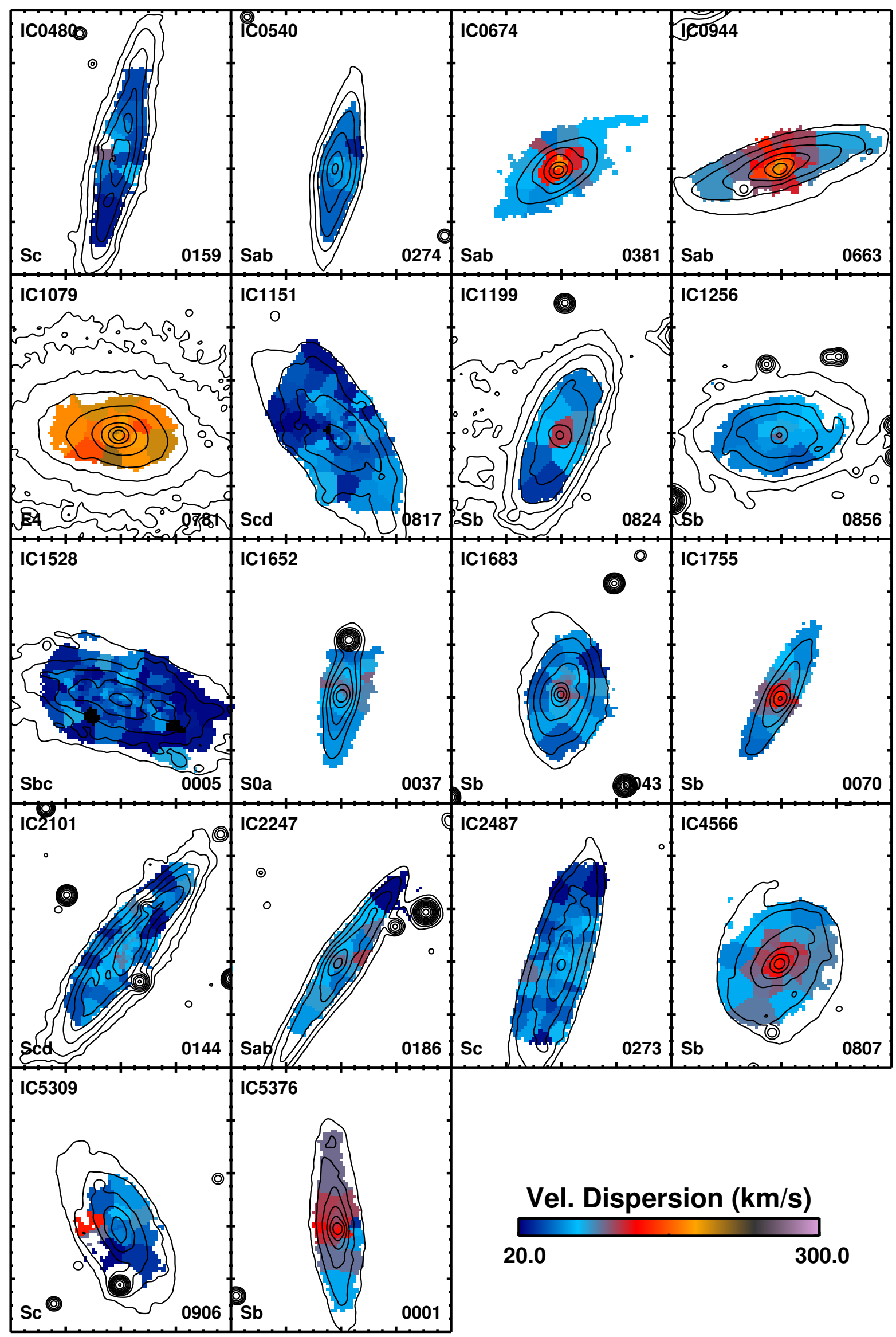

Fig. A.2. Stellar velocity dispersion maps from the CALIFA V1200 dataset. 
J. Falcón-Barroso et al.: Stellar kinematics across the Hubble sequence

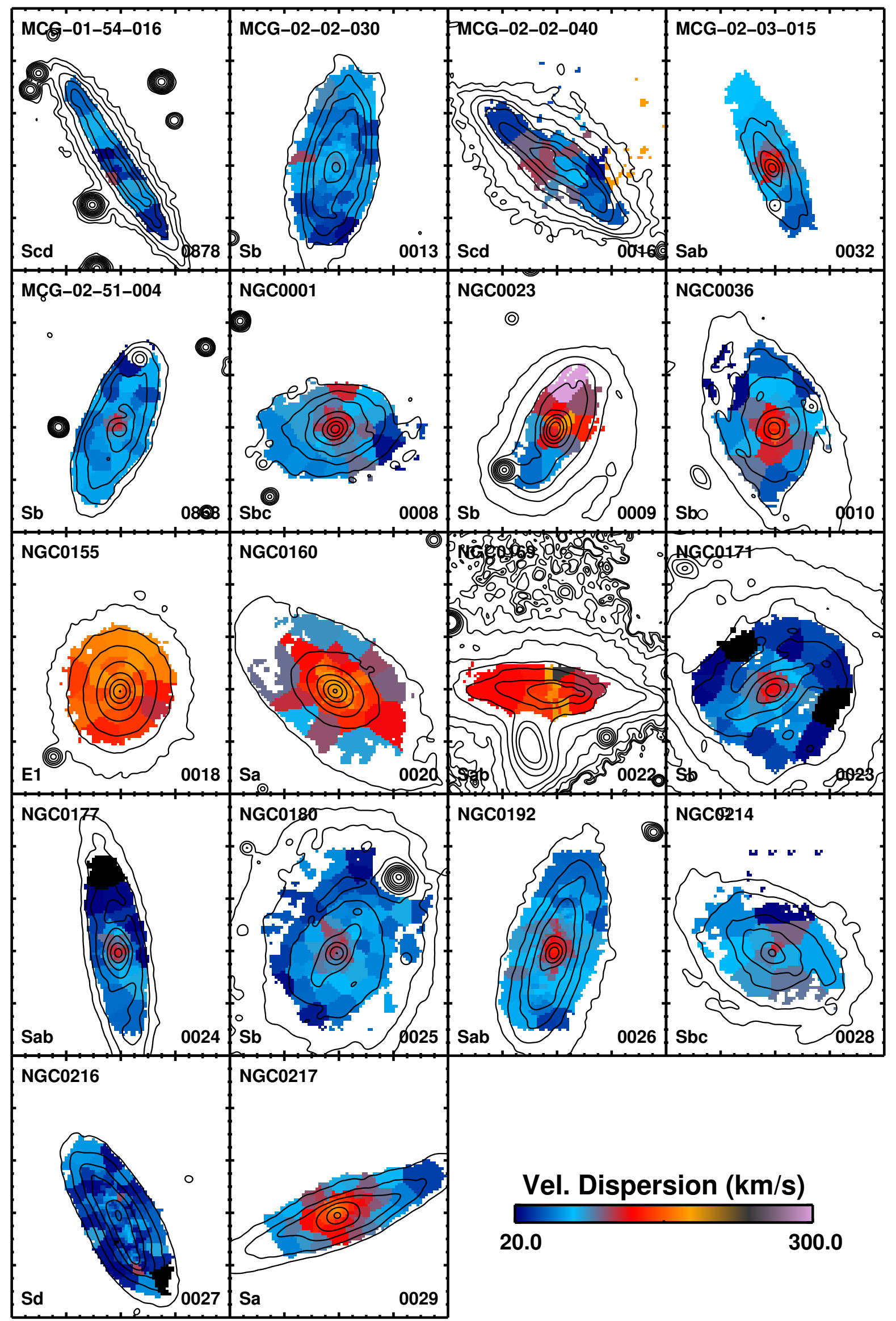

Fig. A.2. continued. 


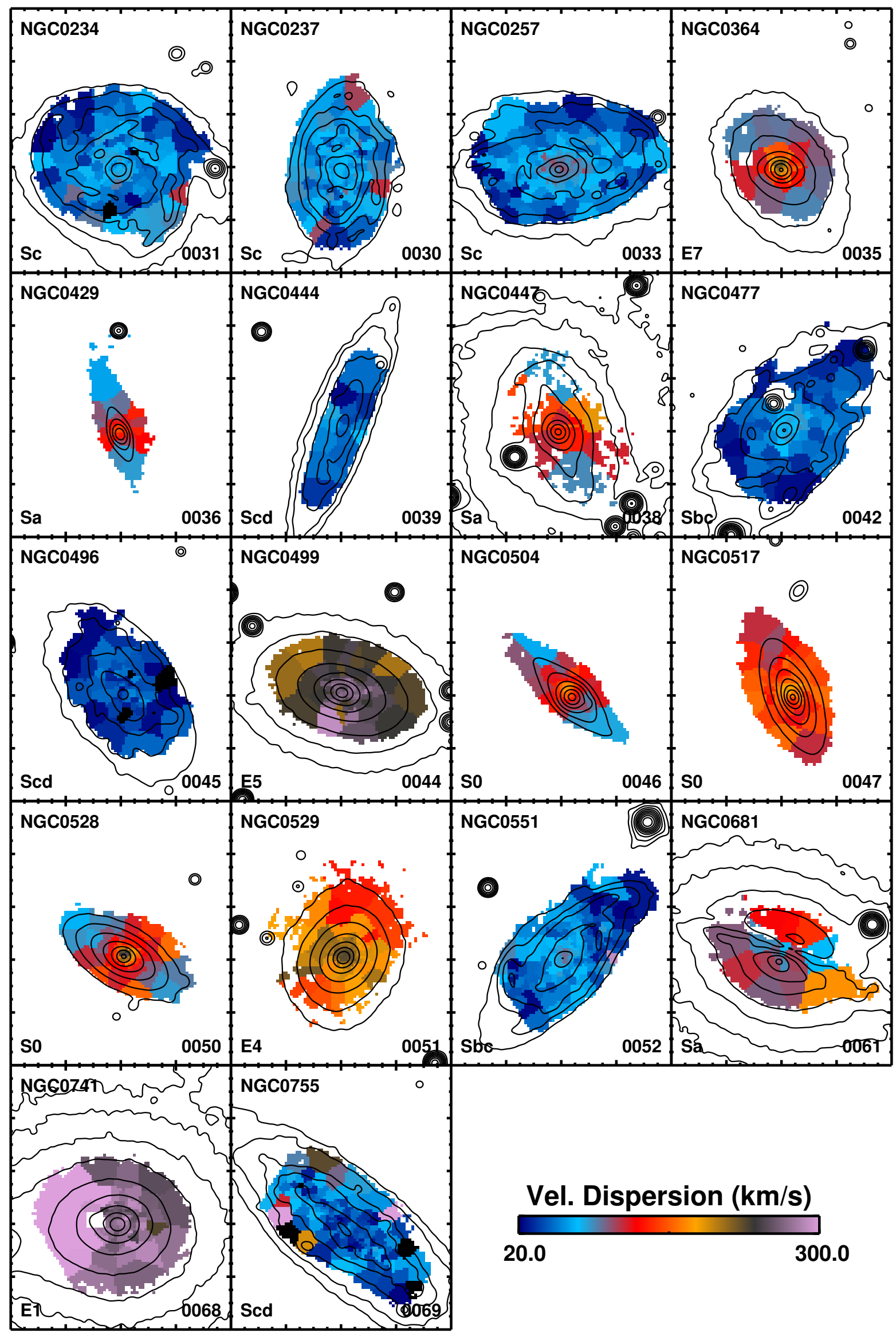

Fig. A.2. continued. 
J. Falcón-Barroso et al.: Stellar kinematics across the Hubble sequence

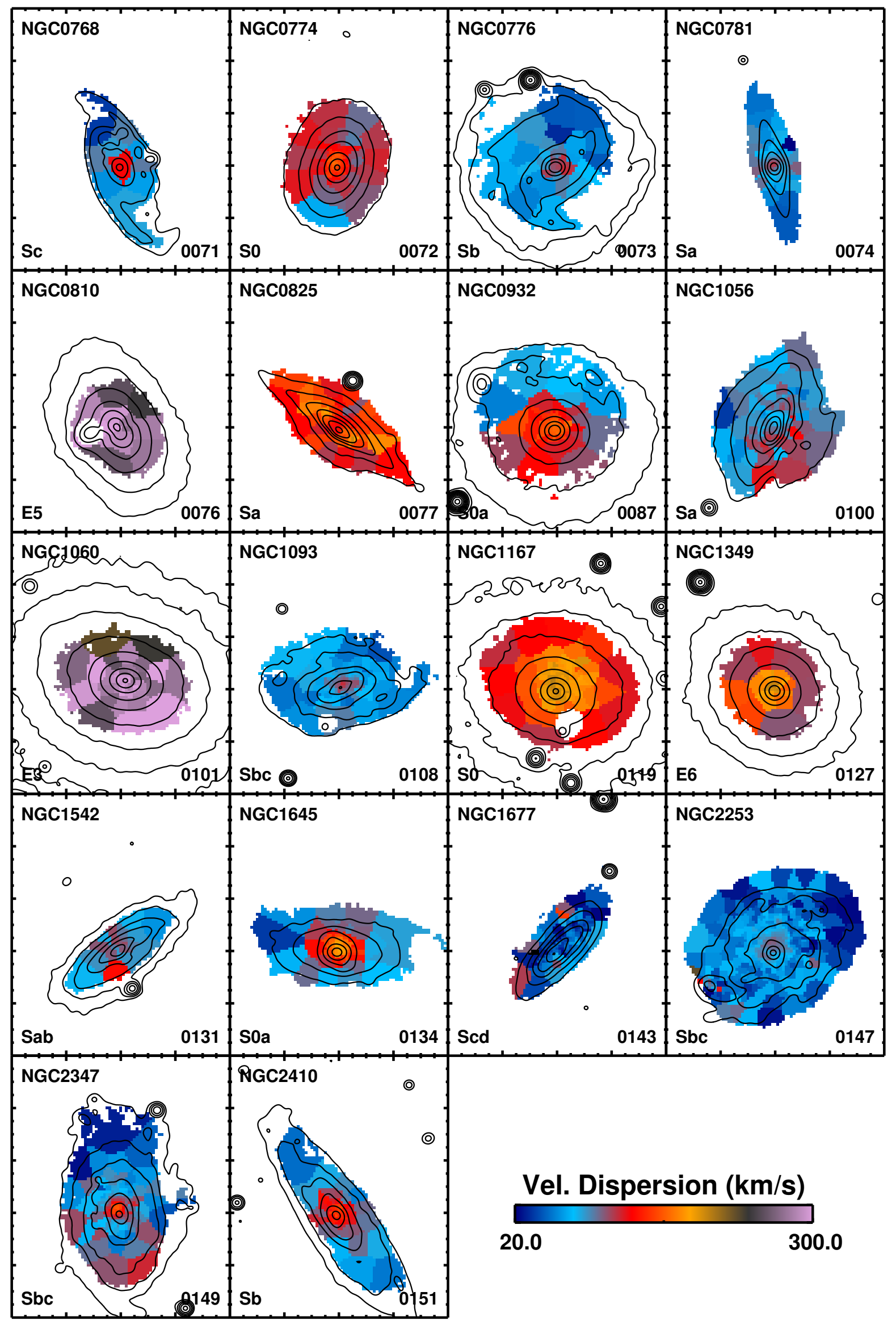

Fig. A.2. continued. 


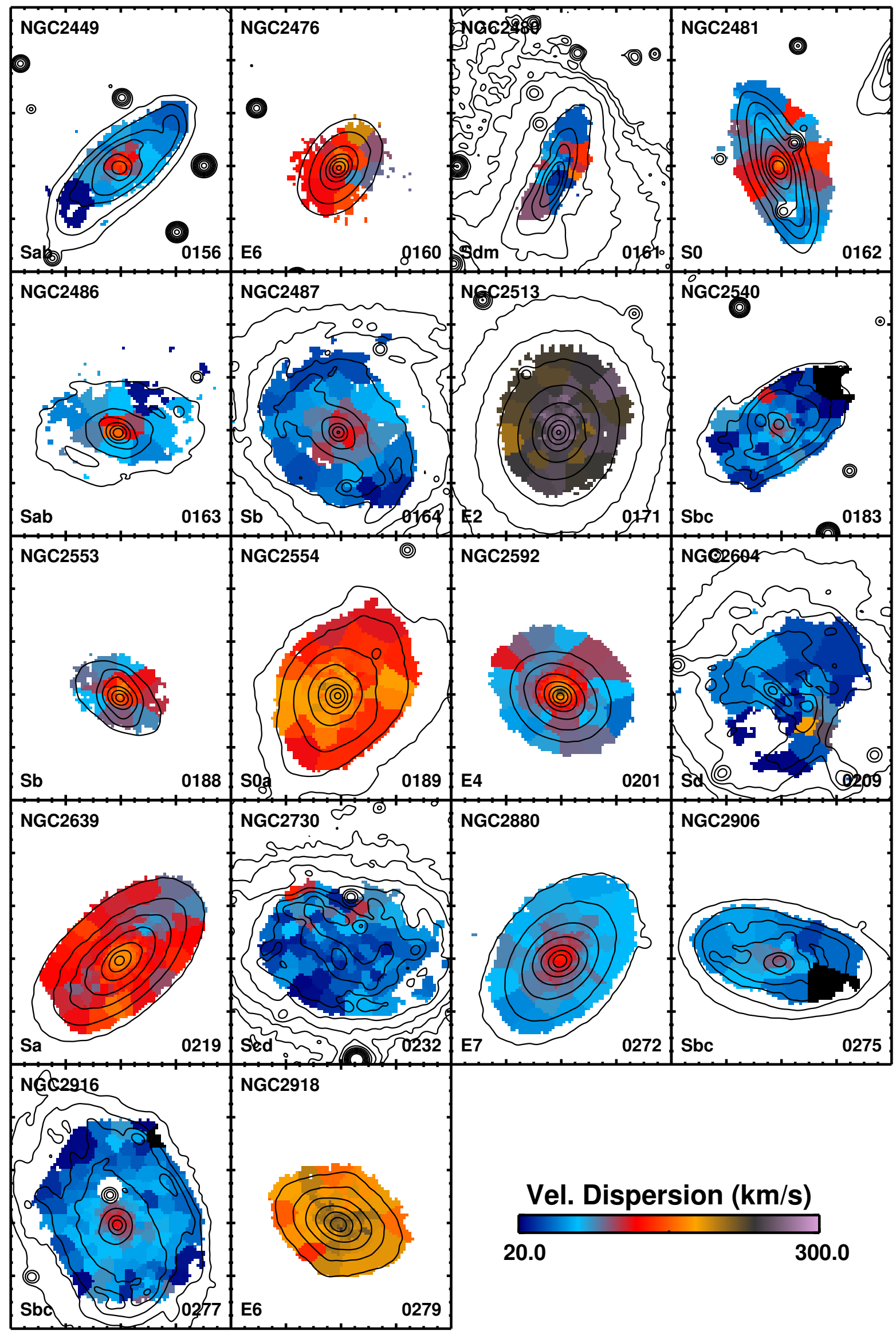

Fig. A.2. continued. 


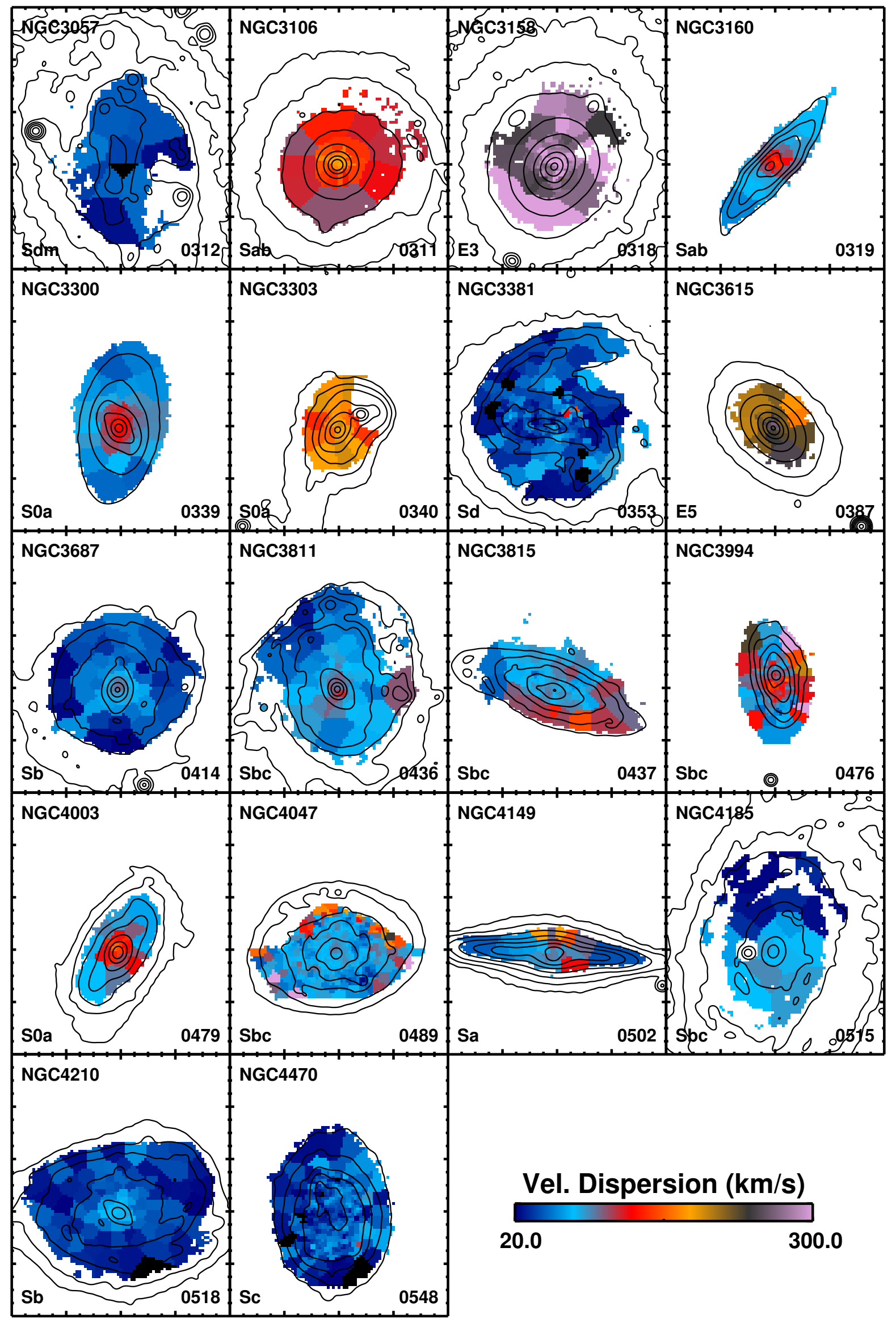

Fig. A.2. continued. 


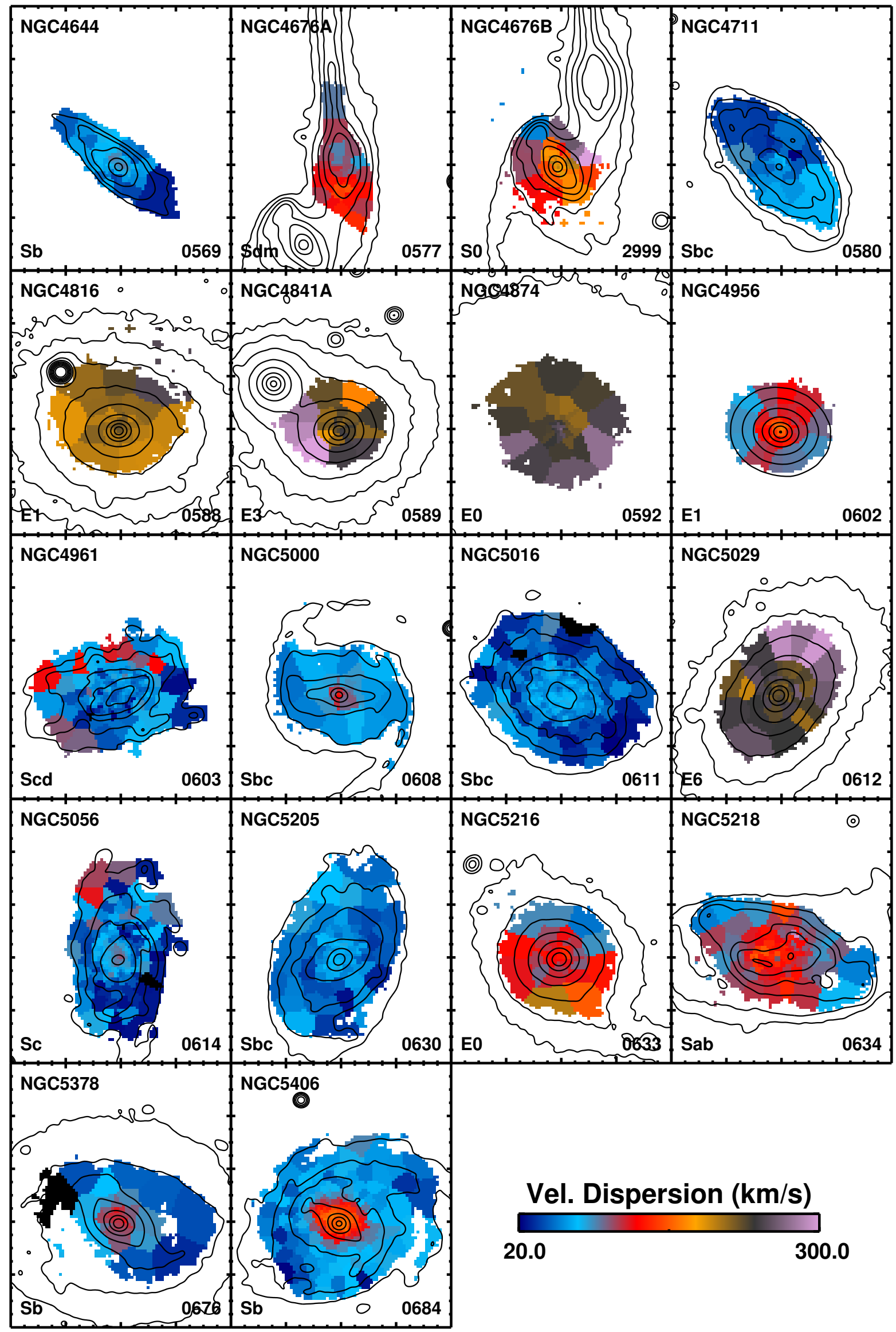

Fig. A.2. continued. 

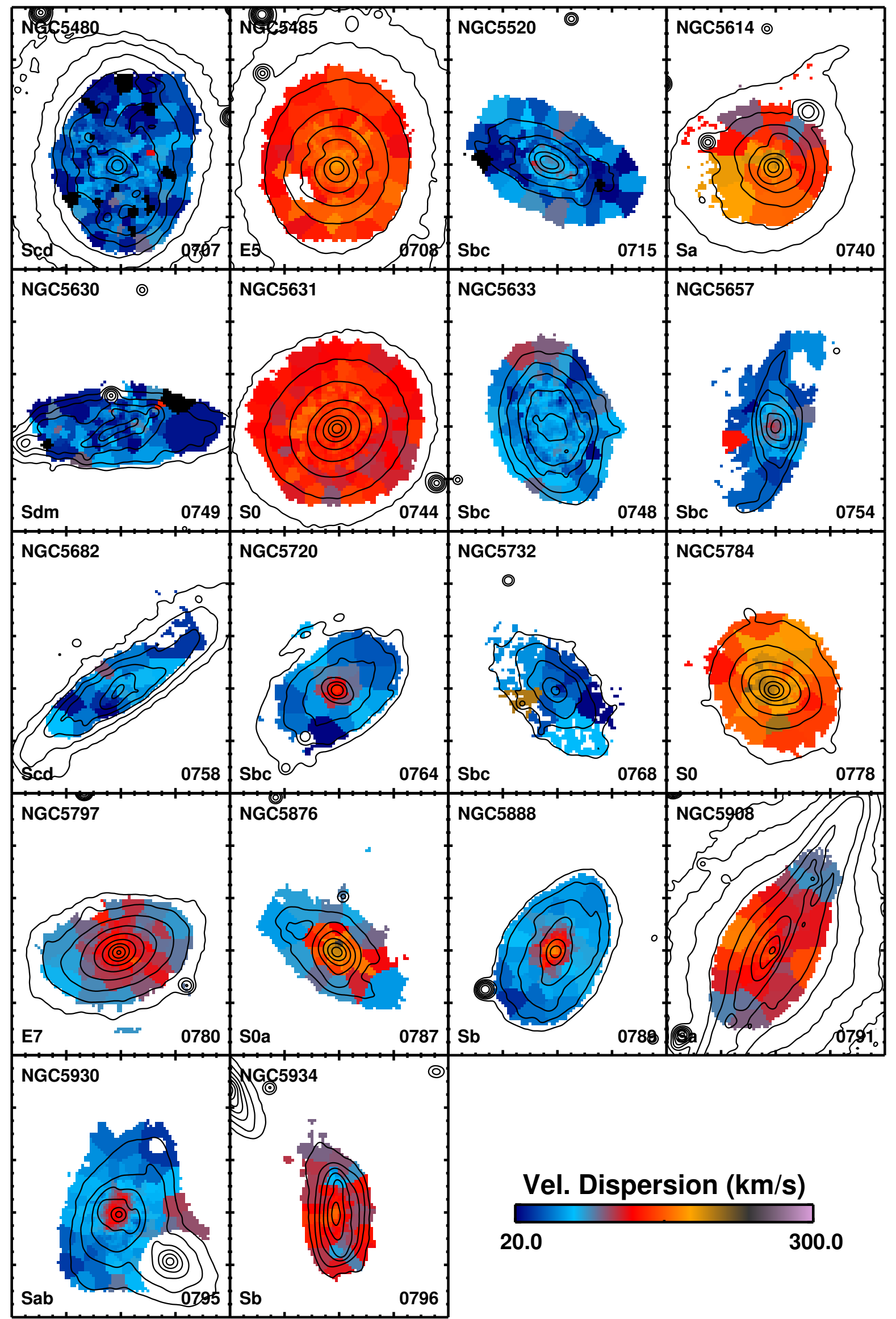

Fig. A.2. continued. 


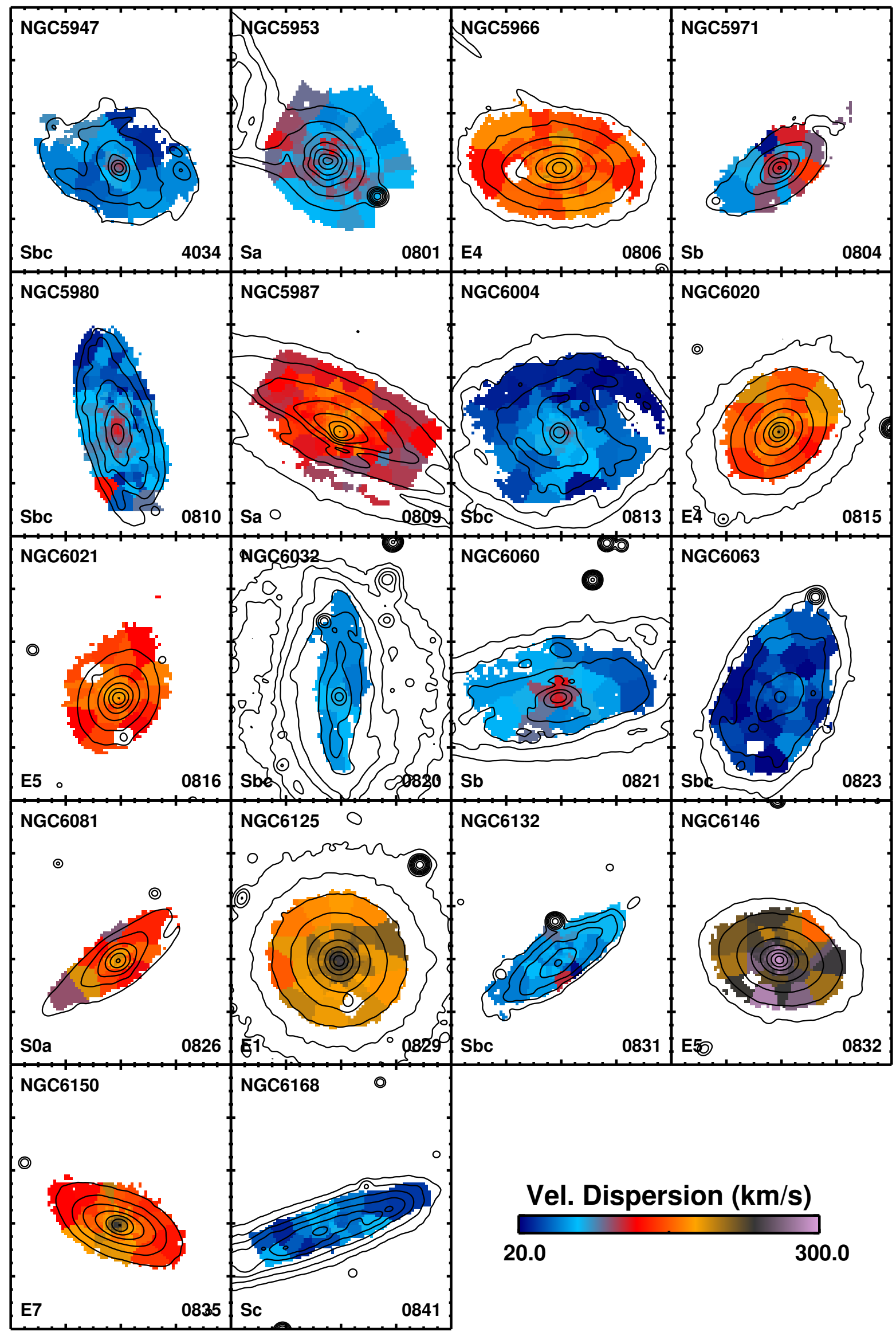

Fig. A.2. continued. 


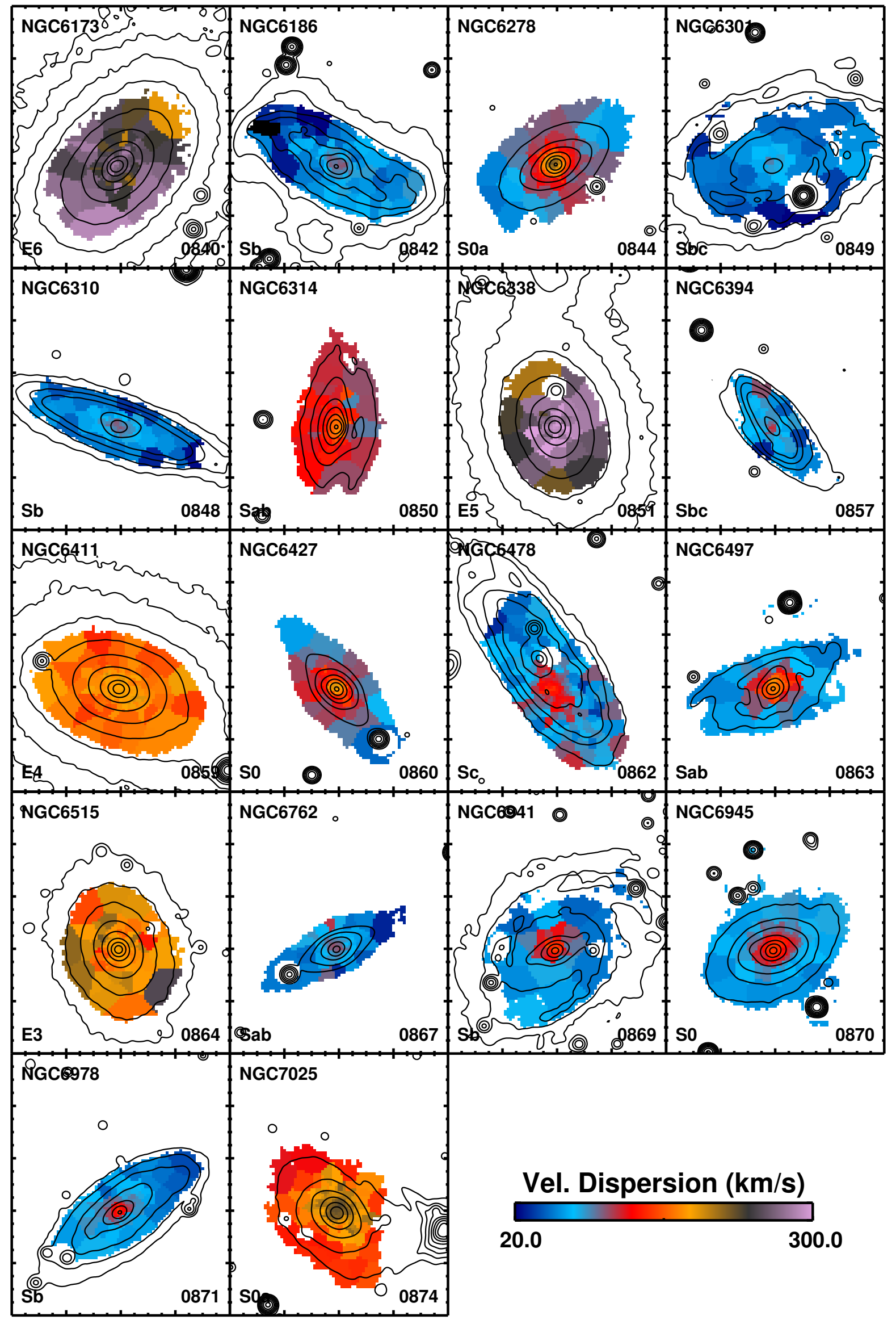

Fig. A.2. continued. 


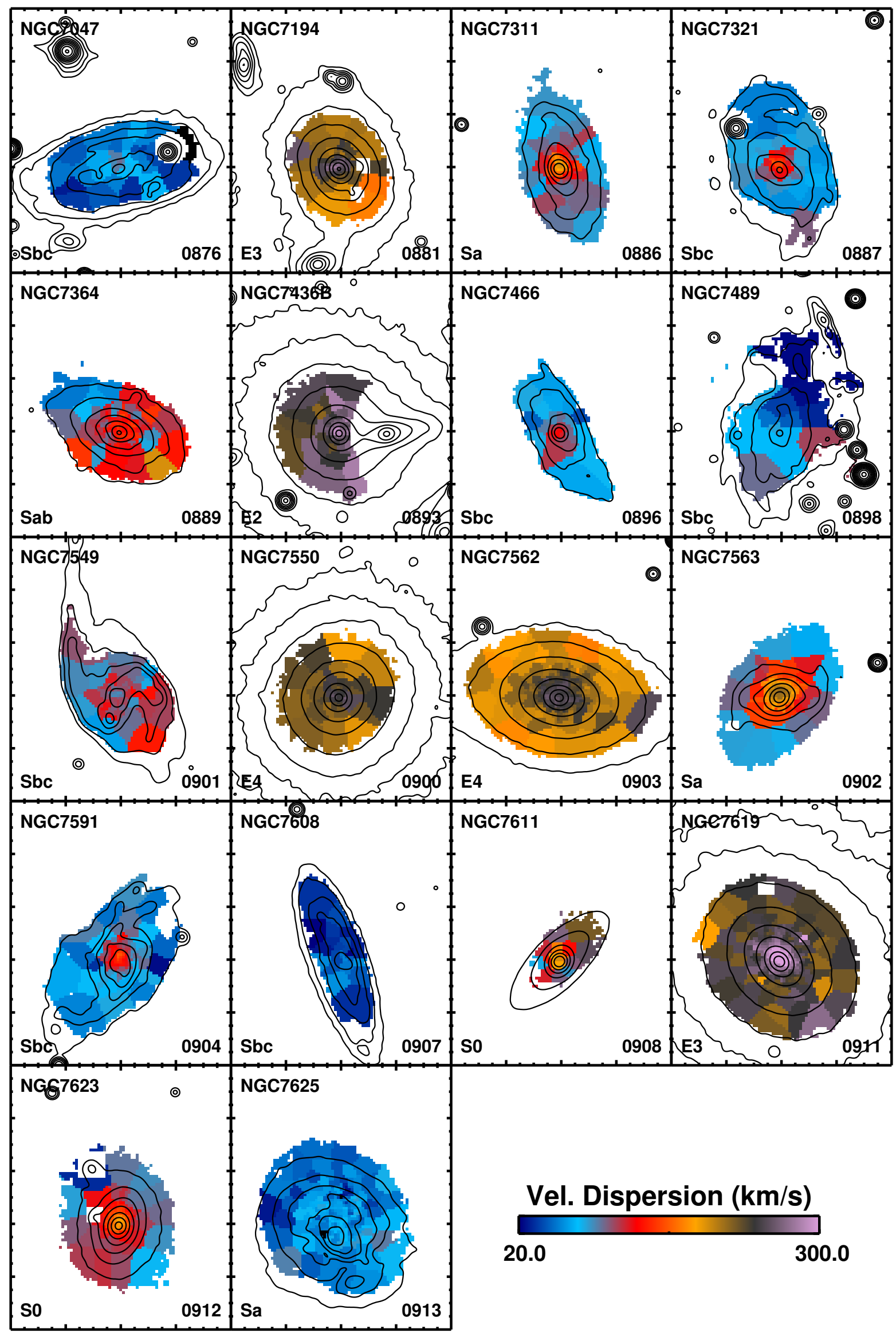

Fig. A.2. continued. 
J. Falcón-Barroso et al.: Stellar kinematics across the Hubble sequence

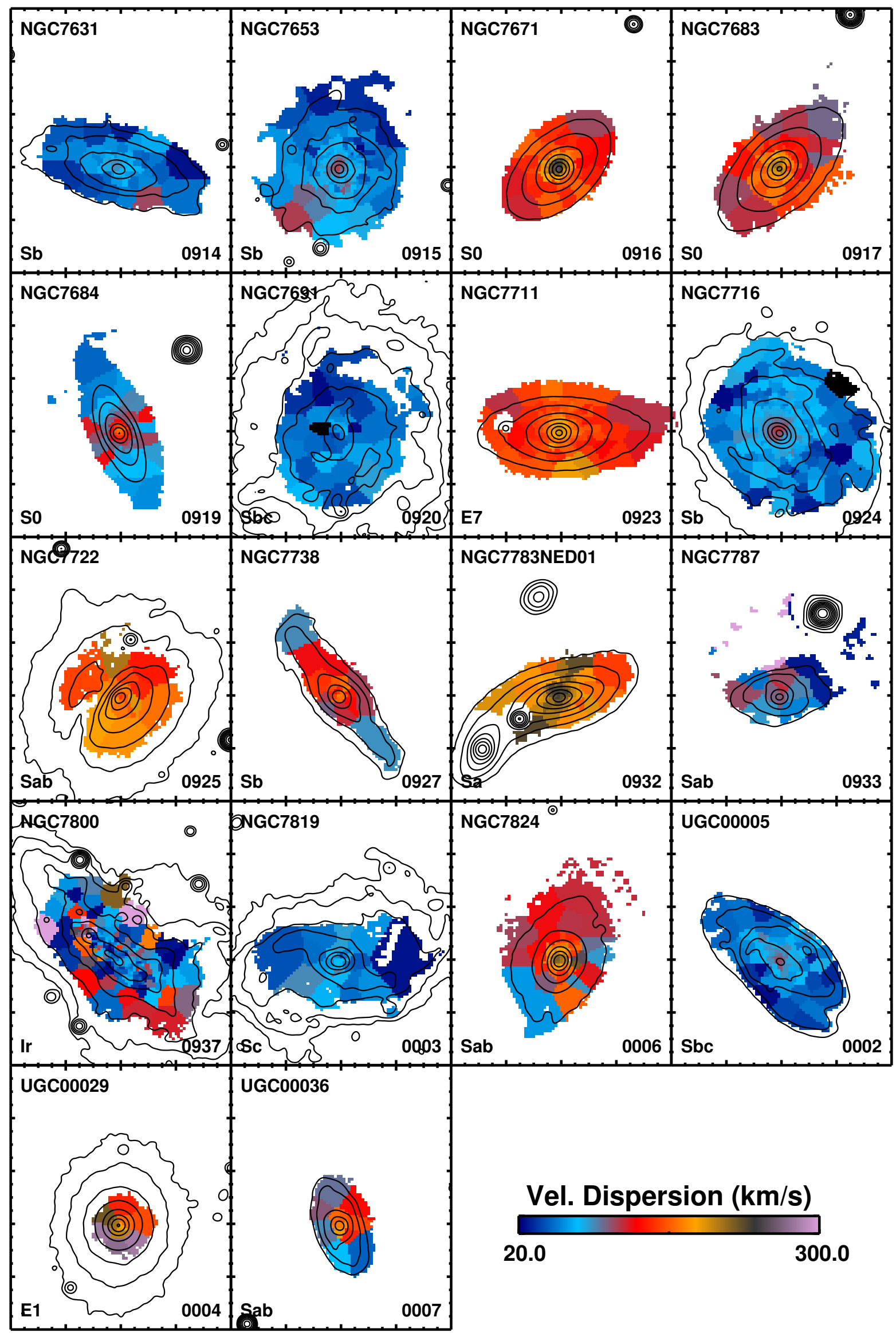

Fig. A.2. continued. 


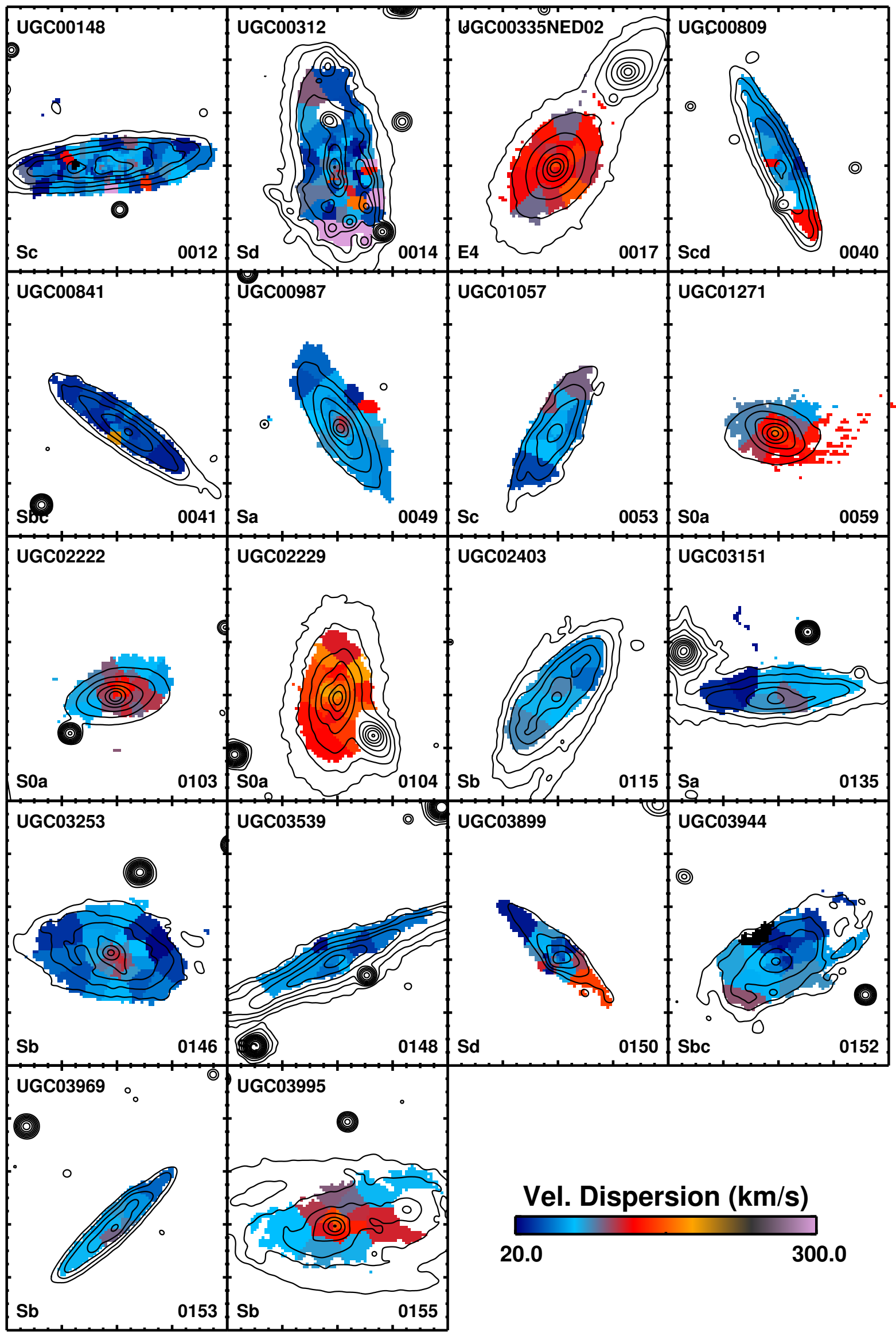

Fig. A.2. continued. 
J. Falcón-Barroso et al.: Stellar kinematics across the Hubble sequence

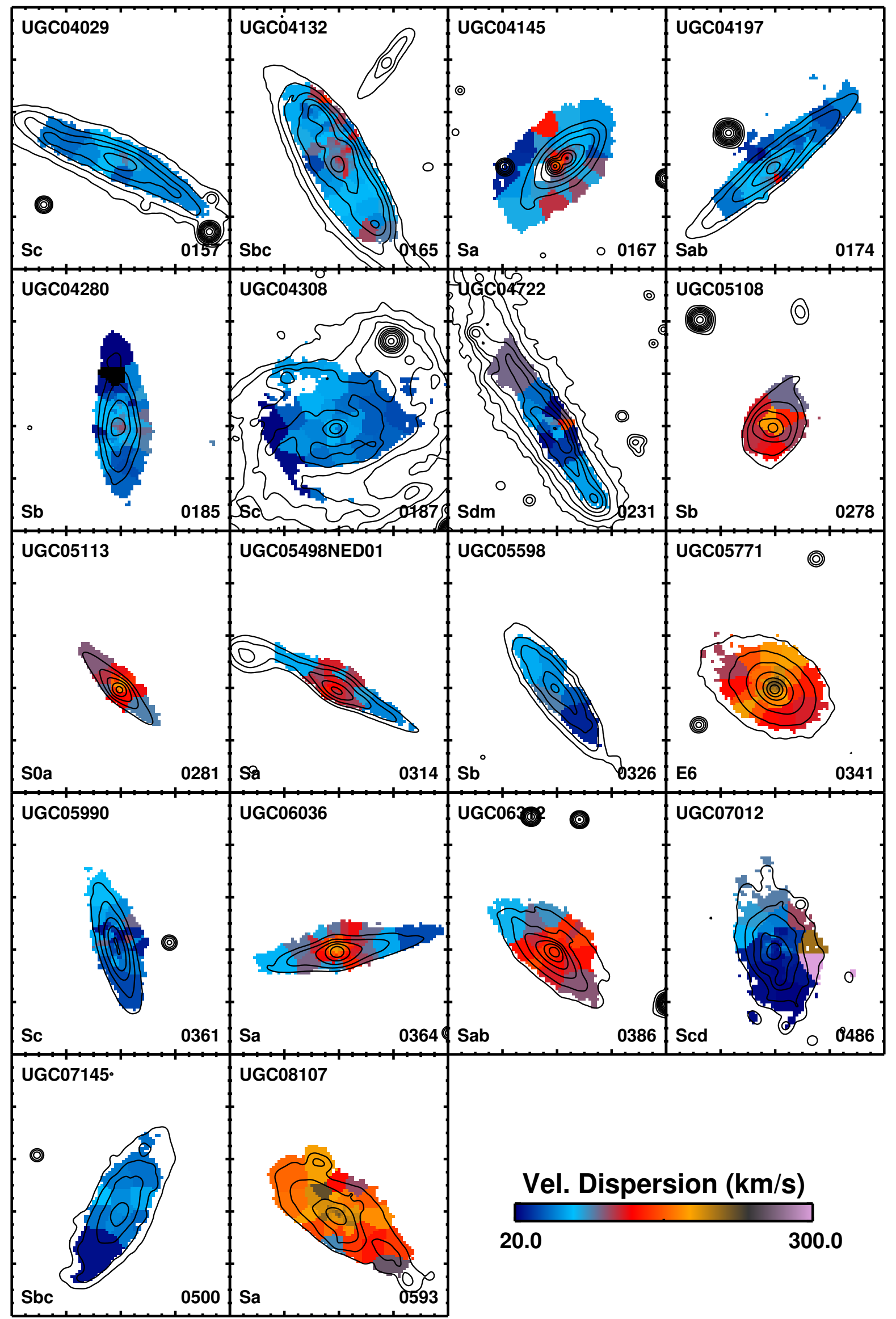

Fig. A.2. continued. 


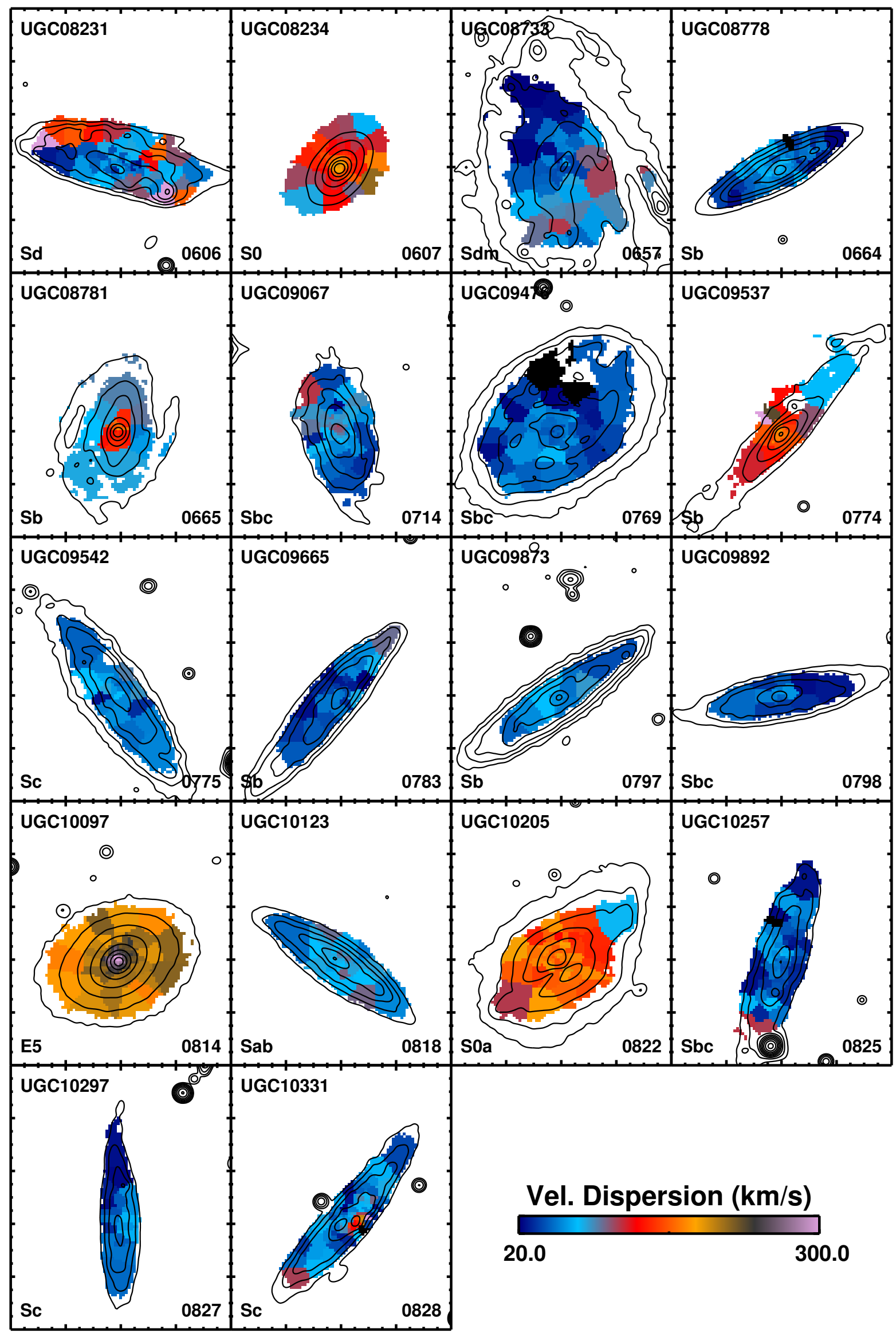

Fig. A.2. continued. 
J. Falcón-Barroso et al.: Stellar kinematics across the Hubble sequence

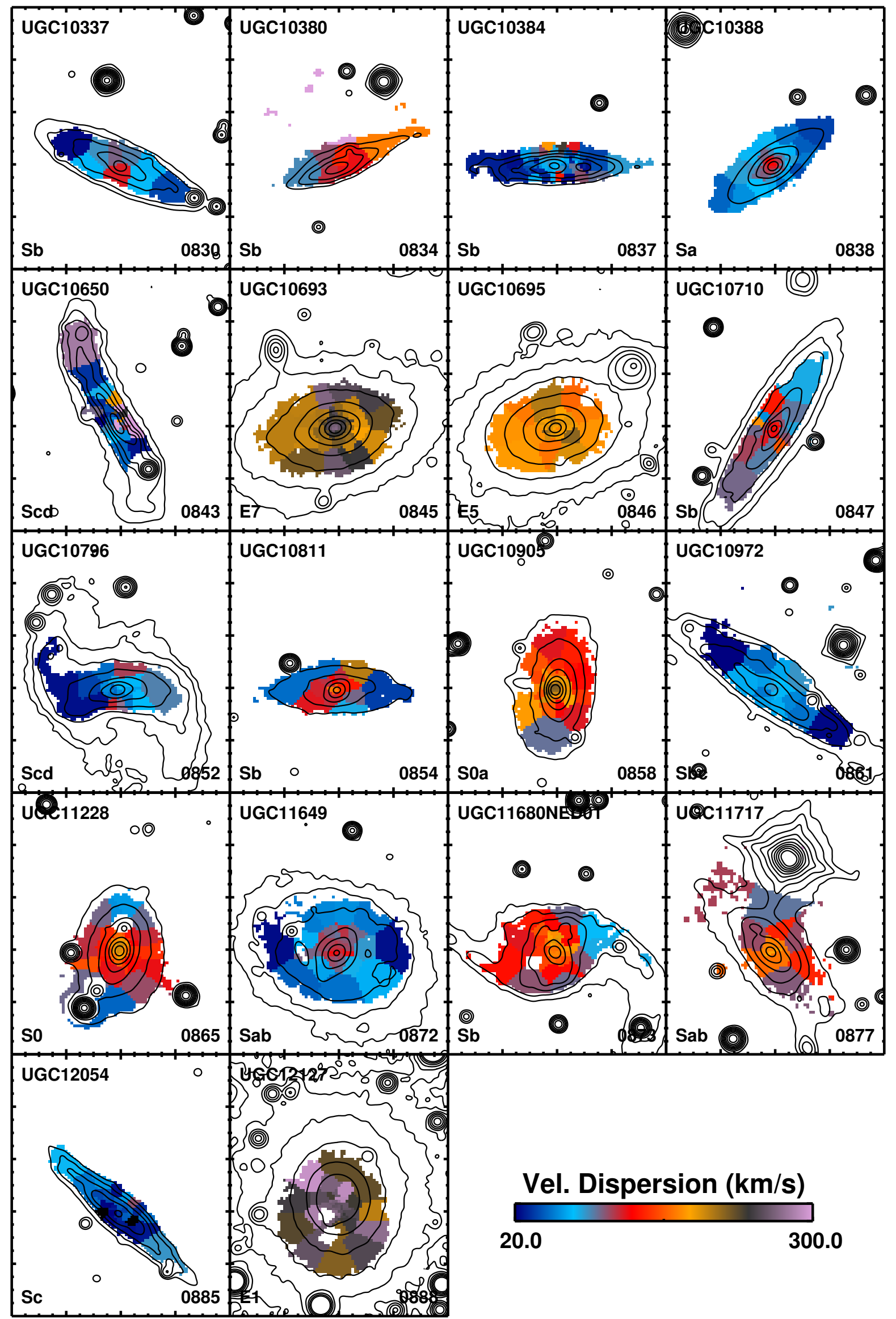

Fig. A.2. continued. 


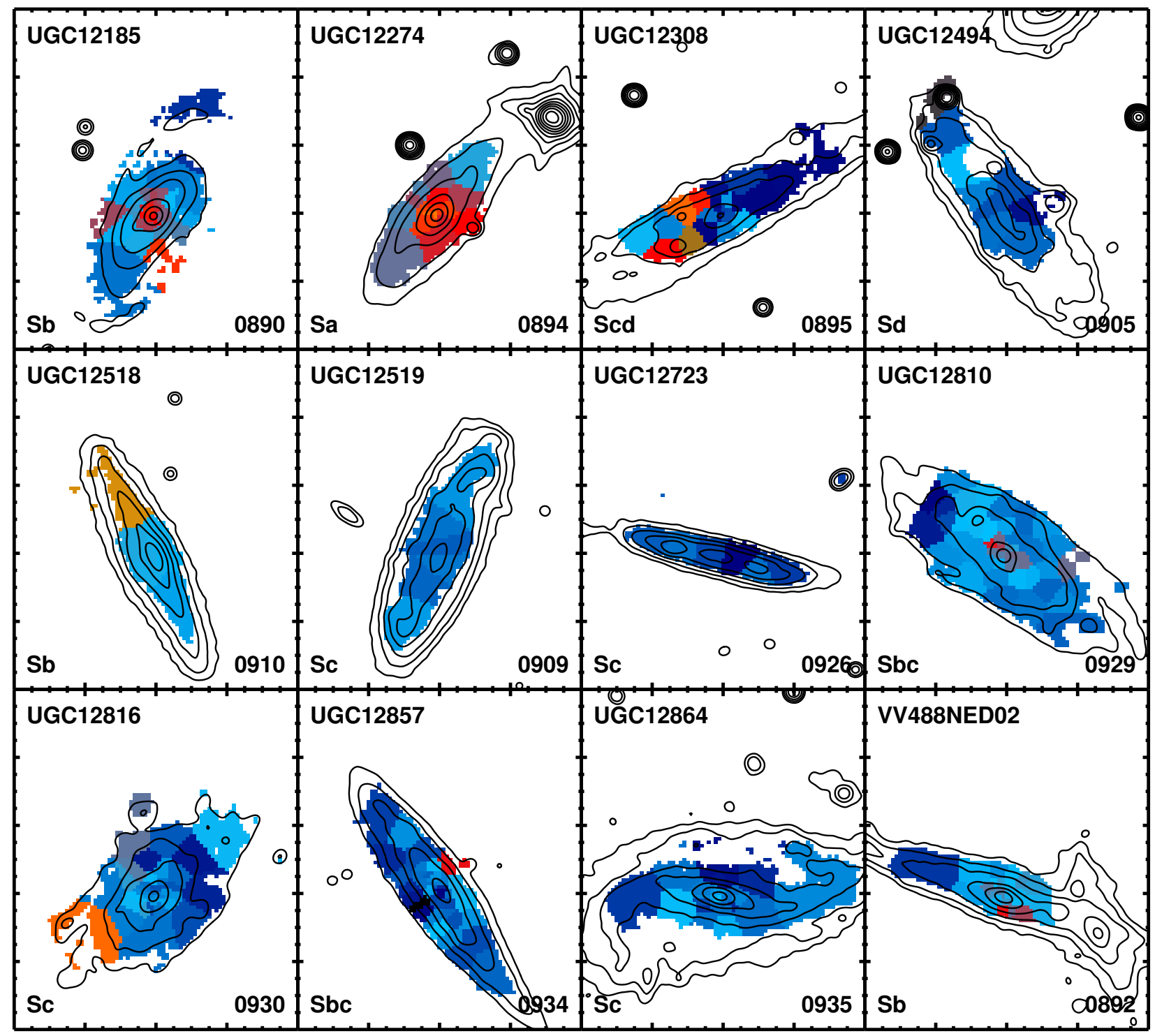

\section{Vel. Dispersion (km/s)}




\section{Appendix B: Additional table}

Table B.1. Basic properties of the CALIFA stellar kinematics sample.

\begin{tabular}{|c|c|c|c|c|c|c|c|c|c|}
\hline Galname & ID & $z$ & $\begin{array}{c}\text { PA } \\
\text { (deg) }\end{array}$ & $\epsilon$ & Type & $\begin{array}{c}M_{*} \\
\left(10^{10} M_{\odot}\right)\end{array}$ & $\begin{array}{c}M_{r} \\
(\mathrm{mag})\end{array}$ & $\begin{array}{c}R_{\mathrm{eff}} \\
(\operatorname{arcsec})\end{array}$ & $\begin{array}{c}R_{\max } \\
(\operatorname{arcsec})\end{array}$ \\
\hline IC 0480 & 159 & 0.015 & 167 & 0.77 & Sc & 1.41 & -20.43 & 24 & 38 \\
\hline IC 0540 & 274 & 0.007 & 170 & 0.63 & $\mathrm{Sab}$ & 0.75 & -19.27 & 14 & 27 \\
\hline IC 0674 & 381 & 0.025 & 117 & 0.63 & Sab & 8.09 & -22.07 & 9 & 36 \\
\hline IC 0944 & 663 & 0.023 & 105 & 0.65 & $\mathrm{Sab}$ & 18.20 & -22.37 & 19 & 37 \\
\hline IC 1079 & 781 & 0.029 & 82 & 0.51 & E4 & 21.23 & -23.21 & 37 & 26 \\
\hline IC 1151 & 817 & 0.007 & 28 & 0.63 & Scd & 0.70 & -20.26 & 22 & 37 \\
\hline IC 1199 & 824 & 0.016 & 157 & 0.57 & $\mathrm{Sb}$ & 4.67 & -21.46 & 20 & 28 \\
\hline IC 1256 & 856 & 0.016 & 89 & 0.36 & $\mathrm{Sb}$ & 2.00 & -21.18 & 17 & 25 \\
\hline IC 1528 & 005 & 0.013 & 75 & 0.56 & Sbc & 1.39 & -20.95 & 23 & 41 \\
\hline IC 1652 & 037 & 0.017 & 171 & 0.72 & SOa & 4.09 & -21.20 & 11 & 26 \\
\hline IC 1683 & 043 & 0.016 & 15 & 0.35 & $\mathrm{Sb}$ & 3.88 & -21.11 & 13 & 26 \\
\hline IC 1755 & 070 & 0.026 & 155 & 0.75 & $\mathrm{Sb}$ & 8.43 & -21.69 & 11 & 31 \\
\hline IC 2101 & 144 & 0.015 & 144 & 0.72 & Scd & 1.74 & -20.81 & 25 & 44 \\
\hline IC 2247 & 186 & 0.014 & 148 & 0.79 & $\mathrm{Sab}$ & 3.24 & -20.75 & 21 & 41 \\
\hline IC 2487 & 273 & 0.015 & 162 & 0.79 & $\mathrm{Sc}$ & 2.48 & -21.05 & 25 & 40 \\
\hline IC 4566 & 807 & 0.019 & 161 & 0.41 & $\mathrm{Sb}$ & 8.99 & -21.96 & 15 & 27 \\
\hline IC 5309 & 906 & 0.014 & 25 & 0.55 & Sc & 1.89 & -20.61 & 17 & 24 \\
\hline IC 5376 & 001 & 0.017 & 3 & 0.69 & $\mathrm{Sb}$ & 4.52 & -21.10 & 16 & 36 \\
\hline MCG-01-54-016 & 878 & 0.010 & 32 & 0.78 & Scd & 0.11 & -18.77 & 24 & 38 \\
\hline MCG-02-02-030 & 013 & 0.012 & 171 & 0.56 & $\mathrm{Sb}$ & 2.34 & -20.88 & 19 & 38 \\
\hline MCG-02-02-040 & 016 & 0.012 & 53 & 0.47 & Scd & 0.99 & -20.19 & 20 & 34 \\
\hline MCG-02-03-015 & 032 & 0.019 & 22 & 0.74 & $\mathrm{Sab}$ & 4.24 & -21.41 & 12 & 37 \\
\hline MCG-02-51-004 & 868 & 0.019 & 159 & 0.64 & $\mathrm{Sb}$ & 4.79 & -21.69 & 17 & 34 \\
\hline NGC 0001 & 008 & 0.015 & 107 & 0.32 & Sbc & 6.31 & -21.73 & 12 & 30 \\
\hline NGC 0023 & 009 & 0.015 & 177 & 0.30 & $\mathrm{Sb}$ & 10.96 & -22.47 & 17 & 26 \\
\hline NGC 0036 & 010 & 0.020 & 24 & 0.48 & $\mathrm{Sb}$ & 7.87 & -22.33 & 21 & 33 \\
\hline NGC 0155 & 018 & 0.021 & 167 & 0.14 & E1 & 15.00 & -22.41 & 15 & 25 \\
\hline NGC 0160 & 020 & 0.018 & 49 & 0.47 & $\mathrm{Sa}$ & 10.72 & -22.18 & 22 & 35 \\
\hline NGC 0169 & 022 & 0.015 & 90 & 0.47 & $\mathrm{Sab}$ & 39.90 & -21.87 & 34 & 34 \\
\hline NGC 0171 & 023 & 0.013 & 32 & 0.05 & $\mathrm{Sb}$ & 5.26 & -21.84 & 26 & 32 \\
\hline NGC 0177 & 024 & 0.013 & 8 & 0.42 & $\mathrm{Sab}$ & 2.34 & -20.70 & 13 & 37 \\
\hline NGC 0180 & 025 & 0.018 & 167 & 0.34 & $\mathrm{Sb}$ & 8.36 & -22.31 & 28 & 41 \\
\hline NGC 0192 & 026 & 0.014 & 168 & 0.57 & $\mathrm{Sab}$ & 7.05 & -21.59 & 22 & 39 \\
\hline NGC 0214 & 028 & 0.015 & 50 & 0.26 & $\mathrm{Sbc}$ & 6.65 & -22.16 & 18 & 31 \\
\hline NGC 0216 & 027 & 0.005 & 25 & 0.71 & $\mathrm{Sd}$ & 0.19 & -18.99 & 20 & 35 \\
\hline NGC 0217 & 029 & 0.013 & 112 & 0.74 & $\mathrm{Sa}$ & 12.50 & -21.90 & 23 & 41 \\
\hline NGC 0234 & 031 & 0.015 & 64 & 0.20 & $\mathrm{Sc}$ & 4.50 & -21.91 & 20 & 34 \\
\hline NGC 0237 & 030 & 0.014 & 175 & 0.32 & $\mathrm{Sc}$ & 2.04 & -21.14 & 15 & 35 \\
\hline NGC 0257 & 033 & 0.018 & 88 & 0.36 & $\mathrm{Sc}$ & 6.22 & -22.15 & 21 & 40 \\
\hline NGC 0364 & 035 & 0.017 & 35 & 0.28 & E7 & 9.16 & -21.56 & 15 & 24 \\
\hline NGC 0429 & 036 & 0.019 & 15 & 0.78 & $\mathrm{Sa}$ & 6.22 & -21.26 & 6 & 29 \\
\hline NGC 0444 & 039 & 0.016 & 158 & 0.74 & Scd & 0.74 & -20.23 & 23 & 32 \\
\hline NGC 0447 & 038 & 0.019 & 74 & 0.13 & $\mathrm{Sa}$ & 13.52 & -22.40 & 28 & 31 \\
\hline NGC 0477 & 042 & 0.020 & 128 & 0.50 & Sbc & 3.14 & -21.69 & 21 & 45 \\
\hline NGC 0496 & 045 & 0.020 & 32 & 0.46 & Scd & 2.59 & -21.40 & 19 & 35 \\
\hline NGC 0499 & 044 & 0.015 & 72 & 0.33 & E5 & 25.18 & -22.48 & 21 & 31 \\
\hline NGC 0504 & 046 & 0.014 & 44 & 0.60 & S0 & 2.95 & -20.76 & 8 & 30 \\
\hline NGC 0517 & 047 & 0.014 & 24 & 0.49 & So & 6.64 & -21.35 & 10 & 34 \\
\hline NGC 0528 & 050 & 0.016 & 57 & 0.52 & So & 7.48 & -21.68 & 12 & 28 \\
\hline NGC 0529 & 051 & 0.016 & 13 & 0.09 & E4 & 12.25 & -22.27 & 12 & 37 \\
\hline NGC 0551 & 052 & 0.017 & 137 & 0.56 & $\mathrm{Sbc}$ & 4.38 & -21.52 & 19 & 44 \\
\hline
\end{tabular}

Notes. Column 1: galaxy name. Column 2: CALIFA identification number for each galaxy. Column 3: redshift of the galaxy from SDSS (Abazajian et al. 2009). Column 4: position angle of the galaxy measured in the outer parts, using SDSS images. Column 5: average ellipticity measured in the outer parts of the galaxy, using SDSS images. Column 6: Hubble type of the galaxy from Walcher et al. (2014). Column 7: total stellar mass of the galaxy, measured as described in Walcher et al. (2014). Column 8: total absolute magnitude in $r$-band from SDSS (Abazajian et al. 2009). Column 9: effective radii (in arcsec) of the galaxy, measured as described in Walcher et al. (2014). Column 10: maximum radial extent of our kinematic maps (in arcsec). 
Table B.1. continued.

\begin{tabular}{|c|c|c|c|c|c|c|c|c|c|}
\hline Galname & ID & $z$ & $\begin{array}{c}\text { PA } \\
(\mathrm{deg})\end{array}$ & $\epsilon$ & Type & $\begin{array}{c}M_{*} \\
\left(10^{10} M_{\odot}\right)\end{array}$ & $\begin{array}{c}M_{r} \\
(\mathrm{mag})\end{array}$ & $\begin{array}{c}R_{\mathrm{eff}} \\
(\operatorname{arcsec})\end{array}$ & $\begin{array}{c}R_{\max } \\
(\operatorname{arcsec})\end{array}$ \\
\hline NGC 0681 & 061 & 0.006 & 65 & 0.33 & $\mathrm{Sa}$ & 3.10 & -20.71 & 30 & 37 \\
\hline NGC 0741 & 068 & 0.019 & 85 & 0.22 & E1 & 32.89 & -23.47 & 35 & 32 \\
\hline NGC 0755 & 069 & 0.005 & 49 & 0.61 & Scd & 0.24 & -19.43 & 28 & 39 \\
\hline NGC 0768 & 071 & 0.023 & 33 & 0.61 & Sc & 3.48 & -21.78 & 15 & 34 \\
\hline NGC 0774 & 072 & 0.015 & 164 & 0.18 & So & 8.39 & -21.55 & 12 & 26 \\
\hline NGC 0776 & 073 & 0.016 & 41 & 0.10 & $\mathrm{Sb}$ & 4.94 & -21.82 & 19 & 32 \\
\hline NGC 0781 & 074 & 0.012 & 11 & 0.70 & $\mathrm{Sa}$ & 2.96 & -20.80 & 8 & 32 \\
\hline NGC 0810 & 076 & 0.026 & 27 & 0.34 & E5 & 35.65 & -22.84 & 17 & 20 \\
\hline NGC 0825 & 077 & 0.011 & 50 & 0.51 & $\mathrm{Sa}$ & 2.64 & -20.70 & 12 & 34 \\
\hline NGC 0932 & 087 & 0.014 & 65 & 0.08 & SOa & 9.20 & -22.10 & 18 & 33 \\
\hline NGC 1056 & 100 & 0.005 & 153 & 0.32 & $\mathrm{Sa}$ & 1.05 & -19.94 & 14 & 37 \\
\hline NGC 1060 & 101 & 0.017 & 75 & 0.18 & E3 & 70.15 & -23.62 & 27 & 26 \\
\hline NGC 1093 & 108 & 0.018 & 99 & 0.39 & $\mathrm{Sbc}$ & 3.25 & -21.49 & 13 & 36 \\
\hline NGC 1167 & 119 & 0.016 & 62 & 0.23 & So & 49.20 & -22.98 & 24 & 30 \\
\hline NGC 1349 & 127 & 0.022 & 50 & 0.12 & E6 & 8.47 & -22.44 & 17 & 21 \\
\hline NGC 1542 & 131 & 0.012 & 131 & 0.59 & $\mathrm{Sab}$ & 2.74 & -20.74 & 15 & 23 \\
\hline NGC 1645 & 134 & 0.016 & 84 & 0.57 & SOa & 6.78 & -21.81 & 13 & 39 \\
\hline NGC 1677 & 143 & 0.009 & 137 & 0.71 & Scd & 0.38 & -19.46 & 12 & 29 \\
\hline NGC 2253 & 147 & 0.012 & 109 & 0.32 & Sbc & 3.34 & -21.55 & 15 & 36 \\
\hline NGC 2347 & 149 & 0.015 & 9 & 0.36 & Sbc & 8.71 & -22.12 & 18 & 42 \\
\hline NGC 2410 & 151 & 0.016 & 34 & 0.68 & $\mathrm{Sb}$ & 7.62 & -21.86 & 21 & 37 \\
\hline NGC 2449 & 156 & 0.016 & 135 & 0.52 & $\mathrm{Sab}$ & 7.28 & -21.68 & 16 & 33 \\
\hline NGC 2476 & 160 & 0.012 & 136 & 0.29 & E6 & 6.32 & -21.58 & 9 & 22 \\
\hline NGC 2480 & 161 & 0.008 & 167 & 0.43 & $\mathrm{Sdm}$ & 0.83 & -19.75 & 35 & 25 \\
\hline NGC 2481 & 162 & 0.007 & 6 & 0.16 & S0 & 4.83 & -20.80 & 9 & 34 \\
\hline NGC 2486 & 163 & 0.015 & 92 & 0.44 & $\mathrm{Sab}$ & 3.96 & -21.30 & 15 & 29 \\
\hline NGC 2487 & 164 & 0.016 & 132 & 0.15 & $\mathrm{Sb}$ & 5.90 & -22.19 & 28 & 35 \\
\hline NGC 2513 & 171 & 0.016 & 174 & 0.27 & $\mathrm{E} 2$ & 34.59 & -22.86 & 26 & 32 \\
\hline NGC 2540 & 183 & 0.021 & 131 & 0.39 & $\mathrm{Sbc}$ & 3.32 & -21.62 & 14 & 33 \\
\hline NGC 2553 & 188 & 0.016 & 67 & 0.50 & $\mathrm{Sb}$ & 6.89 & -21.30 & 9 & 19 \\
\hline NGC 2554 & 189 & 0.014 & 160 & 0.19 & SOa & 16.33 & -22.59 & 19 & 38 \\
\hline NGC 2592 & 201 & 0.007 & 45 & 0.22 & $\mathrm{E} 4$ & 4.15 & -20.72 & 9 & 28 \\
\hline NGC 2604 & 209 & 0.007 & 48 & 0.12 & $\mathrm{Sd}$ & 0.46 & -20.24 & 26 & 36 \\
\hline NGC 2639 & 219 & 0.011 & 130 & 0.35 & $\mathrm{Sa}$ & 14.72 & -22.33 & 17 & 38 \\
\hline NGC 2730 & 232 & 0.013 & 80 & 0.12 & Scd & 1.31 & -20.94 & 24 & 39 \\
\hline NGC 2880 & 272 & 0.005 & 142 & 0.36 & E7 & 4.69 & -21.10 & 18 & 36 \\
\hline NGC 2906 & 275 & 0.007 & 82 & 0.44 & $\mathrm{Sbc}$ & 2.46 & -20.79 & 19 & 33 \\
\hline NGC 2916 & 277 & 0.012 & 19 & 0.36 & $\mathrm{Sbc}$ & 5.66 & -22.09 & 26 & 40 \\
\hline NGC 2918 & 279 & 0.023 & 75 & 0.31 & E6 & 27.73 & -22.78 & 12 & 28 \\
\hline NGC 3057 & 312 & 0.005 & 23 & 0.27 & $\mathrm{Sdm}$ & 0.12 & -19.17 & 32 & 34 \\
\hline NGC 3106 & 311 & 0.021 & 116 & 0.10 & $\mathrm{Sab}$ & 16.29 & -22.79 & 21 & 32 \\
\hline NGC 3158 & 318 & 0.023 & 165 & 0.19 & E3 & 54.70 & -23.70 & 32 & 32 \\
\hline NGC 3160 & 319 & 0.023 & 140 & 0.76 & Sab & 8.99 & -21.51 & 15 & 36 \\
\hline NGC 3300 & 339 & 0.010 & 173 & 0.46 & SOa & 5.78 & -21.41 & 13 & 32 \\
\hline NGC 3303 & 340 & 0.020 & 159 & 0.51 & SOa & 11.51 & -22.33 & 15 & 21 \\
\hline NGC 3381 & 353 & 0.005 & 43 & 0.14 & $\mathrm{Sd}$ & 0.48 & -20.08 & 24 & 42 \\
\hline NGC 3615 & 387 & 0.022 & 42 & 0.42 & E5 & 24.15 & -22.98 & 15 & 18 \\
\hline NGC 3687 & 414 & 0.008 & 151 & 0.06 & $\mathrm{Sb}$ & 1.88 & -20.97 & 17 & 30 \\
\hline NGC 3811 & 436 & 0.010 & 171 & 0.23 & $\mathrm{Sbc}$ & 2.65 & -21.40 & 21 & 39 \\
\hline NGC 3815 & 437 & 0.012 & 67 & 0.50 & Sbc & 2.25 & -21.05 & 14 & 34 \\
\hline NGC 3994 & 476 & 0.010 & 8 & 0.49 & $\mathrm{Sbc}$ & 2.65 & -21.22 & 9 & 26 \\
\hline NGC 4003 & 479 & 0.022 & 168 & 0.28 & SOa & 11.83 & -22.00 & 14 & 22 \\
\hline NGC 4047 & 489 & 0.011 & 97 & 0.26 & $\mathrm{Sbc}$ & 4.86 & -21.90 & 16 & 33 \\
\hline NGC 4149 & 502 & 0.010 & 85 & 0.60 & $\mathrm{Sa}$ & 2.30 & -20.63 & 18 & 36 \\
\hline NGC 4185 & 515 & 0.013 & 164 & 0.33 & $\mathrm{Sbc}$ & 4.69 & -21.88 & 30 & 38 \\
\hline NGC 4210 & 518 & 0.009 & 97 & 0.24 & $\mathrm{Sb}$ & 1.93 & -20.98 & 21 & 36 \\
\hline NGC 4470 & 548 & 0.008 & 179 & 0.32 & $\mathrm{Sc}$ & 0.98 & -20.72 & 15 & 33 \\
\hline NGC 4644 & 569 & 0.016 & 57 & 0.71 & $\mathrm{Sb}$ & 2.82 & -21.03 & 12 & 29 \\
\hline NGC 4676A & 577 & 0.022 & 2 & 0.85 & $\mathrm{Sdm}$ & 6.50 & -22.17 & 38 & 31 \\
\hline
\end{tabular}


Table B.1. continued.

\begin{tabular}{|c|c|c|c|c|c|c|c|c|c|}
\hline Galname & ID & $z$ & $\begin{array}{c}\text { PA } \\
(\mathrm{deg})\end{array}$ & $\epsilon$ & Type & $\begin{array}{c}M_{*} \\
\left(10^{10} M_{\odot}\right)\end{array}$ & $\begin{array}{c}M_{r} \\
(\mathrm{mag})\end{array}$ & $\begin{array}{c}R_{\mathrm{eff}} \\
(\operatorname{arcsec})\end{array}$ & $\begin{array}{c}R_{\max } \\
(\operatorname{arcsec})\end{array}$ \\
\hline NGC 4676B & 2999 & 0.022 & 43 & 0.44 & S0 & 7.18 & -22.09 & 15 & 23 \\
\hline NGC 4711 & 580 & 0.014 & 41 & 0.47 & $\mathrm{Sbc}$ & 2.05 & -21.05 & 17 & 33 \\
\hline NGC 4816 & 588 & 0.023 & 80 & 0.31 & E1 & 32.06 & -23.03 & 30 & 30 \\
\hline NGC 4841A & 589 & 0.023 & 42 & 0.11 & E3 & 35.16 & -22.83 & 20 & 25 \\
\hline NGC 4874 & 592 & 0.024 & 46 & 0.23 & $\mathrm{E} 0$ & 49.54 & -24.11 & 55 & 29 \\
\hline NGC 4956 & 602 & 0.016 & 39 & 0.17 & E1 & 9.68 & -22.38 & 9 & 21 \\
\hline NGC 4961 & 603 & 0.009 & 100 & 0.31 & Scd & 0.48 & -20.25 & 15 & 33 \\
\hline NGC 5000 & 608 & 0.019 & 1 & 0.24 & Sbc & 5.37 & -21.81 & 16 & 30 \\
\hline NGC 5016 & 611 & 0.009 & 57 & 0.23 & $\mathrm{Sbc}$ & 1.72 & -21.06 & 17 & 35 \\
\hline NGC 5029 & 612 & 0.029 & 149 & 0.40 & E6 & 31.77 & -23.28 & 25 & 28 \\
\hline NGC 5056 & 614 & 0.019 & 3 & 0.44 & $\mathrm{Sc}$ & 3.02 & -21.82 & 15 & 38 \\
\hline NGC 5205 & 630 & 0.006 & 169 & 0.35 & Sbc & 0.73 & -20.12 & 19 & 41 \\
\hline NGC 5216 & 633 & 0.010 & 33 & 0.32 & E0 & 3.20 & -21.07 & 20 & 26 \\
\hline NGC 5218 & 634 & 0.010 & 101 & 0.14 & $\mathrm{Sab}$ & 4.49 & -21.43 & 18 & 36 \\
\hline NGC 5378 & 676 & 0.010 & 86 & 0.22 & $\mathrm{Sb}$ & 3.83 & -21.27 & 24 & 34 \\
\hline NGC 5406 & 684 & 0.018 & 111 & 0.29 & $\mathrm{Sb}$ & 18.75 & -22.57 & 20 & 40 \\
\hline NGC 5480 & 707 & 0.006 & 41 & 0.18 & Scd & 1.38 & -20.76 & 25 & 41 \\
\hline NGC 5485 & 708 & 0.006 & 174 & 0.32 & E5 & 10.57 & -21.95 & 31 & 38 \\
\hline NGC 5520 & 715 & 0.006 & 63 & 0.49 & Sbc & 0.73 & -20.18 & 12 & 34 \\
\hline NGC 5614 & 740 & 0.013 & 128 & 0.19 & $\mathrm{Sa}$ & 19.86 & -22.77 & 18 & 35 \\
\hline NGC 5630 & 749 & 0.009 & 93 & 0.70 & $\mathrm{Sdm}$ & 0.47 & -20.37 & 22 & 38 \\
\hline NGC 5631 & 744 & 0.007 & 30 & 0.06 & So & 8.47 & -21.74 & 19 & 34 \\
\hline NGC 5633 & 748 & 0.008 & 16 & 0.26 & $\mathrm{Sbc}$ & 1.82 & -20.94 & 13 & 35 \\
\hline NGC 5657 & 754 & 0.013 & 164 & 0.63 & $\mathrm{Sbc}$ & 1.92 & -20.98 & 10 & 39 \\
\hline NGC 5682 & 758 & 0.008 & 125 & 0.76 & Scd & 0.25 & -19.39 & 26 & 38 \\
\hline NGC 5720 & 764 & 0.026 & 131 & 0.44 & Sbc & 7.05 & -22.29 & 16 & 27 \\
\hline NGC 5732 & 768 & 0.013 & 43 & 0.48 & $\mathrm{Sbc}$ & 0.85 & -20.46 & 14 & 32 \\
\hline NGC 5784 & 778 & 0.018 & 19 & 0.13 & So & 16.44 & -22.61 & 13 & 29 \\
\hline NGC 5797 & 780 & 0.013 & 130 & 0.45 & E7 & 7.01 & -22.13 & 18 & 31 \\
\hline NGC 5876 & 787 & 0.011 & 51 & 0.59 & S0a & 7.96 & -21.41 & 12 & 31 \\
\hline NGC 5888 & 789 & 0.029 & 150 & 0.38 & $\mathrm{Sb}$ & 16.07 & -22.74 & 16 & 31 \\
\hline NGC 5908 & 791 & 0.011 & 154 & 0.36 & $\mathrm{Sa}$ & 16.71 & -22.17 & 34 & 42 \\
\hline NGC 5930 & 795 & 0.009 & 161 & 0.54 & $\mathrm{Sab}$ & 4.30 & -21.36 & 16 & 37 \\
\hline NGC 5934 & 796 & 0.019 & 24 & 0.59 & $\mathrm{Sb}$ & 8.75 & -21.80 & 13 & 36 \\
\hline NGC 5947 & 4034 & 0.020 & 61 & 0.15 & Sbc & 3.48 & -21.56 & 13 & 32 \\
\hline NGC 5953 & 801 & 0.007 & 43 & 0.10 & $\mathrm{Sa}$ & 3.01 & -21.09 & 10 & 34 \\
\hline NGC 5966 & 806 & 0.015 & 83 & 0.39 & E4 & 10.21 & -22.08 & 18 & 34 \\
\hline NGC 5971 & 804 & 0.011 & 132 & 0.56 & $\mathrm{Sb}$ & 2.07 & -20.80 & 12 & 27 \\
\hline NGC 5980 & 810 & 0.014 & 11 & 0.60 & Sbc & 5.25 & -21.81 & 17 & 40 \\
\hline NGC 5987 & 809 & 0.010 & 62 & 0.65 & $\mathrm{Sa}$ & 16.22 & -22.15 & 33 & 37 \\
\hline NGC 6004 & 813 & 0.013 & 91 & 0.20 & $\mathrm{Sbc}$ & 4.86 & -21.86 & 22 & 37 \\
\hline NGC 6020 & 815 & 0.014 & 133 & 0.31 & E4 & 10.02 & -22.08 & 19 & 25 \\
\hline NGC 6021 & 816 & 0.016 & 157 & 0.27 & E5 & 10.14 & -21.88 & 9 & 27 \\
\hline NGC 6032 & 820 & 0.014 & 0 & 0.38 & $\mathrm{Sbc}$ & 3.37 & -21.30 & 27 & 39 \\
\hline NGC 6060 & 821 & 0.015 & 102 & 0.57 & $\mathrm{Sb}$ & 8.59 & -22.23 & 28 & 35 \\
\hline NGC 6063 & 823 & 0.010 & 156 & 0.44 & $\mathrm{Sbc}$ & 1.38 & -20.55 & 20 & 36 \\
\hline NGC 6081 & 826 & 0.017 & 128 & 0.59 & S0a & 13.12 & -21.95 & 12 & 30 \\
\hline NGC 6125 & 829 & 0.015 & 4 & 0.04 & E1 & 24.21 & -22.86 & 21 & 28 \\
\hline NGC 6132 & 831 & 0.017 & 125 & 0.64 & Sbc & 1.63 & -21.04 & 14 & 31 \\
\hline NGC 6146 & 832 & 0.029 & 73 & 0.24 & E5 & 42.56 & -23.48 & 15 & 26 \\
\hline NGC 6150 & 835 & 0.029 & 58 & 0.45 & E7 & 26.67 & -22.65 & 11 & 29 \\
\hline NGC 6168 & 841 & 0.009 & 110 & 0.77 & Sc & 0.73 & -20.00 & 26 & 34 \\
\hline NGC 6173 & 840 & 0.029 & 144 & 0.37 & E6 & 53.09 & -23.85 & 38 & 32 \\
\hline NGC 6186 & 842 & 0.010 & 49 & 0.23 & $\mathrm{Sb}$ & 3.71 & -21.24 & 20 & 35 \\
\hline NGC 6278 & 844 & 0.009 & 126 & 0.42 & S0a & 8.30 & -21.49 & 11 & 33 \\
\hline NGC 6301 & 849 & 0.028 & 108 & 0.40 & $\mathrm{Sbc}$ & 10.42 & -22.76 & 24 & 39 \\
\hline NGC 6310 & 848 & 0.011 & 69 & 0.72 & $\mathrm{Sb}$ & 3.64 & -20.99 & 23 & 33 \\
\hline NGC 6314 & 850 & 0.022 & 173 & 0.47 & $\mathrm{Sab}$ & 16.26 & -22.46 & 12 & 37 \\
\hline NGC 6338 & 851 & 0.027 & 15 & 0.38 & E5 & 49.09 & -23.48 & 28 & 26 \\
\hline
\end{tabular}


Table B.1. continued.

\begin{tabular}{|c|c|c|c|c|c|c|c|c|c|}
\hline Galname & ID & $z$ & $\begin{array}{c}\text { PA } \\
(\operatorname{deg})\end{array}$ & $\epsilon$ & Type & $\begin{array}{c}M_{*} \\
\left(10^{10} M_{\odot}\right)\end{array}$ & $\begin{array}{c}M_{r} \\
(\mathrm{mag})\end{array}$ & $\begin{array}{c}R_{\mathrm{eff}} \\
(\operatorname{arcsec})\end{array}$ & $\begin{array}{c}R_{\max } \\
(\operatorname{arcsec})\end{array}$ \\
\hline NGC 6394 & 857 & 0.028 & 42 & 0.64 & Sbc & 7.87 & -21.87 & 14 & 24 \\
\hline NGC 6411 & 859 & 0.012 & 65 & 0.35 & $\mathrm{E} 4$ & 12.08 & -22.42 & 34 & 33 \\
\hline NGC 6427 & 860 & 0.011 & 34 & 0.57 & So & 5.64 & -21.37 & 8 & 34 \\
\hline NGC 6478 & 862 & 0.023 & 34 & 0.63 & Sc & 10.33 & -22.57 & 23 & 38 \\
\hline NGC 6497 & 863 & 0.010 & 112 & 0.51 & $\mathrm{Sab}$ & 10.89 & -22.09 & 13 & 34 \\
\hline NGC 6515 & 864 & 0.023 & 12 & 0.35 & E3 & 15.60 & -22.73 & 19 & 28 \\
\hline NGC 6762 & 867 & 0.010 & 119 & 0.72 & $\mathrm{Sab}$ & 2.42 & -20.46 & 9 & 31 \\
\hline NGC 6941 & 869 & 0.021 & 131 & 0.26 & $\mathrm{Sb}$ & 8.77 & -22.39 & 20 & 32 \\
\hline NGC 6945 & 870 & 0.013 & 127 & 0.36 & S0 & 24.49 & -21.91 & 13 & 31 \\
\hline NGC 6978 & 871 & 0.020 & 126 & 0.57 & $\mathrm{Sb}$ & 10.79 & -22.15 & 18 & 34 \\
\hline NGC 7025 & 874 & 0.017 & 39 & 0.32 & SOa & 33.65 & -22.73 & 13 & 31 \\
\hline NGC 7047 & 876 & 0.019 & 107 & 0.45 & Sbc & 6.18 & -21.83 & 18 & 29 \\
\hline NGC 7194 & 881 & 0.027 & 18 & 0.30 & E3 & 27.86 & -23.05 & 17 & 22 \\
\hline NGC 7311 & 886 & 0.015 & 9 & 0.47 & $\mathrm{Sa}$ & 11.72 & -22.45 & 12 & 37 \\
\hline NGC 7321 & 887 & 0.024 & 14 & 0.32 & $\mathrm{Sbc}$ & 8.53 & -22.48 & 15 & 32 \\
\hline NGC 7364 & 889 & 0.016 & 65 & 0.32 & $\mathrm{Sab}$ & 7.62 & -22.04 & 12 & 32 \\
\hline NGC 7436B & 893 & 0.025 & 41 & 0.15 & E2 & 82.04 & -23.50 & 27 & 27 \\
\hline NGC 7466 & 896 & 0.025 & 25 & 0.62 & Sbc & 5.60 & -21.86 & 13 & 31 \\
\hline NGC 7489 & 898 & 0.021 & 160 & 0.47 & $\mathrm{Sbc}$ & 3.17 & -22.07 & 20 & 39 \\
\hline NGC 7549 & 901 & 0.016 & 16 & 0.60 & $\mathrm{Sbc}$ & 3.97 & -21.75 & 20 & 34 \\
\hline NGC 7550 & 900 & 0.017 & 154 & 0.09 & E4 & 27.04 & -22.89 & 24 & 25 \\
\hline NGC 7562 & 903 & 0.012 & 83 & 0.32 & E4 & 17.66 & -22.54 & 20 & 36 \\
\hline NGC 7563 & 902 & 0.014 & 149 & 0.47 & $\mathrm{Sa}$ & 9.18 & -21.54 & 9 & 31 \\
\hline NGC 7591 & 904 & 0.017 & 150 & 0.46 & $\mathrm{Sbc}$ & 5.75 & -21.91 & 16 & 33 \\
\hline NGC 7608 & 907 & 0.012 & 18 & 0.73 & $\mathrm{Sbc}$ & 1.24 & -20.00 & 20 & 33 \\
\hline NGC 7611 & 908 & 0.011 & 134 & 0.55 & S0 & 7.93 & -21.32 & 11 & 21 \\
\hline NGC 7619 & 911 & 0.013 & 50 & 0.17 & E3 & 8.79 & -22.69 & 35 & 34 \\
\hline NGC 7623 & 912 & 0.012 & 7 & 0.30 & So & 9.57 & -21.47 & 10 & 31 \\
\hline NGC 7625 & 913 & 0.005 & 10 & 0.04 & $\mathrm{Sa}$ & 1.33 & -20.26 & 14 & 34 \\
\hline NGC 7631 & 914 & 0.013 & 76 & 0.62 & $\mathrm{Sb}$ & 3.38 & -21.10 & 17 & 33 \\
\hline NGC 7653 & 915 & 0.014 & -11 & 0.18 & $\mathrm{Sb}$ & 3.16 & -21.58 & 12 & 38 \\
\hline NGC 7671 & 916 & 0.013 & 133 & 0.37 & So & 9.04 & -21.76 & 11 & 26 \\
\hline NGC 7683 & 917 & 0.012 & 138 & 0.48 & So & 10.45 & -21.74 & 14 & 33 \\
\hline NGC 7684 & 919 & 0.017 & 22 & 0.66 & So & 9.68 & -21.69 & 9 & 38 \\
\hline NGC 7691 & 920 & 0.013 & 171 & 0.21 & $\mathrm{Sbc}$ & 1.64 & -21.34 & 28 & 34 \\
\hline NGC 7711 & 923 & 0.014 & 92 & 0.55 & E7 & 11.30 & -22.02 & 15 & 42 \\
\hline NGC 7716 & 924 & 0.009 & 31 & 0.19 & $\mathrm{Sb}$ & 2.45 & -21.04 & 21 & 38 \\
\hline NGC 7722 & 925 & 0.013 & 148 & 0.27 & $\mathrm{Sab}$ & 17.58 & -22.05 & 21 & 24 \\
\hline NGC 7738 & 927 & 0.023 & 34 & 0.59 & $\mathrm{Sb}$ & 12.00 & -22.23 & 14 & 37 \\
\hline NGC 7783NED01 & 932 & 0.026 & 120 & 0.54 & $\mathrm{Sa}$ & 28.51 & -22.59 & 15 & 31 \\
\hline NGC 7787 & 933 & 0.022 & 104 & 0.71 & $\mathrm{Sab}$ & 4.18 & -21.17 & 11 & 23 \\
\hline NGC 7800 & 937 & 0.006 & 44 & 0.61 & $\mathrm{Ir}$ & 0.19 & -19.56 & 32 & 37 \\
\hline NGC 7819 & 003 & 0.017 & 105 & 0.41 & $\mathrm{Sc}$ & 2.45 & -21.06 & 23 & 37 \\
\hline NGC 7824 & 006 & 0.020 & 143 & 0.37 & $\mathrm{Sab}$ & 17.62 & -22.26 & 11 & 38 \\
\hline UGC 00005 & 002 & 0.024 & 44 & 0.53 & $\mathrm{Sbc}$ & 6.78 & -22.09 & 16 & 33 \\
\hline UGC 00029 & 004 & 0.029 & 173 & 0.30 & E1 & 10.86 & -22.66 & 17 & 13 \\
\hline UGC 00036 & 007 & 0.021 & 18 & 0.61 & $\mathrm{Sab}$ & 10.05 & -21.69 & 10 & 20 \\
\hline UGC 00148 & 012 & 0.014 & 96 & 0.75 & $\mathrm{Sc}$ & 1.29 & -20.75 & 20 & 36 \\
\hline UGC 00312 & 014 & 0.014 & 7 & 0.46 & $\mathrm{Sd}$ & 0.60 & -20.69 & 20 & 38 \\
\hline UGC 00335NED02 & 017 & 0.018 & 149 & 0.49 & $\mathrm{E} 4$ & 6.07 & -21.39 & 18 & 24 \\
\hline UGC 00809 & 040 & 0.014 & 23 & 0.81 & Scd & 0.49 & -19.72 & 20 & 36 \\
\hline UGC 00841 & 041 & 0.019 & 54 & 0.77 & $\mathrm{Sbc}$ & 1.03 & -20.26 & 17 & 31 \\
\hline UGC 00987 & 049 & 0.016 & 30 & 0.64 & $\mathrm{Sa}$ & 4.11 & -21.21 & 12 & 34 \\
\hline UGC 01057 & 053 & 0.021 & 152 & 0.69 & $\mathrm{Sc}$ & 1.27 & -20.81 & 14 & 27 \\
\hline UGC 01271 & 059 & 0.017 & 99 & 0.47 & SOa & 6.71 & -21.42 & 9 & 29 \\
\hline UGC 02222 & 103 & 0.017 & 96 & 0.57 & $\mathrm{~S} 0 \mathrm{a}$ & 5.53 & -21.42 & 10 & 23 \\
\hline UGC 02229 & 104 & 0.024 & 177 & 0.52 & SOa & 7.76 & -22.03 & 19 & 25 \\
\hline UGC 02403 & 115 & 0.014 & 153 & 0.59 & $\mathrm{Sb}$ & 3.19 & -20.80 & 19 & 26 \\
\hline UGC 03151 & 135 & 0.015 & 93 & 0.73 & $\mathrm{Sa}$ & 5.71 & -21.41 & 20 & 30 \\
\hline
\end{tabular}


Table B.1. continued.

\begin{tabular}{|c|c|c|c|c|c|c|c|c|c|}
\hline Galname & ID & $z$ & $\begin{array}{c}\text { PA } \\
(\operatorname{deg})\end{array}$ & $\epsilon$ & Type & $\begin{array}{c}M_{*} \\
\left(10^{10} M_{\odot}\right)\end{array}$ & $\begin{array}{c}M_{r} \\
(\mathrm{mag})\end{array}$ & $\begin{array}{c}R_{\mathrm{eff}} \\
(\operatorname{arcsec})\end{array}$ & $\begin{array}{c}R_{\max } \\
(\operatorname{arcsec})\end{array}$ \\
\hline UGC 03253 & 146 & 0.014 & 87 & 0.47 & $\mathrm{Sb}$ & 2.69 & -21.16 & 15 & 33 \\
\hline UGC 03539 & 148 & 0.011 & 117 & 0.69 & $\mathrm{Sc}$ & 0.70 & -19.69 & 20 & 38 \\
\hline UGC 03899 & 150 & 0.013 & 44 & 0.70 & $\mathrm{Sd}$ & 0.17 & -19.23 & 9 & 30 \\
\hline UGC 03944 & 152 & 0.013 & 120 & 0.57 & $\mathrm{Sbc}$ & 0.99 & -20.42 & 17 & 33 \\
\hline UGC 03969 & 153 & 0.027 & 134 & 0.78 & $\mathrm{Sb}$ & 4.78 & -21.19 & 15 & 29 \\
\hline UGC 03995 & 155 & 0.016 & 90 & 0.56 & $\mathrm{Sb}$ & 8.36 & -22.12 & 25 & 39 \\
\hline UGC 04029 & 157 & 0.015 & 63 & 0.79 & Sc & 2.14 & -20.75 & 26 & 37 \\
\hline UGC 04132 & 165 & 0.017 & 27 & 0.69 & $\mathrm{Sbc}$ & 5.82 & -21.81 & 22 & 35 \\
\hline UGC 04145 & 167 & 0.016 & 138 & 0.53 & $\mathrm{Sa}$ & 9.10 & -21.43 & 9 & 29 \\
\hline UGC 04197 & 174 & 0.015 & 130 & 0.79 & $\mathrm{Sab}$ & 5.15 & -20.92 & 18 & 41 \\
\hline UGC 04280 & 185 & 0.012 & 3 & 0.68 & $\mathrm{Sb}$ & 1.37 & -20.29 & 11 & 36 \\
\hline UGC 04308 & 187 & 0.012 & 113 & 0.14 & Sc & 1.84 & -21.29 & 24 & 33 \\
\hline UGC 04722 & 231 & 0.006 & 31 & 0.79 & $\mathrm{Sdm}$ & 0.05 & -18.18 & 32 & 38 \\
\hline UGC 05108 & 278 & 0.027 & 138 & 0.60 & $\mathrm{Sb}$ & 7.74 & -22.12 & 9 & 19 \\
\hline UGC 05113 & 281 & 0.023 & 41 & 0.74 & SOa & 12.62 & -21.76 & 8 & 22 \\
\hline UGC 05498NED01 & 314 & 0.021 & 61 & 0.79 & $\mathrm{Sa}$ & 6.38 & -21.36 & 13 & 31 \\
\hline UGC 05598 & 326 & 0.019 & 35 & 0.74 & $\mathrm{Sb}$ & 1.71 & -20.75 & 15 & 27 \\
\hline UGC 05771 & 341 & 0.025 & 60 & 0.33 & E6 & 20.75 & -22.35 & 12 & 27 \\
\hline UGC 05990 & 361 & 0.005 & 15 & 0.74 & Sc & 0.16 & -18.32 & 12 & 33 \\
\hline UGC 06036 & 364 & 0.022 & 100 & 0.73 & $\mathrm{Sa}$ & 14.86 & -21.93 & 11 & 38 \\
\hline UGC 06312 & 386 & 0.021 & 49 & 0.64 & $\mathrm{Sab}$ & 10.74 & -21.91 & 13 & 29 \\
\hline UGC 07012 & 486 & 0.010 & 12 & 0.51 & Scd & 0.28 & -19.91 & 14 & 30 \\
\hline UGC 07145 & 500 & 0.022 & 151 & 0.63 & Sbc & 2.26 & -21.14 & 16 & 32 \\
\hline UGC 08107 & 593 & 0.028 & 53 & 0.68 & $\mathrm{Sa}$ & 11.64 & -22.56 & 16 & 33 \\
\hline UGC 08231 & 606 & 0.008 & 73 & 0.66 & $\mathrm{Sd}$ & 0.14 & -19.28 & 19 & 33 \\
\hline UGC 08234 & 607 & 0.027 & 133 & 0.45 & S0 & 13.65 & -22.76 & 8 & 24 \\
\hline UGC 08733 & 657 & 0.008 & 21 & 0.44 & $\mathrm{Sdm}$ & 0.26 & -19.75 & 30 & 40 \\
\hline UGC 08778 & 664 & 0.011 & 116 & 0.70 & $\mathrm{Sb}$ & 1.76 & -20.30 & 15 & 27 \\
\hline UGC 08781 & 665 & 0.025 & 160 & 0.40 & $\mathrm{Sb}$ & 11.38 & -22.37 & 15 & 29 \\
\hline UGC 09067 & 714 & 0.026 & 12 & 0.54 & $\mathrm{Sbc}$ & 3.82 & -21.85 & 14 & 28 \\
\hline UGC 09476 & 769 & 0.011 & 132 & 0.34 & $\mathrm{Sbc}$ & 1.61 & -20.95 & 21 & 40 \\
\hline UGC 09537 & 774 & 0.029 & 140 & 0.79 & $\mathrm{Sb}$ & 16.60 & -22.64 & 20 & 40 \\
\hline UGC 09542 & 775 & 0.018 & 34 & 0.70 & Sc & 2.07 & -20.96 & 21 & 37 \\
\hline UGC 09665 & 783 & 0.009 & 138 & 0.73 & $\mathrm{Sb}$ & 0.99 & -19.99 & 18 & 33 \\
\hline UGC 09873 & 797 & 0.019 & 126 & 0.75 & $\mathrm{Sb}$ & 1.25 & -20.38 & 21 & 33 \\
\hline UGC 09892 & 798 & 0.019 & 101 & 0.69 & $\mathrm{Sbc}$ & 1.98 & -20.71 & 16 & 26 \\
\hline UGC 10097 & 814 & 0.020 & 114 & 0.18 & E5 & 28.71 & -22.73 & 14 & 27 \\
\hline UGC 10123 & 818 & 0.013 & 53 & 0.77 & $\mathrm{Sab}$ & 3.32 & -20.55 & 18 & 31 \\
\hline UGC 10205 & 822 & 0.022 & 133 & 0.38 & SOa & 9.93 & -22.32 & 19 & 35 \\
\hline UGC 10257 & 825 & 0.013 & 162 & 0.78 & $\mathrm{Sbc}$ & 1.21 & -20.47 & 20 & 38 \\
\hline UGC 10297 & 827 & 0.008 & 179 & 0.83 & Sc & 0.29 & -19.11 & 18 & 40 \\
\hline UGC 10331 & 828 & 0.015 & 140 & 0.76 & Sc & 0.77 & -20.43 & 19 & 41 \\
\hline UGC 10337 & 830 & 0.029 & 63 & 0.72 & $\mathrm{Sb}$ & 10.79 & -22.17 & 17 & 26 \\
\hline UGC 10380 & 834 & 0.029 & 108 & 0.79 & $\mathrm{Sb}$ & 10.21 & -21.85 & 12 & 35 \\
\hline UGC 10384 & 837 & 0.017 & 92 & 0.73 & $\mathrm{Sb}$ & 1.87 & -20.73 & 11 & 35 \\
\hline UGC 10388 & 838 & 0.015 & 128 & 0.70 & $\mathrm{Sa}$ & 6.56 & -21.19 & 11 & 28 \\
\hline UGC 10650 & 843 & 0.010 & 22 & 0.78 & Scd & 0.20 & -19.32 & 23 & 43 \\
\hline UGC 10693 & 845 & 0.028 & 103 & 0.37 & E7 & 32.14 & -23.39 & 22 & 31 \\
\hline UGC 10695 & 846 & 0.028 & 110 & 0.35 & E5 & 19.95 & -22.70 & 24 & 27 \\
\hline UGC 10710 & 847 & 0.028 & 147 & 0.65 & $\mathrm{Sb}$ & 9.68 & -22.12 & 20 & 36 \\
\hline UGC 10796 & 852 & 0.010 & 59 & 0.42 & Scd & 0.28 & -19.56 & 20 & 32 \\
\hline UGC 10811 & 854 & 0.029 & 91 & 0.66 & $\mathrm{Sb}$ & 7.48 & -21.92 & 12 & 29 \\
\hline UGC 10905 & 858 & 0.027 & 173 & 0.56 & SOa & 40.46 & -22.92 & 15 & 25 \\
\hline UGC 10972 & 861 & 0.016 & 54 & 0.78 & $\mathrm{Sbc}$ & 2.66 & -21.22 & 24 & 34 \\
\hline UGC 11228 & 865 & 0.019 & 178 & 0.33 & So & 12.39 & -22.10 & 12 & 33 \\
\hline UGC 11649 & 872 & 0.013 & 63 & 0.22 & $\mathrm{Sab}$ & 3.70 & -21.38 & 19 & 32 \\
\hline UGC 11680NED01 & 873 & 0.026 & 57 & 0.46 & $\mathrm{Sb}$ & 12.39 & -22.56 & 16 & 28 \\
\hline UGC 11717 & 877 & 0.021 & 37 & 0.61 & $\mathrm{Sab}$ & 6.95 & -21.84 & 17 & 39 \\
\hline UGC 12054 & 885 & 0.007 & 47 & 0.74 & $\mathrm{Sc}$ & 0.10 & -18.41 & 15 & 33 \\
\hline
\end{tabular}


Table B.1. continued.

\begin{tabular}{lccccccccc}
\hline \hline Galname & ID & $z$ & $\begin{array}{c}\text { PA } \\
(\mathrm{deg})\end{array}$ & $\epsilon$ & Type & $\begin{array}{c}M_{*} \\
\left(10^{10} M_{\odot}\right)\end{array}$ & $\begin{array}{c}M_{r} \\
(\mathrm{mag})\end{array}$ & $\begin{array}{c}R_{\text {eff }} \\
(\operatorname{arcsec})\end{array}$ & $\begin{array}{c}R_{\max } \\
(\operatorname{arcsec})\end{array}$ \\
\hline UGC 12127 & 888 & 0.027 & 0 & 0.11 & $\mathrm{E} 1$ & 23.39 & -23.47 & 36 & 25 \\
UGC 12185 & 890 & 0.022 & 159 & 0.56 & $\mathrm{Sb}$ & 4.68 & -21.56 & 12 & 33 \\
UGC 12274 & 894 & 0.026 & 143 & 0.68 & $\mathrm{Sa}$ & 14.19 & -22.08 & 17 & 27 \\
UGC 12308 & 895 & 0.008 & 118 & 0.79 & $\mathrm{Scd}$ & 0.11 & -18.88 & 27 & 38 \\
UGC 12494 & 905 & 0.014 & 37 & 0.67 & $\mathrm{Sd}$ & 0.28 & -19.67 & 20 & 43 \\
UGC 12518 & 910 & 0.009 & 23 & 0.64 & $\mathrm{Sb}$ & 1.80 & -19.45 & 17 & 34 \\
UGC 12519 & 909 & 0.015 & 157 & 0.70 & $\mathrm{Sc}$ & 1.09 & -20.56 & 21 & 34 \\
UGC 12723 & 926 & 0.018 & 75 & 0.82 & $\mathrm{Sc}$ & 0.76 & -19.77 & 17 & 27 \\
UGC 12810 & 929 & 0.027 & 56 & 0.61 & $\mathrm{Sbc}$ & 5.43 & -22.01 & 20 & 35 \\
UGC 12816 & 930 & 0.018 & 140 & 0.50 & $\mathrm{Sc}$ & 0.66 & -20.63 & 16 & 34 \\
UGC 12857 & 934 & 0.008 & 35 & 0.72 & $\mathrm{Sbc}$ & 0.56 & -19.49 & 19 & 36 \\
UGC 12864 & 935 & 0.016 & 110 & 0.61 & $\mathrm{Sc}$ & 1.13 & -20.69 & 27 & 38 \\
VV488NED02 & 892 & 0.016 & 70 & 0.77 & $\mathrm{Sb}$ & 2.32 & -20.96 & 23 & 33 \\
\hline
\end{tabular}

